\title{
SOLITON RESOLUTION FOR THE DERIVATIVE NONLINEAR SCHRÖDINGER EQUATION
}

\author{
ROBERT JENKINS, JIAQI LIU, PETER A. PERRY, AND CATHERINE SULEM
}

\begin{abstract}
We study the derivative nonlinear Schrödinger equation for generic initial data in a weighted Sobolev space that can support bright solitons (but exclude spectral singularities). Drawing on previous well-posedness results, we give a full description of the long-time behavior of the solutions in the form of a finite sum of localized solitons and a dispersive component. At leading order and in space-time cones, the solution has the form of a multisoliton whose parameters are slightly modified from their initial values by soliton-soliton and soliton-radiation interactions. Our analysis provides an explicit expression for the correction dispersive term. We use the nonlinear steepest descent method of Deift and Zhou [8] revisited by the $\bar{\partial}$-analysis of McLaughlin-Miller [24] and Dieng-McLaughlin [9], and complemented by the recent work of Borghese-Jenkins-McLaughlin [1] on soliton resolution for the focusing nonlinear Schrödinger equation. Our results imply that $N$-soliton solutions of the derivative nonlinear Schrödinger equation are asymptotically stable.
\end{abstract}

\section{Contents}

1. Introduction

1.1. The Inverse Scattering Method

1.2. Soliton Resolution

2. Deformation to a mixed $\bar{\partial}$-Riemann-Hilbert Problem 12

2.1. Conjugation 13

2.2. $\bar{\partial}$-extensions of jump factorization $\quad 15$

3. Decomposition into a $\mathrm{RH}$ model problem and a pure $\bar{\partial}$-problem $\quad 19$

3.1. The outer model: the soliton component 21

3.2. Local model at the stationary phase point 22

3.3. Existence theory for the RH model problem 26

3.4. The remaining $\bar{\partial}$ problem 30

4. Large-time asymptotics for solutions of DNLS 34

4.1. Large-time asymptotic for solution $q$ of (1.3) 34

4.2. Large-time asymptotic for solution $u$ of (1.1) 35

Acknowledgements 37

Appendix A. The Weak Plancherel Formula 37

Appendix B. Solutions of RHP 1.2 for reflectionless scattering data $\quad 39$

References 43

Date: February 20, 2018.

Peter Perry supported in part by NSF Grant DMS-1208778 and by Simons Foundation Research and Travel Grant 359431.

C. Sulem supported in part by NSERC Grant 46179-13. 


\section{INTRODUCTION}

In this paper, we prove soliton resolution for the derivative nonlinear Schrödinger equation (DNLS)

$$
\begin{aligned}
i u_{t}+u_{x x}-i \varepsilon\left(|u|^{2} u\right)_{x} & =0 \\
u(x, t=0) & =u_{0}
\end{aligned}
$$

for initial data in a dense and open subset of the weighted Sobolev space $H^{2,2}(\mathbb{R})$ which contains 0 as well as initial data of arbitrarily large $L^{2}$-norm. Here $\varepsilon= \pm 1$ and $H^{2,2}(\mathbb{R})$ is the completion of $C_{0}^{\infty}(\mathbb{R})$ in the norm

$$
\|u\|_{H^{2,2}}=\left(\left\|(1+(\cdot))^{2} u(\cdot)\right\|_{L^{2}}^{2}+\left\|u^{\prime \prime}\right\|_{L^{2}}^{2}\right)^{1 / 2} .
$$

Our work builds on three previous papers [22, 23, 17] where we respectively considered global well-posedness in the soliton-free sector, large-time asymptotics in the soliton-free sector, and global well-posedness on the dense and open subset described in what follows. We will refer to these as Papers I, II, and III for the remainder of the introduction. A more detailed presentation of the material in Paper III and the current paper may be found in the preprint [17].

Soliton resolution refers to the property that the solution decomposes into the sum of a finite number of separated solitons and a radiative part as $|t| \rightarrow \infty$. The limiting soliton parameters are slightly modulated, due to the soliton-soliton and soliton-radiation interactions. We fully describe the dispersive part which contains two components, one coming from the continuous spectrum and another one from the interaction of the discrete and continuous spectrum.

This decomposition is a central feature in nonlinear wave dynamics and has been the object of many theoretical and numerical studies. It has been established in many perturbative contexts, that is when the initial condition is close to a soliton or a multi-soliton. In non-perturbative cases, this property was proved rigorously for $\mathrm{KdV}[14], \mathrm{mKdV}[7,27]$ and for the focussing NLS equation [1] using the inverse scattering approach. The last result has been conjectured for a long time [30] but rigorously proved only recently. A direct consequence of this result is that $\mathrm{N}$-soliton solutions are asymptotically stable.

The soliton resolution conjecture is at the heart of current studies in nonlinear waves and extends to solutions that blow up in finite time. In the context of non-integrable equations, Tao [28] considered the NLS equation with potential in high dimension $(d \geqslant 11)$ and proved the existence of a global attractor, assuming radial symmetry. A recent work by Duykaerts, Jia, Kenig and Merle [12] concerns the focusing energy critical wave equation for which they prove that any bounded solution asymptotically behaves like a finite sum of modulated solitons, a regular component in the finite time blow-up case or a free radiation in the global case, plus a residue term that vanishes asymptotically in the energy space as time approaches the maximal time of existence (see also $[11,13]$ for other cases, radial and non-radial, in various dimensions).

1.1. The Inverse Scattering Method. In this subsection we briefly review the global well-posedness result of [16] and describe the spectrally defined, dense open subset of initial data $H^{2,2}(\mathbb{R})$ for which we will prove soliton resolution. Equation 
(1a) is gauge-equivalent to the equation

$$
\begin{array}{r}
i q_{t}+q_{x x}+i \varepsilon q^{2} \bar{q}_{x}+\frac{1}{2}|q|^{4} q=0, \\
q(x, t=0)=q_{0}(x)
\end{array}
$$

via the gauge transformation ${ }^{1}$

$$
q(x):=\mathcal{G}(u)(x)=\exp \left(i \varepsilon \int_{x}^{\infty}|u(y)|^{2} d y\right) u(x) .
$$

This nonlinear, invertible mapping is an isometry of $L^{2}(\mathbb{R})$, maps soliton solutions to soliton solutions, and maps dense open sets to dense open sets in weighted Sobolev spaces. Consequently, global well-posedness for (2a) on an open and dense set $U$ in $H^{2,2}(\mathbb{R})$ containing data of arbitrary $L^{2}$-norm implies global well-posedness of (1a) on a subset $\mathcal{G}^{-1}(U)$ of $H^{2,2}(\mathbb{R})$ with the same properties. In [17], global well-posedness is established for (2a).

Our analysis exploits the discovery of Kaup and Newell [18] that (2a) has the Lax representation

$$
\begin{aligned}
& L=-i \lambda \sigma_{3}+Q_{\lambda}-\frac{i}{2} \sigma_{3} Q^{2} \\
& A=2 \lambda L+i \lambda\left(Q_{\lambda}\right)_{x}+\frac{1}{2}\left[Q_{x}, Q\right]+\frac{i}{4} \sigma_{3} Q^{4}
\end{aligned}
$$

where

$$
\sigma_{3}=\left(\begin{array}{cc}
1 & 0 \\
0 & -1
\end{array}\right), \quad Q(x)=\left(\begin{array}{cc}
0 & q(x) \\
\varepsilon q(x) & 0
\end{array}\right), \quad Q_{\lambda}(x)=\left(\begin{array}{cc}
0 & q(x) \\
\varepsilon \lambda \overline{q(x)} & 0
\end{array}\right) .
$$

That is, a smooth function $q(x, t)$ solves (2a) if and only if the operator identity

$$
L_{t}-A_{x}+[L, A]=0
$$

holds for the matrices above with $q=q(x, t)$ (so that both matrices depend on $t$ ).

To exploit the Lax representation, we consider the spectral problem

$$
\Psi_{x}=L \Psi
$$

for $\lambda \in \mathbb{C}$ and $2 \times 2$ matrix-valued solutions $\Psi(x ; \lambda)$.

As shown in [17], (4) defines a map $\mathcal{R}$ from $q \in H^{2,2}(\mathbb{R})$ to spectral data, and has an inverse $\mathcal{I}$ defined by a Riemann-Hilbert problem (Riemann-Hilbert Problem 1.2 below) which recovers the potential $q(x)$. Moreover, the spectral data for a solution $q=q(x, t)$ of (2a) obey a linear law of evolution. Thus the solution $\mathcal{M}$ for the Cauchy problem (2a)-(2b) is given by

$$
\mathcal{M}(t) q_{0}=\left(\mathcal{I} \circ \Phi_{t} \circ \mathcal{R}\right) q_{0}
$$

where $\Phi_{t}$ represents the linear evolution on spectral data. To state the results of Paper III that we will use, we describe the set $U$ and the maps $\mathcal{R}, \Phi_{t}$, and $\mathcal{I}$ in greater detail. For any $q \in H^{2,2}(\mathbb{R})$ and $\lambda \in \mathbb{R}$; there exist unique Jost solutions $\Psi^{ \pm}(x, \lambda)$ of $\Psi_{x}=L \Psi$ with respective asymptotics $\lim _{x \rightarrow \pm \infty} \Psi^{ \pm}(x ; \lambda) e^{i \lambda x \sigma_{3}}=I$,

\footnotetext{
1 In papers I and II, we use a slightly different gauge transformation, namely $\exp \left(-i \varepsilon \int_{-\infty}^{x}|u(y)|^{2} d y\right) u(x)$. Both transformations are clearly equivalent, up to the constant phase factor $\exp \left(i \varepsilon \int_{-\infty}^{\infty}\left|u_{0}(y)\right|^{2} d y\right)$. The current transformation has the advantage of slightly simplifying some formulae in the analysis of long time behavior.
} 
where $I$ denotes the $2 \times 2$ identity matrix. The scattering data are defined via the relation

$$
\Psi^{+}(x ; \lambda)=\Psi^{-}(x ; \lambda) T(\lambda), \quad T(\lambda):=\left(\begin{array}{ll}
\alpha(\lambda) & \beta(\lambda) \\
\breve{\beta}(\lambda) & \breve{\alpha}(\lambda)
\end{array}\right)
$$

where, since $\operatorname{det} \Psi^{ \pm}=1$,

$$
\alpha \breve{\alpha}-\beta \breve{\beta}=1 .
$$

Symmetries of (4) imply that, also

$$
\breve{\alpha}(\lambda)=\overline{\alpha(\lambda)}, \quad \breve{\beta}(\lambda)=\varepsilon \lambda \overline{\beta(\lambda)} .
$$

We introduce the reflection coefficient

$$
\rho(\lambda)=\beta(\lambda) / \alpha(\lambda)
$$

and note that $1-\varepsilon \lambda|\rho(\lambda)|^{2}=\frac{1}{|\alpha(\lambda)|^{2}}>0$. We then define the set

$$
P=\left\{\rho \in H^{2,2}(\mathbb{R}): 1-\varepsilon \lambda|\rho(\lambda)|^{2}>0\right\} .
$$

The function $\breve{\alpha}$ may be computed via the 'Wronskian formula'

$$
\breve{\alpha}(\lambda)=\left|\begin{array}{ll}
\psi_{11}^{-}(x, \lambda) & \psi_{12}^{+}(x, \lambda) \\
\psi_{21}^{-}(x, \lambda) & \psi_{22}^{+}(x, \lambda)
\end{array}\right| ;
$$

a standard analysis of (4) shows that if $q \in L^{1}(\mathbb{R}) \cap L^{2}(\mathbb{R})$, the vector-valued functions $\psi_{(1)}^{-}=\left(\psi_{11}^{-}, \psi_{21}^{-}\right)$and $\psi_{(2)}^{+}=\left(\psi_{12}^{+}, \psi_{22}^{+}\right)$have analytic continuations to $\operatorname{Im} \lambda>0$ for each $x$, and decay exponentially fast respectively as $x \rightarrow \pm \infty$. Thus $\breve{\alpha}$ has an analytic continuation to $\mathbb{C}^{+}$. Zeros $\lambda_{j}$ of $\breve{\alpha}$ are eigenvalues of the spectral problem (4) and signal the presence of square-integrable solutions. We have $\psi_{(1)}^{-}\left(x, \lambda_{j}\right)=B_{j} \psi_{(2)}^{+}\left(x, \lambda_{j}\right)$ for nonzero $B_{j}$ and, if the zero $\lambda_{j}$ is simple, we define the associated norming constant $C_{j}$ as $B_{j} / \breve{\alpha}^{\prime}\left(\lambda_{j}\right)$. If $q_{0} \in H^{2,2}(\mathbb{R})$, zeros of $\breve{\alpha}$ in $\mathbb{C}^{+}$ correspond to bright solitons, as our results on large-time asymptotics show.

Zeros of $\breve{\alpha}$ on $\mathbb{R}$ are known to occur and they correspond to spectral singularities [32]. They are excluded from our analysis in the present paper. Their presence will be addressed in a forthcoming article.

Definition 1.1. We denote by $U$ the subset of $H^{2,2}(\mathbb{R})$ consisting of functions $q$ for which $\breve{\alpha}$ has no zeros on $\mathbb{R}$ and at most finitely many simple zeros in $\mathbb{C}^{+}$.

Clearly $U=\bigcup_{N=0}^{\infty} U_{N}$ where $U_{N}$ consists of those $q$ for which $\breve{\alpha}$ has exactly $N$ zeros in $\mathbb{C}^{+}$. If $N \neq 0$, we denote by $\lambda_{1}, \ldots, \lambda_{N}$ the simple zeros of $\breve{\alpha}$ in $\mathbb{C}^{+}$.

Associated to each $\lambda_{j}$ is a norming constant $C_{j} \in \mathbb{C} \backslash\{0\}:=\mathbb{C}^{\times}$which can be described in terms of the Jost solutions. Thus, the spectral problem (4) associates to each $q \in U_{N}$ the spectral data

$$
\mathcal{D}(q)=\left(\rho,\left\{\left(\lambda_{j}, C_{j}\right)\right\}_{j=1}^{N}\right) \in P \times\left(\mathbb{C}^{+} \times \mathbb{C}^{\times}\right)^{N} .
$$

The map $q \mapsto \mathcal{D}(q)$ is called the direct scattering map. To describe its range, let

$$
V=\bigcup_{N=0}^{\infty} V_{N}, \quad V_{N}=P \times\left(\mathbb{C}^{+} \times \mathbb{C}^{\times}\right)^{N}
$$

For an element $\mathcal{D}$ of $V$, write

$$
\Lambda=\Lambda^{+} \cup \overline{\Lambda^{+}}, \quad \Lambda^{+}=\left\{\lambda_{1}, \ldots, \lambda_{N}\right\}
$$


and define

$$
d_{\Lambda}=\inf _{\lambda, \mu \in \Lambda, \lambda \neq \mu}|\lambda-\mu| .
$$

Note that for any $\lambda_{j} \in \Lambda,\left|\operatorname{Im} \lambda_{j}\right| \geqslant(1 / 2) d_{\Lambda}$. We say that a subset $V^{\prime}$ of $V_{N}$ is bounded if:

(i) $1-\varepsilon \lambda|\rho(\lambda)|^{2} \geqslant c_{1}>0$ for a fixed strictly positive constant $c_{1}$,

(ii) $\sup _{j}\left(\left|C_{j}\right|+\left|\lambda_{j}\right|\right) \leqslant C$ for a fixed strictly positive constant $C$,

(iii) $d_{\Lambda} \geqslant c_{2}>0$ for a fixed strictly positive constant $c_{2}$.

We say that a subset $U^{\prime}$ of $U$ is bounded if the set $\{\mathcal{D}(q): q \in U\}$ is a bounded subset of $V_{N}^{\prime}$ and if, also, $U^{\prime}$ is a bounded subset of $H^{2,2}(\mathbb{R})$.

If $q=q(x, t)$ evolves according to (2a), the corresponding scattering data evolves linearly:

$$
\dot{\rho}(\lambda, t)=-4 i \lambda^{2} \rho(\lambda, t), \quad \dot{\lambda_{j}}=0, \quad \dot{C}_{j}=-4 i \lambda_{j}^{2} C_{j} .
$$

We denote by $\Phi_{t}$ the linear evolution on scattering data $\left\{\rho,\left\{\lambda_{j}, C_{j}\right\}_{j=1}^{N}\right\}$ induced by (12).

Let us introduce the phase function

$$
\theta(x, t, \lambda)=-\left(\frac{x}{t} \lambda+2 \lambda^{2}\right) .
$$

Here and in what follows we write

$$
\operatorname{ad}\left(\sigma_{3}\right) A=\left[\sigma_{3}, A\right]
$$

for a $2 \times 2$ matrix $A$, so that

$$
e^{i \theta \text { ad } \sigma_{3}} A=e^{i \theta \sigma_{3}} A e^{-i \theta \sigma_{3}} .
$$

The (time-dependent) inverse spectral problem is defined by a Riemann-Hilbert problem (RHP) and a reconstruction formula.

Riemann-Hilbert Problem 1.2. Given $x, t \in \mathbb{R}, \rho \in P$ and $\left\{\left(\lambda_{j}, C_{j}\right)\right\}_{j=1}^{N} \subset$ $\mathbb{C}^{+} \times \mathbb{C}^{\times}$, find a matrix-valued function

$$
N(\lambda ; x, t): \mathbb{C} \backslash(\mathbb{R} \cup \Lambda) \rightarrow S L(2, \mathbb{C})
$$

with the following properties:

(i) $N_{22}(\lambda ; x, t)=\overline{N_{11}(\bar{\lambda} ; x, t)}, N_{21}(\lambda ; x, t)=\varepsilon \lambda \overline{N_{12}(\bar{\lambda} ; x, t)}$,

(ii) There exists $p^{*} \in \mathbb{C}$ so that $N(\lambda ; x, t)=\left(\begin{array}{cc}1 & 0 \\ p^{*} & 1\end{array}\right)+\mathcal{O}\left(\frac{1}{\lambda}\right)$ as $|\lambda| \rightarrow \infty$,

(iii) $N$ has continuous boundary values $N_{ \pm}$on $\mathbb{R}$ and

$$
N_{+}(\lambda ; x, t)=N_{-}(\lambda ; x, t) e^{i t \theta \text { ad } \sigma_{3}} v(\lambda), \quad v(\lambda)=\left(\begin{array}{cc}
1-\varepsilon \lambda|\rho(\lambda)|^{2} & \rho(\lambda) \\
-\varepsilon \lambda \overline{\rho(\lambda)} & 1
\end{array}\right)
$$

(iv) $N(\lambda ; x, t)$ has simple poles at each point $p \in \Lambda$ :

$$
\operatorname{Res}_{\lambda=p} N(\lambda ; x, t)=\lim _{\lambda \rightarrow p} N(\lambda ; x, t) e^{i t \theta \operatorname{ad} \sigma_{3}} v(p)
$$

where for each $\lambda_{j} \in \Lambda^{+}$

$$
v\left(\lambda_{j}\right)=\left(\begin{array}{cc}
0 & 0 \\
\lambda_{j} C_{j} & 0
\end{array}\right), \quad v\left(\overline{\lambda_{j}}\right)=\left(\begin{array}{cc}
0 & \varepsilon \overline{C_{j}} \\
0 & 0
\end{array}\right) .
$$


Given the solution $N(\lambda ; x, t)$ of Riemann-Hilbert Problem 1.2, we can recover the solution $q(x, t)$ from the reconstruction formula

$$
q(x, t)=\lim _{\lambda \rightarrow \infty} 2 i \lambda N_{12}(\lambda ; x, t) .
$$

We denote by $\mathcal{I}$ the map from scattering data $\left\{\rho,\left\{\lambda_{j}, C_{j}\right\}\right\}$ to $q(x, 0)$, so that formally the solution operator for $(2 \mathrm{a})$ is given by

$$
\mathcal{M}(t)=\mathcal{I} \circ \Phi_{t} \circ \mathcal{R} .
$$

In Paper III, we proved:

Theorem 1.3. For $\varepsilon= \pm 1$, the set $U$ is open and dense in $H^{2,2}(\mathbb{R})$. Moreover, the direct scattering map

$$
\mathcal{R}: U \rightarrow V
$$

maps bounded subsets of $U_{N}$ into bounded subsets of $V_{N}$ for each $N$, and is uniformly Lipschitz continuous on bounded subsets of $U_{N}$. The inverse scattering map $\mathcal{I}$ has analogous mapping and Lipschitz properties. Finally, the evolution $\mathcal{M}$ defines a solution operator for (2a) in the sense that

(i) $q_{0} \mapsto \mathcal{M}(t) q_{0}$ is locally Lipschitz continuous on $U_{N}$,

(ii) $t \mapsto \mathcal{M}(t) q_{0}$ defines a continuous curve in $H^{2,2}(\mathbb{R})$, and

(iii) For each $q_{0} \in \mathcal{S}(\mathbb{R}), q(x, t)=\mathcal{M}(t) q_{0}$ is a classical solution of $(2 \mathrm{a})$.

As discussed in Paper III, RHP 1.2 is equivalent to a technically somewhat simpler RHP involving row vector-valued functions.

Riemann-Hilbert Problem 1.4. Given $x, t \in \mathbb{R}, \rho \in P$, and $\left\{\left(\lambda_{j}, C_{j}\right)\right\}_{j=1}^{N}$ in $\left(\mathbb{C}^{+} \times \mathbb{C}^{\times}\right)^{N}$, find a row vector-valued function $n(x, t, z): \mathbb{C} \backslash(\mathbb{R} \cup \Lambda) \rightarrow \mathbb{C}^{2}$ with the following properties:

(i) $n(\lambda ; x, t)=\left(\begin{array}{ll}1 & 0\end{array}\right)+\mathcal{O}\left(\frac{1}{\lambda}\right)$ as $|z| \rightarrow \infty$,

(ii) $n$ has continuous boundary values $n_{ \pm}$on $\mathbb{R}$ and

$$
n_{+}(\lambda ; x, t)=n_{-}(\lambda ; x, t) e^{i t \theta \operatorname{ad}\left(\sigma_{3}\right)} v(\lambda), \quad v(\lambda)=\left(\begin{array}{cc}
1-\varepsilon \lambda|\rho(\lambda)|^{2} & \rho(\lambda) \\
-\varepsilon \lambda \overline{\rho(\lambda)} & 1
\end{array}\right),
$$

(iii) For each $\lambda \in \Lambda$,

$$
\operatorname{Res}_{z=\lambda} n(\lambda ; x, t)=\lim _{z \rightarrow \lambda} n(z ; x, t) e^{i t \theta \operatorname{ad}\left(\sigma_{3}\right)} V(\lambda)
$$

where for each $\lambda \in \Lambda^{+}$

$$
v(\lambda)=\left(\begin{array}{cc}
0 & 0 \\
\lambda C_{\lambda} & 0
\end{array}\right), \quad v(\bar{\lambda})=\left(\begin{array}{cc}
0 & \varepsilon \overline{C_{\lambda}} \\
0 & 0
\end{array}\right) .
$$

1.2. Soliton Resolution. To deduce large-time asymptotics and soliton resolution for (1a), we will first obtain large-time asymptotics for (2a) and then compute the asymptotics of the phase in (3) in terms of spectral data. These two results together will yield large-time asymptotics and a modified form of soliton resolution for (1a).

Long-time asymptotic behavior and solution resolution for (2a) are established for initial data $q_{0} \in U$ using the Deift-Zhou method of steepest descent applied to RHP 1.2. Note that the phase function $\theta$ in (13) has a single critical point at $\xi=-x / 4 t$. From the evolution (12) and the symmetry properties of RHP 1.2 , it suffices to solve Riemann-Hilbert Problem 1.4 for the row vector-valued function $n(\lambda ; x, t)=\left(N_{11}(\lambda ; x, t), N_{12}(\lambda ; x, t)\right)$. 
We choose intervals $\left[v_{1}, v_{2}\right]$ of velocities and $\left[x_{1}, x_{2}\right]$ of initial positions and compute the asymptotic behavior of $q(x, t)$ in space-time regions of the form

$$
\mathcal{S}\left(v_{1}, v_{2}, x_{1}, x_{2}\right)=\left\{(x, t): x=x_{0}+v t \text { for } v \in\left[v_{1}, v_{2}\right], x_{0} \in\left[x_{1}, x_{2}\right]\right\} .
$$

The explicit formula for one-soliton solutions given in (95) shows that a soliton associated to eigenvalue $\lambda$ moves with velocity $(-4 \operatorname{Re} \lambda)$.

In what follows, we set

$$
\Lambda(I)=\{\lambda \in \Lambda: \operatorname{Re}(\lambda) \in I\}=\Lambda^{+}(I) \cup \overline{\Lambda^{+}(I)}
$$

and

$$
N(I)=\left|\Lambda^{+}(I)\right|
$$

Solitons in $\Lambda\left(\left[-v_{2} / 4,-v_{1} / 4\right]\right)$ should be 'visible' within $\mathcal{S}\left(v_{1}, v_{2}, x_{1}, x_{2}\right)$, but remaining solitons will move either too slowly or too fast to be seen in the moving window.

To state our result, we need the following notation. For $\xi \in \mathbb{R}$ and $\eta \in\{-1,+1\}$ where $\eta=\operatorname{sgn} t$, let

$$
\begin{aligned}
& I_{\xi, \eta}^{-}=\{\lambda \in \mathbb{C}: \operatorname{Im} \lambda=0, \quad-\infty<\eta \operatorname{Re} \lambda \leqslant \eta \xi\}, \\
& I_{\xi, \eta}^{+}=\{\lambda \in \mathbb{C}: \operatorname{Im} \lambda=0, \quad \eta \xi<\eta \operatorname{Re} \lambda<\infty\} .
\end{aligned}
$$
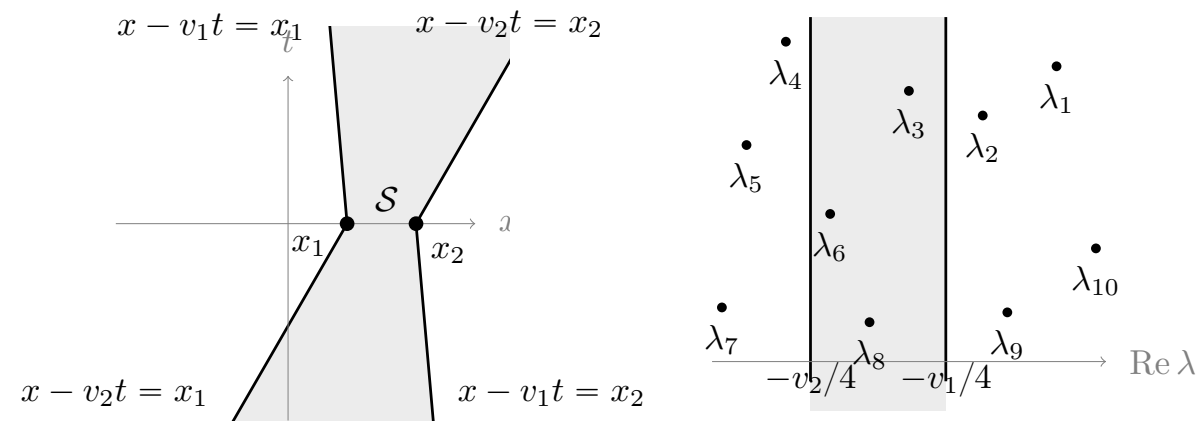

Figure 1. Given initial data $q_{0}(x)$ which generates scattering data $\left\{\rho,\left\{\lambda_{k}, C_{k}\right\}_{k=1}^{N}\right\}$, then, asymptotically as $|t| \rightarrow \infty$ inside the space-time cone $\mathcal{S}\left(v_{1}, v_{2}, x_{1}, x_{2}\right)$ (shaded on left) the solution $q(x, t)$ of (2a) approaches an $N(I)$-soliton $q_{\text {sol }}(x, t)$ corresponding to the discrete spectra in $\Lambda(I)$ (shaded region on right). The connection coefficients $\widehat{C}_{k}$ for $q_{\text {sol }}$ are modulated by the soliton-soliton and soliton-radiation interactions as described in Theorem 1.5.

We will prove:

Theorem 1.5. Suppose that $q_{0} \in U_{N}$ with scattering data $\left(\rho,\left\{\left(\lambda_{k}, C_{k}\right)\right\}_{k=1}^{N}\right)$. Fix $x_{1}, x_{2}, v_{1}, v_{2} \in \mathbb{R}$ with $v_{1}<v_{2}$, let $I=\left[-v_{2} / 4,-v_{1} / 4\right]$ and $\xi=-x /(4 t)$. Denote by $q_{\mathrm{sol}}\left(x, t ; \mathcal{D}_{I}\right)$ the soliton solution of $(2 \mathrm{a})$ with modulating reflectionless scattering data

$$
\mathcal{D}_{I}=\left\{\rho_{I} \equiv 0,\left\{\left(\lambda_{k}, \widehat{C_{k}}\right)\right\}_{\lambda_{k} \in \Lambda\left(I^{+}\right)}\right\}
$$


where

$$
\widehat{C_{k}}=C_{k} \prod_{\operatorname{Re} \lambda_{j} \in I_{\xi, \eta}^{-} \backslash I}\left(\frac{\lambda_{k}-\lambda_{j}}{\lambda_{k}-\overline{\lambda_{j}}}\right)^{2} \exp \left(\frac{i}{\pi} \int_{I_{\xi, \eta}^{-}} \frac{\log \left(1-\varepsilon \lambda|\rho(\lambda)|^{2}\right)}{\lambda-\lambda_{k}} d \lambda\right) .
$$

Then, as $|t| \rightarrow \infty$ in the cone $\mathcal{S}\left(v_{1}, v_{2}, x_{1}, x_{2}\right)$, we have

$$
q(x, t)=q_{\mathrm{sol}}\left(x, t ; \mathcal{D}_{I}\right)+t^{-1 / 2} f(x, t)+\mathcal{O}\left(t^{-3 / 4}\right) .
$$

Here $f(x, t)$ is given by

$$
\begin{aligned}
f(x, t)= & 2^{-1 / 2}\left[A_{12}(\xi, \eta)\left(\mathcal{N}_{11}^{\mathrm{sol}}\right)^{2} e^{i \phi(\xi)}+\varepsilon \xi \overline{A_{12}(\xi, \eta)}\left(\mathcal{N}_{12}^{\mathrm{sol}}\right)^{2} e^{-i \phi(\xi)}\right] \\
\phi(\xi):= & -4 \sum \arg \left(\lambda_{k}-\xi\right) \\
& \operatorname{Re} \lambda_{k} \in I_{\xi, \eta}^{-} \backslash I
\end{aligned}
$$

where $A_{12}(\xi, \eta)$ is given by (47) and $\mathcal{N}^{\text {sol }}:=\mathcal{N}^{\mathrm{sol}}\left(\xi ; x, t \mid \mathcal{D}_{I}\right)$ solves RHP 1.2 with scattering data $\mathcal{D}_{I}$.

Equation (19) expresses soliton resolution in the following sense. First, as described below, the function $q_{\text {sol }}(x, t)$ is generically asymptotic to a superposition of one-soliton solutions. Second, the term at order $t^{-1 / 2}$ represents a dispersive contribution; in the no-soliton case, i.e., if $v_{1}, v_{2}$ are chosen in Theorem 1.5 such that $N(I)=0, \mathcal{N}^{\text {sol }} \equiv I$, and $q_{\text {sol }} \equiv 0$, using (47), the asymptotic behavior of the solution reduces to

$$
q(x, t)=\frac{1}{\sqrt{2|t|}} \frac{\kappa(\xi)}{\xi} e^{i \alpha_{ \pm}(\xi)} e^{i x^{2} /(4 t) \mp \kappa(\xi) \log |8 t|}+\mathcal{O}\left(t^{-3 / 4}\right), \quad t \rightarrow \pm \infty
$$

where

$$
\begin{aligned}
\kappa(s) & =-\frac{1}{2 \pi} \ln \left(1-\varepsilon s|\rho(s)|^{2}\right) \\
\alpha_{ \pm}(\xi) & =\frac{\pi}{4}-\arg (-\varepsilon \xi \overline{\rho(\xi)}) \pm \arg \Gamma(i \kappa(\xi)) \mp 2 \int_{\mp \infty}^{\xi} \log |\xi-\lambda| d \kappa(\lambda)
\end{aligned}
$$

which agrees with the dispersive asymptotics obtained, for example, in [23].

Kitaev and Vartanian computed similar asymptotics in the no-soliton sector [19] and the finite-soliton sector [20], making more stringent assumptions on regularity together with a smallness assumption on the reflection coefficient that we do not require.

Theorem 1.5 implies, as a special case, the asymptotic separation of the solution $q(x, t)$ into a sum of one-soliton solutions whenever the $\lambda_{k} \in \Lambda^{+}$have distinct real parts. If the initial data $q_{0}$ generates scattering data $\left\{\rho,\left\{\left(\lambda_{k}, C_{k}\right)\right\}_{k=1}^{N}\right\}$ with $\lambda_{k}=\eta_{k}+i \tau_{k}$ then applying Theorem 1.5 repeatedly to sets $\mathcal{S}_{k}$ each of which contains a single soliton speed $v_{k}=-4 \eta_{k}$ one finds that the solution of $(2 \mathrm{a})-(2 \mathrm{~b})$ satisfies

$$
q(x, t)=\sum_{k=1}^{N} \mathcal{Q}_{\mathrm{sol}}\left(x, t ; \lambda_{k}, x_{k}^{ \pm}, \varphi_{k}^{ \pm}\right)+\mathcal{O}\left(|t|^{-1 / 2}\right) \quad t \rightarrow \pm \infty
$$

where, setting $u=\operatorname{Re} \lambda$,

$$
\mathcal{Q}_{\text {sol }}\left(x, t ; \lambda, x_{0}, \varphi_{0}\right)=\mathcal{Q}\left(x-x_{0}+4 u t, \lambda\right)
$$




$$
\times \exp i\left\{4|\lambda|^{2} t-2 u(x+4 u t)-\frac{\varepsilon}{4} \int_{-\infty}^{x-x_{0}+4 u t} \mathcal{Q}(\eta, \lambda)^{2} d \eta-\varphi_{0}\right\}
$$

is the form of a general one-soliton solution of (2a). Here $\varphi_{0}, x_{0}$, and $\mathcal{Q}$ are given as in (95). The asymptotic phases are

$$
x_{k}^{ \pm}=\frac{1}{4 \tau_{k}} \log \left|\frac{\lambda_{k} C_{k}^{2}}{4 \tau_{k}^{2}}\right|+\frac{1}{2 \tau_{k}} \sum_{\substack{\lambda_{j} \in \Lambda^{+} \\ \pm\left(\eta_{k}-\eta_{j}\right)>0}} \log \left|\frac{\lambda_{k}-\lambda_{j}}{\lambda_{k}-\overline{\lambda_{j}}}\right| \mp \int_{\eta_{k}}^{\mp \infty} \frac{\kappa(s)}{\left(s-\eta_{k}\right)^{2}+\tau_{k}^{2}} d s
$$

$$
\varphi_{k}^{ \pm}=\arg \left(i \lambda_{k} C_{k}\right)+\sum_{\substack{\lambda_{j} \in \Lambda^{+} \\ \pm\left(\eta_{k}-\eta_{j}\right)>0}} \arg \left(\frac{\lambda_{k}-\lambda_{j}}{\lambda_{k}-\overline{\lambda_{j}}}\right) \pm 2 \int_{\eta_{k}}^{\mp \infty} \frac{\left(s-\eta_{k}\right) \kappa(s)}{\left(s-\eta_{k}\right)^{2}+\tau_{k}^{2}} d s \quad \bmod 2 \pi
$$

so that the total phase shifts of the $k$ th soliton, as it interacts both with the other solitons and the radiation component, are

$$
\begin{aligned}
x_{k}^{+}-x_{k}^{-}= & \frac{1}{2 \tau_{k}} \sum_{j \neq k} \operatorname{sgn}\left(\eta_{k}-\eta_{j}\right) \log \left|\frac{\lambda_{k}-\lambda_{j}}{\lambda_{k}-\overline{\lambda_{j}}}\right|-\int_{-\infty}^{\infty} \frac{\operatorname{sgn}\left(s-\eta_{k}\right) \kappa(s)}{\left(s-\eta_{k}\right)^{2}+\tau_{k}^{2}} d s, \\
\varphi_{k}^{+}-\varphi_{k}^{-}= & {\left[\sum_{j \neq k} \operatorname{sgn}\left(\eta_{k}-\eta_{j}\right) \arg \left(\frac{\lambda_{k}-\lambda_{j}}{\lambda_{k}-\overline{\lambda_{j}}}\right)+2 \int_{-\infty}^{\infty} \frac{\left|s-\eta_{k}\right| \kappa(s)}{\left(s-\eta_{k}\right)^{2}+\tau_{k}^{2}} d s\right] } \\
& \bmod 2 \pi .
\end{aligned}
$$

In the non-generic case in which two or more $\lambda_{j} \in \Lambda^{+}$have the same real part one still observes a form of soliton resolution akin to (21). In this case, the one-solitons in (21) corresponding to spectral values with the same real part coalesce to form higher-order solitons called breathers; these breathers are spatially localized and temporally quasiperiodic when viewed in the moving frame with constant velocity $-4 \operatorname{Re} \lambda$.

To obtain a similar asymptotic formula for (1a), we use the gauge transformation (3) to write

$$
u(x, t)=\left[\mathcal{G}^{-1} \circ \mathcal{M}(t) \circ \mathcal{G}\right]\left(u_{0}\right)(x)
$$

where $\mathcal{M}(t)$ is given by (5). We derive an asymptotic formula for $u(x, t)$ in terms of spectral data for $q_{0}=\mathcal{G}\left(u_{0}\right)$ in Proposition 4.2, which plays a key result and introduces some complications in the asymptotic formulas for small $\xi$. Recall Definition 1.1, the fact that $\mathcal{G}$ maps dense open subsets to dense open sets in $H^{2,2}(\mathbb{R})$, and the space-time region defined in (15). If $u_{0} \in \mathcal{G}^{-1}(U)$, then $q_{0}=\mathcal{G}\left(u_{0}\right)$ has no spectral singularities and the scattering coefficient $\breve{\alpha}$ for $q_{0}$ has at most finitely many zeros. In what follows, set

$$
u_{\mathrm{sol}}\left(x, t ; \mathcal{D}_{I}\right):=\mathcal{G}^{-1}\left(q_{\mathrm{sol}}\left(\cdot, t ; \mathcal{D}_{I}\right)\right)
$$

Theorem 1.6. Suppose that $u_{0} \in \mathcal{G}^{-1}(U)$, let $q_{0}=\mathcal{G}\left(u_{0}\right)$, and

$$
\mathcal{R}\left(q_{0}\right)=\left\{\rho,\left\{\left(\lambda_{k}, C_{k}\right)\right\}_{k=1}^{N}\right\} .
$$

Fix $v_{1}, v_{2}, x_{1}, x_{2}$ as in Theorem 1.5, let $I=\left[-v_{2} / 4,-v_{1} / 4\right], \xi=-x /(4 t)$, and $\eta= \pm 1$ for $\pm t>0$. Fix $M>0$. The solution $u(x, t)$ of (1a) has the following asymptotics as $|t| \rightarrow \infty$ in the cone $\mathcal{S}\left(v_{1}, v_{2}, x_{1}, x_{2}\right)$. 
(i) $\operatorname{For}|\xi| \geqslant M t^{-1 / 8}$,

$$
u(x, t)=u_{\mathrm{sol}}\left(x, t ; \mathcal{D}_{I}\right) e^{i \alpha_{0}(\xi, \eta)}\left[1+t^{-1 / 2} g(x, t)\right]+\mathcal{O}\left(t^{-3 / 4}\right)
$$

(ii) For $|\xi| \leqslant M t^{-1 / 8}$,

$$
u(x, t)=u_{\mathrm{sol}}\left(x, t ; \mathcal{D}_{I}\right) e^{i \alpha_{0}(\xi, \eta)} F(\xi, t, \eta)\left[1+t^{-1 / 2} \widetilde{g}(x, t)\right]+\mathcal{O}\left(t^{-3 / 4}\right) .
$$

In the above formulas, denoting $\mathcal{N}^{\mathrm{sol}}:=\mathcal{N}^{\mathrm{sol}}\left(\xi^{\prime} ; x, t \mid \mathcal{D}_{I}\right)$,

$$
\begin{aligned}
\alpha_{0}(\xi, \eta)= & -2 \int_{I_{\xi, \eta}^{-}} \frac{\kappa(\lambda)}{\lambda} d \lambda+4 \sum_{\operatorname{Re} \lambda_{k} \in I_{\xi, \eta}^{-} \backslash I} \arg \lambda_{k} \\
g(x, t)= & \frac{f(x, t)}{q_{\mathrm{sol}}\left(x, t ; \mathcal{D}_{I}\right)}+2^{1 / 2} \varepsilon \operatorname{Re}\left[A_{12}(\xi, \eta) \mathcal{N}_{11}^{\mathrm{sol}} \overline{\mathcal{N}_{12}^{\mathrm{sol}}} e^{i \phi(\xi)}\right], \\
\tilde{g}(x, t)= & g(x, t) \\
& +(1-G(\xi, t, \eta)) \overline{A_{12}(\xi, \eta)} \exp \left(\begin{array}{c}
4 i \sum_{\operatorname{Re} \lambda_{k} \in I_{\xi, \eta}^{-} \backslash I}^{\arg \lambda_{k}} \\
4
\end{array}\right) \int_{x}^{\infty} u_{\mathrm{sol}}\left(y, t ; \mathcal{D}_{I}\right) d y,
\end{aligned}
$$

where $\phi(\xi)$ is given by (20) and, setting $p=e^{i \pi / 4}\left|8 t \xi^{2}\right|^{1 / 2}$,

$$
F(\xi, t, \eta)=\left[e^{p^{2} / 4} p^{-i \eta \kappa(\xi)} D_{i \eta \kappa(\xi)}(p)\right]^{-2}, \quad G(\xi, t, \eta)=\frac{p D_{i \eta \kappa(\xi)-1}(p)}{D_{i \eta \kappa(\xi)}(p)} .
$$

and $D_{a}(z)$ denote the parabolic cylinder functions whose properties are given in [10].

Remark 1.7. The presence of the phase $e^{i \alpha_{0}(\xi, \eta)}$ in the above formulas represents the mismatch in the phase of $u(x, t)=\mathcal{G}^{-1}(q)$ and $u_{\text {sol }}\left(x, t ; \mathcal{D}_{I}\right)$ as defined in (24) caused by the cumulative interaction of $u_{\text {sol }}\left(y, t ; \mathcal{D}_{I}\right)$ with the radiation and soliton components of the full system which are traveling faster than our chosen reference frame $\mathcal{S}$. Because the velocities are proportional to $-\operatorname{Re} \lambda$ (recall that $v=-4 \operatorname{Re} \lambda_{k}$ for solitons), faster velocities correspond to the part of the spectrum $I_{\xi, \eta}^{-}$which lies to the left (resp. right) of $\xi$ as $t \rightarrow \infty$ (resp. $t \rightarrow-\infty$ ).

An important consequence of our analysis is that it provides a proof of asymptotic stability of N-soliton solutions. Until now, the only known result about stability of DNLS soliton is due to Colin and Ohta [3] who proved orbital stability of 1-soliton solutions in $H^{1}$. Our result gives a detailed description of the long-time behavior of perturbed $N$-solitons in the form of the sum of $N 1$-soliton solutions with parameters close to those of the unperturbed $N$-soliton. It is obtained by combining the Lipschitz continuity of the forward and inverse scattering maps described by Theorems 1.3 and 1.8 of Paper III with the long time result of Theorem 1.5.

Theorem 1.8. Given an $N$-soliton $q_{\mathrm{sol}}\left(x, t ; \mathcal{D}^{\mathrm{sol}}\right)$ solution of $(2 \mathrm{a})$ with initial data in $U_{N}$ such that $\operatorname{Re} \lambda_{k} \neq \operatorname{Re} \lambda_{j}, j \neq k$, with scattering data $\mathcal{D}^{\text {sol }}=\left\{0,\left\{\lambda_{k}^{\text {sol }}, C_{k}^{\text {sol }}\right\}_{k=1}^{N}\right\}$, there exist positive constants $\eta_{0}=\eta_{0}\left(q_{\mathrm{sol}}\right), T=T\left(q_{\mathrm{sol}}\right)$, and $K=K\left(q_{\mathrm{sol}}\right)$ such that any initial data $q_{0} \in H^{2,2}(\mathbb{R})$ with

$$
\eta_{1}:=\left\|q_{0}-q_{\mathrm{sol}}\left(\cdot, 0 ; \mathcal{D}^{\mathrm{sol}}\right)\right\|_{H^{2,2}(\mathbb{R})} \leqslant \eta_{0}
$$


also lies in $U_{N}$, with scattering data $\mathcal{D}=\mathcal{R}\left(q_{0}\right)=\left\{\rho,\left\{\lambda_{k}, C_{k}\right\}_{k=1}^{N}\right\}$ satisfying

$$
\|\rho\|_{H^{2,2}(\mathbb{R})}+\sum_{k=1}^{N}\left|\lambda_{k}-\lambda_{k}^{\mathrm{sol}}\right|+\left|C_{k}-C_{k}^{\mathrm{sol}}\right| \leqslant K \eta_{1}
$$

and the solution of the Cauchy problem (2a)-(2b) asymptotically separates into a sum of $N$ 1-solitons

$$
\sup _{x \in \mathbb{R}}\left|q(x, t)-\sum_{k=1}^{N} \mathcal{Q}_{\text {sol }}\left(x, t ; \lambda_{k}, x_{k}^{ \pm}, \alpha_{k}^{ \pm}\right)\right| \leqslant K \eta_{1}|t|^{-1 / 2}, \quad|t|>T
$$

where the 1-solitons $\mathcal{Q}_{\text {sol }}$ are given by (21)-(23).

To state the corresponding result for (1a) let $u_{\text {sol }}\left(x, t ; \mathcal{D}^{\text {sol }}\right)$ denote the $N$-soliton solution of (1a) such that $\mathcal{R}\left(\mathcal{G}\left(u_{\text {sol }}\right)\right)=\mathcal{D}^{\text {sol }}:=\left\{0,\left\{\left(\lambda_{k}^{\text {sol }}, C_{k}^{\text {sol }}\right)\right\}_{k=1}^{N}\right\}$, i.e.

$$
\mathcal{G}\left(u_{\mathrm{sol}}\left(\cdot, t ; \mathcal{D}^{\mathrm{sol}}\right)\right)=q_{\mathrm{sol}}\left(x, t ; \mathcal{D}^{\mathrm{sol}}\right),
$$

and similarly let

$$
\mathcal{U}_{\mathrm{sol}}\left(x, t ; \lambda, x_{k}, \varphi_{k}\right):=\mathcal{G}^{-1}\left(\mathcal{Q}_{\mathrm{sol}}\left(\cdot, t ; \lambda, x_{k}, \varphi_{k}\right)\right)
$$

be the inverse gauge transformation of the 1-soliton solutions of (2a) defined by (21)-(23). Then applying (1.6) to the previous result gives

Theorem 1.9. Given an $N$-soliton of $(1 \mathrm{a}) u_{\mathrm{sol}}\left(x, t ; \mathcal{D}^{\mathrm{sol}}\right) \in \mathcal{G}^{-1}\left(U_{N}\right)$ as defined above with $\operatorname{Re} \lambda_{k} \neq \operatorname{Re} \lambda_{j}, j \neq k$ there exist positive constants $\eta_{0}=\eta_{0}\left(q_{\mathrm{sol}}\right), T=$ $T\left(q_{\mathrm{sol}}\right)$, and $K=K\left(q_{\mathrm{sol}}\right)$ such that any initial data $u_{0} \in H^{2,2}(\mathbb{R})$ with

$$
\eta_{1}:=\left\|u_{0}-u_{\mathrm{sol}}\left(\cdot, 0 ; \mathcal{D}^{\mathrm{sol}}\right)\right\|_{H^{2,2}(\mathbb{R})} \leqslant \eta_{0}
$$

also lies in $\mathcal{G}^{-1}\left(U_{N}\right)$ with scattering data $\mathcal{D}:=\mathcal{R}\left(\mathcal{G}\left(u_{0}\right)\right)=\left\{\rho,\left\{\lambda_{k}, C_{k}\right\}_{k=1}^{N}\right\}$ satisfying (25). Moreover the solution of the Cauchy problem (1a)-(1b) asymptotically separates into a sum of $N$ 1-solitons

$$
\sup _{x \in \mathbb{R}}\left|u(x, t)-\sum_{k=1}^{N} \mathcal{U}_{\text {sol }}\left(x, t ; \lambda_{k}, x_{k}^{ \pm}, \varphi_{k}^{ \pm}\right) e^{i \alpha_{0}\left(\operatorname{Re} \lambda_{k}, \pm\right)}\right| \leqslant K \eta_{1}|t|^{-1 / 2}, \quad|t|>T
$$

where the phase corrections $\alpha_{0}(\xi, \eta)$ are defined in Theorem 1.6.

Remark 1.10. In both of the above theorems, the condition that the discrete spectral points have distinct real parts is generic are made so that the stability is easier to state. If one considers multi-solitons with spectral points with equal real part (breathers) then the above results still hold for (generic) perturbations which separate the real parts, but the time scale on which the solitons separate will depend upon the particular perturbation.

We close this introduction by sketching the content of this paper. The proofs of Theorems 1.5 and 1.6 are given in Sections 2, 3, and 4, using the steepest descent method of Deift and Zhou [7], the later approach of McLaughlin-Miller [24] and Dieng-McLaughlin [9], and the recent work of Borghese, Jenkins and McLaughlin [1] on the focusing cubic NLS which shows how to treat a problem with discrete as well as continuous spectral data. Following [1], we reduce RHP 1.2 to an 'outer' model which describes the asymptotic behavior of solitons, and an 'inner' model which computes the contributions due to the interactions of solitons and radiation. 
In Section 2, we begin with the row-vector valued function solution of RHP 1.4 and deform it to an RHP on a new contour $\Sigma^{(2)}$ whose jump matrices approach the identity exponentially fast away from the critical point $\xi$ (see Fig. 3). In Section 2.1, we conjugate the row-vector RHP for $n=\left(n_{1}, n_{2}\right)$, by defining a new unknown $n^{(1)}=n \delta^{-\sigma_{3}}$, where $\delta$ is the unique function satisfying Lemma 2.1. The new unknown $n^{(1)}$ is amenable to lensing, i.e., deforming the contour $\mathbb{R}$ about the critical point $\xi$ so that the jump matrix of the deformed Riemann-Hilbert problem approaches the identity exponentially fast away from the critical point $\xi$. In order to deform the contour, we extend the scattering data in the jump matrix of RHP 2.2 into the complex plane to define a new unknown $n^{(2)}$ (see eq. (32)). The new unknown solves a mixed $\bar{\partial}$-Riemann-Hilbert problem, $\bar{\partial}$ /Riemann-Hilbert Problem 1, described in Section 2.2). The extension introduces non-analyticity of $n^{(2)}$ which is solved away at a later step. The solution $n^{(2)}$ coincides with $n^{(1)}$ in the sectors $\Omega_{2}$ and $\Omega_{5}$ (see figure 3 ) and is piecewise analytic in the sectors $\Omega_{1}-\Omega_{6}$ with no jumps across the real axis.

In Section 3, we construct a solution $\mathcal{N}^{\text {RHP }}$ of RHP 3.1, determined by the asymptotic and jump conditions in $\bar{\partial} /$ Riemann-Hilbert Problem 1 . Thus the function

$$
n^{(3)}=n^{(2)}\left(\mathcal{N}^{\mathrm{RHP}}\right)^{-1}
$$

obeys a pure $\bar{\partial}$-problem, $\bar{\partial}$-Problem 3.2. Because the original RHP contains both 'continuous' and 'discrete' data, the solution $\mathcal{N}^{\text {RHP }}$ consists of an 'outer' model for the soliton components (see Section 3.1, RHP 3.5 and Proposition 3.6) and an 'inner' model for the stationary phase point (see Section 3.2, RHP 3.8 and Proposition 3.11). The outer and inner models are used to build a parametrix for RHP 3.1 in Section 3.3. The 'gluing' of parametrices is carried out by solving a small-norm Riemann-Hilbert problem, Riemann-Hilbert Problem 3.14. The $\bar{\partial}$ problem for $n^{(3)}$ is solved in Section 3.4 and is shown to have asymptotic behavior

$$
n^{(3)}(z)=I+\mathcal{O}\left(t^{-3 / 4}\right), \quad I=\left(\begin{array}{ll}
1 & 0 \\
0 & 1
\end{array}\right) \text {. }
$$

Thus, (suppressing the $(x, t)$ dependence for brevity)

$$
n(z)=n^{(3)}(z) \mathcal{N}^{\mathrm{RHP}}(z) \mathcal{R}^{(2)}(z)^{-1} \delta(z)^{\sigma_{3}} .
$$

The leading contribution to $q(x, t)$ in (14) comes from the explicitly computable model factor $\mathcal{N}^{\text {RHP }}$ owing to the asymptotics of $n^{(3)}$, the fact that $\mathcal{R}^{(2)}$ is the identity in sectors $\Omega_{2}$ and $\Omega_{5}$ (and we can take $z \rightarrow \infty$ in either sector), and the diagonal matrix $\delta^{-\sigma_{3}}$ does not change $n_{2}$ at order $\mathcal{O}(1 / z)$.

With the solution of RHP 1.2 in hand, we give the proof of Theorems 1.5 and 1.6 in Sections 4.1 and 4.2, respectively. To prove Theorem 1.6, we establish an asymptotic formula for the phase factor in the gauge transformation (3) in terms of spectral data, Proposition 4.2. This in turn relies on a weak Plancherel formula, Lemma A.2, proved in Appendix A. We construct $N$-soliton solutions $q_{\text {sol }}$ for (2a) in Appendix B.

\section{Deformation to a mixed $\bar{\partial}$-Riemann-Hilbert Problem}

This section is devoted to the two first transformations in the reduction of the original RHP 1.2 to a model that can be solved explicitly and provides the precise behavior of the solution of the DNLS equation for long time up to small terms of 
order $O\left(|t|^{-3 / 4}\right)$. We present the analysis for $t>0$ and $t<0$ simultaneously by introducing the parameter $\eta=\operatorname{sgn}(t)$.

The first step is the conjugation of the solution of RHP 1.2 by a scalar function $\delta$ defined in (26), which is itself the solution of a scalar RHP (Section 2.1). This operation is standard and its effect is described in detail in [8], see also [1] and Paper 2. The second step (Section 2.2) is the deformation of contours from the real axis to the contour $\Sigma^{(2)}$ shown in Figure 3. Our presentation follows Paper 2 with the addition of the treatment of the discrete data associated to the residue conditions [1].

2.1. Conjugation. The long-time asymptotic analysis of RHP 1.2 is determined by the growth and decay of the exponential function $e^{2 i t \theta}$ appearing in both the jump relation (Problem 1.2(iii)) and the residue conditions (Problem 1.2(iv)). Let $\xi=-\frac{x}{2 t}$ be the (unique) critical point of the phase $\theta$ defined in (13). For $|t| \gg 1$, $\left|e^{2 i t \theta}\right| \ll 1$ whenever $\eta \operatorname{Re}(\lambda-\xi)<-c<0$, and $\left|e^{2 i t \theta}\right| \gg 1$ whenever $\eta \operatorname{Re}(\lambda-\xi)>$ $c>0$. RHP 1.2 is formulated from the scattering data in such a way that its solution has identity asymptotics as $x \rightarrow+\infty$ with $t$ fixed. We are interested in the behavior of solutions when $|t| \rightarrow \infty$ with $x / t$ fixed in some interval. It is necessary to renormalize the RHP so that it is well behaved as $t \rightarrow \infty$ with $x / t$ in the interval of interest.
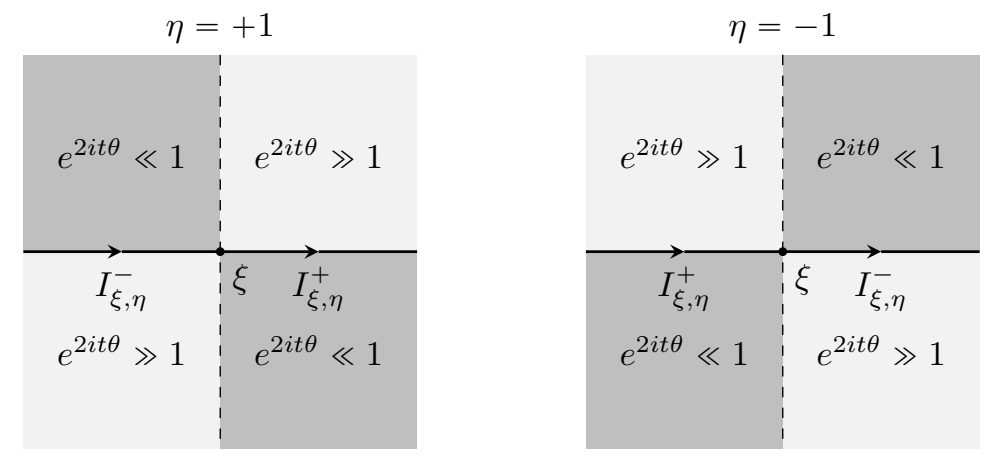

Figure 2. The regions of growth and decay of the exponential factor $e^{2 i t \theta}$ in the $\lambda$-plane for either sign of $\eta=\operatorname{sgn} t$.

Orient the intervals $I_{\xi, \eta}^{ \pm}$from left-to-right, see (18) and Figure 2. Note that if the sign of $t$, and thus $\eta$, is changed with $\xi$ held fixed, the effect is simply to exchange intervals $I_{\xi, \eta}^{ \pm}$. Let

$$
\delta(\lambda)=\delta(\lambda, \xi, \eta):=\exp \left(i \int_{I_{\xi, \eta}^{-}} \frac{\kappa(z)}{z-\lambda} d z\right), \quad \kappa(z)=-\frac{1}{2 \pi} \log \left(1-\varepsilon z|\rho(z)|^{2}\right) .
$$

Lemma 2.1. The function $\delta(\lambda)$ defined by (26) has the following properties:

(i) $\delta$ is meromorphic in $\mathbb{C} \backslash I_{\xi, \eta}^{-}$.

(ii) For $\lambda \in \mathbb{C} \backslash I_{\xi, \eta}^{-}, \delta(\lambda) \overline{\delta(\bar{\lambda})}=1$. Moreover, $e^{-\|\kappa\|_{\infty} / 2} \leqslant|\delta(\lambda)| \leqslant e^{\|\kappa\|_{\infty} / 2}$.

(iii) For $\lambda \in I_{\xi, \eta}^{-}$, $\delta$ 's boundary values $\delta_{ \pm}$, as $\lambda$ approaches the real axis from above and below, satisfy

$$
\delta_{+}(\lambda) / \delta_{-}(\lambda)=1-\varepsilon \lambda|\rho(\lambda)|^{2} .
$$


(iv) As $|\lambda| \rightarrow \infty$ with $|\arg (\eta \lambda)| \neq \pi$,

$$
\delta(\lambda)=1+\frac{\delta_{1}}{\lambda}+\mathcal{O}\left(\lambda^{-2}\right), \quad \delta_{1}=-i \int_{I_{\xi, \eta}^{-}} \kappa(z) d z .
$$

(v) As $\lambda \rightarrow \xi$ along any ray $\xi+e^{i \phi} \mathbb{R}_{+}$with $|\arg (\eta(\lambda-\xi))|<\pi$

$$
\left|\delta(\lambda, \xi, \eta)-\delta_{0}(\xi, \eta)(\eta(\lambda-\xi))^{i \eta \kappa(\xi)}\right| \lesssim_{\rho, \phi}-|\lambda-\xi| \log |\lambda-\xi| .
$$

The implied constant depends on $\rho$ through its $H^{2,2}(\mathbb{R})$-norm and is independent of $\xi$. Here $\delta_{0}(\xi, \eta)=\exp (i \beta(\xi, \xi))$ is a complex unit with

$$
\beta(z, \xi)=-\eta \kappa(\xi) \log (\eta(z-\xi+1))+\int_{I_{\xi, \eta}^{-}} \frac{\kappa(s)-\chi(s) \kappa(\xi)}{s-z} d s,
$$

and $\chi(s)$ is the characteristic function of the interval $\eta \xi-1<\eta s<\eta \xi$. In all of the above formulas, we choose the principal branch of power and logarithm functions.

Proof. Parts $($ i) $-($ iii $)$ are elementary consequences of the definition (26) and the Sokhotski-Plemelj formula. For part $(i v)$, one geometrically expands the factor $(z-\lambda)^{-1}$ for large $\lambda$, and uses the estimate $\|\kappa\|_{L^{1}(\mathbb{R})} \lesssim\|\rho\|_{H^{2,2}(\mathbb{R})}$ to bound the remainder in the integral term for $\lambda$ bounded away from the contour of integration. The proof of part $(v)$ can be found in Appendix A of [23].

We now define a new unknown function $n^{(1)}$ using the function $\delta(\lambda)$ :

$$
n^{(1)}(\lambda)=n(\lambda) \delta(\lambda)^{-\sigma_{3}} .
$$

We claim that $n^{(1)}$ satisfies the following RHP.

Riemann-Hilbert Problem 2.2. Find an analytic row vector-valued function $n^{(1)}: \mathbb{C} \backslash(\mathbb{R} \cup \Lambda) \rightarrow \mathbb{C}^{2}$ with the following properties:

(i) $n^{(1)}(\lambda)=(1,0)+\mathcal{O}\left(\lambda^{-1}\right)$ as $\lambda \rightarrow \infty$.

(ii) For $\lambda \in \mathbb{R}$, the boundary values $n_{ \pm}^{(1)}$ satisfy the jump relation $n_{+}^{(1)}(\lambda)=$ $n_{-}^{(1)}(\lambda) v^{(1)}(\lambda)$ where

$$
v^{(1)}(\lambda)=\left\{\begin{array}{cc}
\left(\begin{array}{cc}
1 & \rho(\lambda) \delta(\lambda)^{2} e^{2 i t \theta} \\
0 & 1
\end{array}\right)\left(\begin{array}{cc}
1 & 0 \\
-\varepsilon \lambda \bar{\rho}(\lambda) \delta(\lambda)^{-2} e^{-2 i t \theta} & 1
\end{array}\right) & \lambda \in I_{\xi, \eta}^{+}, \\
\left(\begin{array}{cc}
1 & 0 \\
\frac{-\varepsilon \lambda \bar{\rho}(\lambda) \delta_{-}(\lambda)^{-2}}{1-\varepsilon \lambda|\rho(\lambda)|^{2}} e^{-2 i t \theta} & 1
\end{array}\right)\left(\begin{array}{cc}
1 & \frac{\rho(\lambda) \delta_{+}(\lambda)^{2}}{1-\varepsilon \lambda|\rho(\lambda)|^{2}} e^{2 i t \theta} \\
0 & 1
\end{array}\right) & \lambda \in I_{\xi, \eta}^{-} .
\end{array}\right.
$$

(iii) $n^{(1)}(\lambda)$ has simple poles at each $p \in \Lambda$ :

$$
\operatorname{Res}_{\lambda=p} n^{(1)}(\lambda)=\lim _{\lambda \rightarrow p} n^{(1)}(\lambda) v^{(1)}(p),
$$

where for each $\lambda_{k} \in \Lambda^{+}$

$$
v^{(1)}\left(\lambda_{k}\right)=\left(\begin{array}{cc}
0 & 0 \\
\lambda_{k} C_{k} \delta\left(\lambda_{k}\right)^{-2} e^{-2 i t \theta} & 0
\end{array}\right), v^{(1)}\left(\bar{\lambda}_{k}\right)=\left(\begin{array}{cc}
0 & \varepsilon \bar{C}_{k} \delta\left(\bar{\lambda}_{k}\right)^{2} e^{2 i t \theta} \\
0 & 0
\end{array}\right) .
$$

Proposition 2.3. Suppose that $n$ satisfies $R H P$ 1.4. Then $n^{(1)}$ defined by (29) satisfies RHP 2.2. 
Proof. The fact that $n^{(1)}$ is analytic in $\mathbb{C} \backslash(\mathbb{R} \cup \Lambda)$, and approaches $(1,0)$ as $\lambda \rightarrow \infty$ follows directly from its definition, Lemma 2.1, and the analytic properties of $n$. The relation $v^{(1)}(\lambda)=\delta_{-}^{\sigma_{3}}(\lambda)\left[e^{i t \theta \operatorname{ad} \sigma_{3}} v(\lambda)\right] \delta_{+}^{\sigma_{3}}(\lambda)$, the standard factorizations of $v$

$$
\begin{aligned}
v & =\left(\begin{array}{ll}
1 & \rho \\
0 & 1
\end{array}\right)\left(\begin{array}{cc}
1 & 0 \\
-\varepsilon \lambda \bar{\rho} & 1
\end{array}\right) \\
& =\left(\begin{array}{cc}
1 & 0 \\
\frac{-\varepsilon \lambda \bar{\rho}}{1-\varepsilon \lambda|\rho|^{2}} & 1
\end{array}\right)\left(\begin{array}{cc}
1-\varepsilon \lambda|\rho|^{2} & 0 \\
0 & \left(1-\varepsilon \lambda|\rho|^{2}\right)^{-1}
\end{array}\right)\left(\begin{array}{cc}
1 & \frac{\rho}{1-\varepsilon \lambda|\rho|^{2}} \\
0 & 1
\end{array}\right),
\end{aligned}
$$

and the jump relation (27) satisfied by $\delta(z)$ on $I_{\xi, \eta}^{-}$allow us to write $v^{(1)}$ as in (30). Concerning the residues (31), as $\delta(\lambda)$ is analytic near each $p \in \Lambda$,

$$
\begin{aligned}
\operatorname{Res}_{\lambda=p} n^{(1)} & =\lim _{\lambda \rightarrow p} n(\lambda) v(p) \delta(p)^{-\sigma_{3}}=\lim _{\lambda \rightarrow p} n^{(1)}(\lambda) \delta(p)^{\sigma_{3}} v(p) \delta(p)^{-\sigma_{3}} \\
& =\lim _{\lambda \rightarrow p} n^{(1)}(\lambda) v^{(1)}(p)
\end{aligned}
$$

with $v^{(1)}$ defined in (31).

2.2. $\bar{\partial}$-extensions of jump factorization. We now introduce a transformation which uses the factorization (30) to deform the jump matrix $v^{(1)}$, replacing it with new jumps along contours in the complex plane which are near identity. Let

$$
\begin{gathered}
\Sigma^{(2)}=\Sigma_{1} \cup \Sigma_{2} \cup \Sigma_{3} \cup \Sigma_{4} \\
\Sigma_{k}=\xi+e^{\frac{i \pi}{4}(2+(2 k-3) \eta)} \mathbb{R}_{+}, \quad k=1,2,3,4,
\end{gathered}
$$

with each ray oriented with increasing (resp. decreasing) real part for $\eta=+1$ (resp. $\eta=-1$ ). The function $e^{2 i t \theta}$ is exponentially increasing along $\Sigma_{1}$ and $\Sigma_{3}$ and decreasing along $\Sigma_{2}$ and $\Sigma_{4}$, while the reverse is true of $e^{-2 i t \theta}$. Let $\Omega_{k}, k=1, \ldots, 6$, denote the six connected components of $\mathbb{C} \backslash\left(\mathbb{R} \bigcup_{k=1}^{4} \Sigma_{k}\right)$, starting with sector $\Omega_{1}$ between $I_{\xi, \eta}^{+}$and $\Sigma_{1}$ and numbered consecutively continuing counterclockwise (resp. clockwise) if $\eta=+1$ (resp. $\eta=-1$ ) as shown in Figure 3.

In order to deform the contour $\mathbb{R}$ to the contour $\Sigma^{(2)}$, we introduce a new unknown $n^{(2)}$

$$
n^{(2)}(\lambda)=n^{(1)}(\lambda) \mathcal{R}^{(2)}(\lambda) .
$$

In each of the sectors $\Omega_{k}, k=1,3,4,6$, which meet the real axis, the condition that $n^{(2)}$ has no jump on the real axis determines the boundary values of $\mathcal{R}^{(2)}$ through the factorization of $v^{(1)}$ in (30). These factorizations involve the reflection coefficient $\rho$ which does not extend analytically to the complex plane. To extend $\mathcal{R}^{(2)}$ off the real axis, we use the method of $[1,4,9]$ which introduces non-analytic extensions. The new unknown $n^{(2)}$ will satisfy a mixed $\bar{\partial}$-RHP. The only condition on the extension is that we have some mild control on $\bar{\partial} \mathcal{R}^{(2)}$ sufficient to ensure that the $\bar{\partial}$-contribution to the long-time asymptotics of $q(x, t)$ is negligible. This is the content of Lemma 2.4 below. We have considerable freedom in choosing the extension. We use this freedom to ensure that: 1) the new jumps on $\Sigma^{(2)}$ match a well known model RHP; 2) in a small neighborhood of each pole in $\Lambda$, $\mathcal{R}^{(2)}(\lambda)=I$-this ensures that the residues are unaffected by the transformation. 

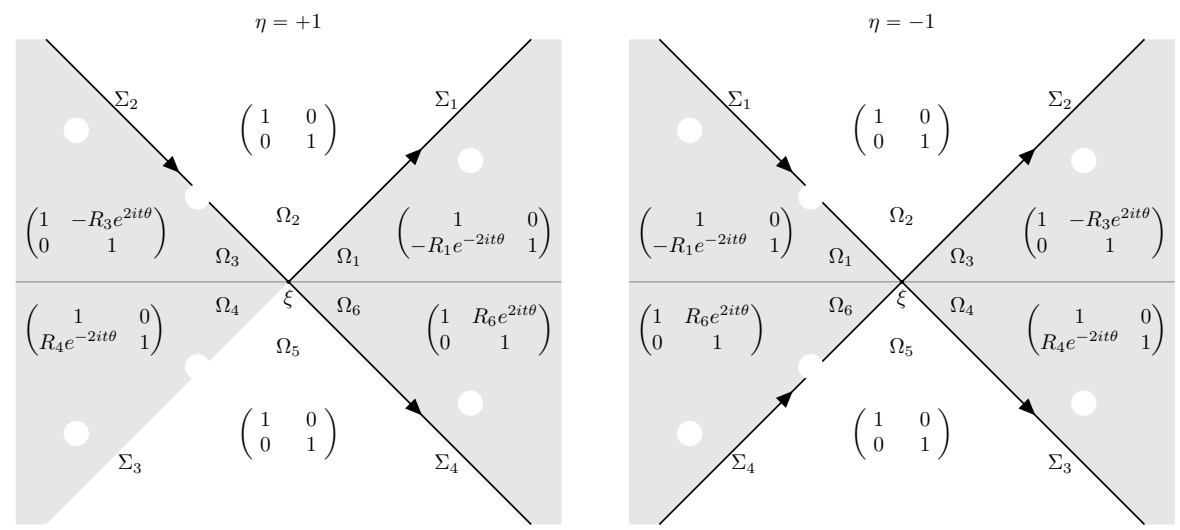

FiguRE 3. Depicted here are the contour $\Sigma^{(2)}=\bigcup_{k=1}^{4} \Sigma_{k}$ and regions $\Omega_{k} k=1, \ldots, 6$ defining the transformation $n^{(2)}=n^{(1)} \mathcal{R}^{(2)}$. The labeling of the regions depends on $\eta$. The non-analytic matrix $\mathcal{R}^{(2)}$ is given in each region $\Omega_{k}$. The support of the $\bar{\partial}$-derivatives, $W^{(2)}=\bar{\partial} \mathcal{R}^{(2)}$ is shaded in gray.

We choose $\mathcal{R}^{(2)}$ as shown in Figure 3 , where the functions $R_{1}, R_{3}, R_{4}, R_{6}$ satisfy

$$
\begin{aligned}
& R_{1}(\lambda)= \begin{cases}-\varepsilon \lambda \overline{\rho(\lambda)} \delta(\lambda)^{-2} & \lambda \in I_{\xi, \eta}^{+} \\
-\varepsilon \xi \overline{\rho(\xi)} \delta_{0}(\xi, \eta)^{-2}(\eta \cdot(\lambda-\xi))^{-2 i \eta \kappa(\xi)}\left(1-\chi_{\Lambda}(\lambda)\right) & \lambda \in \Sigma_{1}\end{cases} \\
& R_{3}(\lambda)= \begin{cases}\frac{\rho(\lambda)}{1-\varepsilon \lambda|\rho(z)|^{2}} \delta_{+}(\lambda)^{2} & \lambda \in I_{\xi, \eta}^{-} \\
\frac{\rho(\xi)}{1-\varepsilon \lambda|\rho(\xi)|^{2}} \delta_{0}(\xi, \eta)^{2}(\eta \cdot(\lambda-\xi))^{2 i \eta \kappa(\xi)}\left(1-\chi_{\Lambda}(\lambda)\right) & \lambda \in \Sigma_{2}\end{cases} \\
& R_{4}(\lambda)= \begin{cases}\frac{-\varepsilon \lambda \overline{\rho(\lambda)}}{1-\varepsilon \lambda|\rho(\lambda)|^{2}} \delta_{-}(\lambda)^{-2} & \lambda \in I_{\xi, \eta}^{-} \\
\frac{-\varepsilon \overline{\rho(\xi)}}{1-\varepsilon \xi|\rho(\xi)|^{2}} \delta_{0}(\xi, \eta)^{-2}(\eta \cdot(\lambda-\xi))^{-2 i \eta \kappa(\xi)}\left(1-\chi_{\Lambda}(\lambda)\right) & \lambda \in \Sigma_{3}\end{cases} \\
& R_{6}(\lambda)= \begin{cases}\rho(\lambda) \delta(\lambda)^{2} \\
\rho(\xi) \delta_{0}(\xi, \eta)^{2}(\eta \cdot(\lambda-\xi))^{2 i \eta \kappa(\xi)}\left(1-\chi_{\Lambda}(\lambda)\right) & \lambda \in I_{\xi, \eta}^{+}\end{cases}
\end{aligned}
$$

Here $\chi_{\wedge}$ is a $C_{0}^{\infty}(\mathbb{C},[0,1])$ cutoff function supported on a neighborhood of each point of the discrete spectrum such that

$$
\chi_{\Lambda}(\lambda)= \begin{cases}1 & \operatorname{dist}(\lambda, \Lambda)<\mathrm{d}_{\Lambda} / 3 \\ 0 & \operatorname{dist}(\lambda, \Lambda)>2 \mathrm{~d}_{\Lambda} / 3\end{cases}
$$

where $\mathrm{d}_{\Lambda}$, defined by (11), is sufficiently small to ensure that the disks of support intersect neither each other nor the real axis.

The following lemma and its proof are almost identical to [9, Proposition 2.1] or [23, Lemma 4.1]. It establishes the existence of the functions $R_{k}$ above and gives estimates that are needed to control the contribution of the solution of the $\bar{\partial}$ problem (Section 3.4) to the large time behavior of $q(x, t)$. To state it, we introduce 
the factors

$$
\begin{array}{ll}
p_{1}(\lambda)=-\varepsilon \overline{\rho(\lambda)}, & p_{3}(\lambda)=\frac{\rho(\lambda)}{1-\varepsilon \lambda|\rho(\lambda)|^{2}}, \\
p_{6}(\lambda)=\rho(\lambda), & p_{4}(\lambda)=\frac{-\varepsilon \overline{\rho(\lambda)}}{1-\varepsilon \lambda|\rho(\lambda)|^{2}} .
\end{array}
$$

We let

$$
P_{k}(\lambda)=\lambda^{m_{k}} p_{k}^{\prime}(\operatorname{Re} \lambda)
$$

where $m_{k}=1$ for $k=1,4$ and $m_{k}=0$ for $k=3,6$.

Lemma 2.4. Suppose that $\rho \in H^{2,2}(\mathbb{R})$ and that $c:=\inf _{\lambda \in \mathbb{R}}\left(1-\varepsilon \lambda|\rho(\lambda)|^{2}\right)>0$ strictly. Then there exist functions $R_{k}$ on $\Omega_{k}, k=1,3,4,6$ satisfying (33), such that

$$
\left|\bar{\partial} R_{k}\right| \lesssim\left\{\begin{array}{lll}
\left|\bar{\partial} \chi_{\Lambda}(\lambda)\right|+\left|P_{k}(\lambda)\right|-\log |\lambda-\xi| & \lambda \in \Omega_{k}, & |\lambda-\xi| \leqslant 1 \\
\left|\bar{\partial} \chi_{\Lambda}(\lambda)\right|+\left|P_{k}(\lambda)\right|+|\lambda-\xi|^{-1} & \lambda \in \Omega_{k}, & |\lambda-\xi|>1
\end{array}\right.
$$

and

$$
\begin{array}{ll}
\bar{\partial} R_{k}(\lambda) \equiv 0 & \text { if } \lambda \in \Omega_{2} \cup \Omega_{5} \text { or } \operatorname{dist}(\lambda, \Lambda) \leqslant \mathrm{d}_{\Lambda} / 3 \\
\bar{\partial} R_{k}(\lambda)=\mathcal{O}(\lambda) & \text { as } \lambda \rightarrow 0 \in \Omega_{1} \cup \Omega_{4}
\end{array}
$$

Here the implied constants are uniform for $\xi \in \mathbb{R}$ and $\rho$ in a fixed bounded subset of $H^{2,2}(\mathbb{R})$ with $1-\varepsilon \lambda|\rho(\lambda)|^{2} \geqslant c>0$ for a fixed constant $c$.

This lemma has the following immediate corollary:

Corollary 2.5. Let $\lambda-\xi=u+i v$ with $u, v \in \mathbb{R}$. Then under the assumptions of Lemma 2.4 for $k=1,3,4,6$, and $\lambda \in \Omega_{k}$, we have

$$
\left|\bar{\partial} \mathcal{R}^{(2)}(\lambda ; \xi)\right| \lesssim \begin{cases}\left(\left|\bar{\partial} \chi_{\Lambda}(\lambda)\right|+\left|P_{k}(\lambda)\right|-\log |z-\xi|\right) e^{-8 t|u||v|} & |\lambda-\xi| \leqslant 1 \\ \left(\left|\bar{\partial} \chi_{\Lambda}(\lambda)\right|+\left|P_{k}(\lambda)\right|\left(1+|\lambda-\xi|^{2}\right)^{-1 / 2}\right) e^{-8 t|u||v|} & |\lambda-\xi|>1\end{cases}
$$

and

$$
\begin{array}{ll}
\bar{\partial} \mathcal{R}^{(2)}(\lambda, \xi) \equiv 0 & \text { if } \lambda \in \Omega_{2} \cup \Omega_{5} \text { or } \operatorname{dist}(\lambda, \Lambda) \leqslant \mathrm{d}_{\Lambda} / 3, \\
\bar{\partial} \mathcal{R}^{(2)}(\lambda, \xi)=\mathcal{O}(\lambda) & \text { as } \lambda \rightarrow 0 \in \Omega_{1} \cup \Omega_{4} .
\end{array}
$$

Here $m_{k}$ is as defined in Lemma 2.4, and all the implied constants are uniform for $\xi \in \mathbb{R}$ and $|t|>1$.

Remark 2.6. The estimates of the $\bar{\partial}$-derivatives at the origin appearing above are used later in the proof of Proposition 3.19 which is needed to compute an asymptotic expansion of the inverse gauge transform $u(x, t)=\mathcal{G}^{-1}(q)(x, t)$.

Proof of Lemma 2.4. We give the construction for $R_{1}$. Define $f_{1}(\lambda)$ on $\Omega_{1}$ by

$$
f_{1}(\lambda)=\xi p_{1}(\xi) \delta_{0}(\xi, \eta)^{-2}(\eta \cdot(\lambda-\xi))^{-2 i \eta \kappa(\xi) \sigma_{3}} \delta(\lambda)^{2}
$$

and let

$$
R_{1}(\lambda)=\left[f_{1}(\lambda)+\left(\lambda p_{1}(\operatorname{Re} \lambda)-f_{1}(\lambda)\right) \eta \cos (2 \phi)\right] \delta(\lambda)^{-2}\left(1-\chi_{\Lambda}(\lambda)\right),
$$


where $\phi=\arg (\lambda-\xi)$. It is easy to see that $R_{1}$, as constructed, satisfies the boundary conditions in (33a) and that $\bar{\partial} R_{1}(\lambda)=0$ for $\operatorname{dist}(\lambda, \Lambda)<\mathrm{d}_{\Lambda} / 3$. Writing $\lambda-\xi=r e^{i \phi}$ we have

$$
\bar{\partial}=\frac{1}{2}\left(\frac{\partial}{\partial \operatorname{Re} \lambda}+i \frac{\partial}{\partial \operatorname{Im} \lambda}\right)=\frac{e^{i \phi}}{2}\left(\frac{\partial}{\partial r}+\frac{i}{r} \frac{\partial}{\partial \phi}\right)
$$

and

$$
\begin{aligned}
\bar{\partial} R_{1}(\lambda) & =-\left[f_{1}(\lambda)+\eta\left(\lambda p_{1}(\operatorname{Re} \lambda)-f_{1}(\lambda)\right) \cos (2 \phi)\right] \delta(\lambda)^{-2} \bar{\partial} \chi_{\Lambda}(\lambda) \\
& +\eta\left[\frac{\lambda}{2} p_{1}^{\prime}(\operatorname{Re} \lambda) \cos (2 \phi)-\frac{i e^{i \phi}}{|z-\xi|}\left(p_{1}(\operatorname{Re} \lambda)-f_{1}(\lambda)\right) \sin (2 \phi)\right] \\
& \times \delta^{-2}(\lambda)\left(1-\chi_{\Lambda}(\lambda)\right) .
\end{aligned}
$$

Clearly, $\bar{\partial} R_{1}(\lambda)=\mathcal{O}(\lambda)$ as $\lambda \rightarrow 0$; it follows from Lemma 2.1, (i) and (v) that

$$
\left|\bar{\partial} R_{1}\right| \lesssim_{\rho} \begin{cases}\left|\bar{\partial} \chi_{\Lambda}(\lambda)\right|+\left|\lambda p_{1}^{\prime}(\operatorname{Re} \lambda)\right|+\log |\lambda-\xi|^{-1}, & |z-\xi| \leqslant 1 \\ \left|\bar{\partial} \chi_{\Lambda}(\lambda)\right|+\left|\lambda p_{1}^{\prime}(\operatorname{Re} \lambda)\right|+|\lambda-\xi|^{-1}, & |z-\xi|>1\end{cases}
$$

where the implied constants depend on $\inf _{\mathbb{R}}\left(1-\varepsilon \lambda|\rho(\lambda)|^{2}\right),\|\rho\|_{H^{2,2}(\mathbb{R})}$, and $\Lambda$. The constructions of $R_{3}, R_{4}$ and $R_{6}$ are similar.

The new unknown $n^{(2)}$ satisfies a mixed $\bar{\partial}$-RHP. We compute the new jumps on $\Sigma^{(2)}$ using the formula

$$
v^{(2)}=n_{-}^{(1)}{ }^{-1} n_{+}^{(1)}=\left(\mathcal{R}_{-}^{(2)}\right)^{-1} v^{(1)} \mathcal{R}_{+}^{(2)}
$$

where the subscripts $+/-$ refer to the left/right side of the contour with respect to its orientation. Away from $\Sigma^{(2)}$, remembering that $n^{(1)}$ is analytic in $\mathbb{C} \backslash(\mathbb{R} \cup \Lambda)$, we have

$$
\bar{\partial} n^{(2)}=n^{(1)} \bar{\partial} \mathcal{R}^{(2)}=n^{(2)}\left(\mathcal{R}^{(2)}\right)^{-1} \bar{\partial} \mathcal{R}^{(2)}=n^{(2)} \bar{\partial} \mathcal{R}^{(2)} .
$$

where the last step follows from the nilpotency of $\bar{\partial} \mathcal{R}^{(2)}$.

$\bar{\partial} /$ Riemann-Hilbert Problem 1. Find a row vector valued-function

$$
n^{(2)}: \mathbb{C} \backslash\left(\Sigma^{(2)} \cup \Lambda\right) \rightarrow \mathbb{C}^{2}
$$

with the following properties:

(i) $n^{(2)}(\lambda)$ has sectionally continuous first partial derivatives in $\mathbb{C} \backslash\left(\Sigma^{(2)} \cup \Lambda\right)$ and continuous boundary values $n_{ \pm}^{(2)}(\lambda)$ on $\Sigma^{(2)}$.

(ii) $n^{(2)}(\lambda)=(1,0)+\mathcal{O}\left(\lambda^{-1}\right)$ as $\lambda \rightarrow \infty$.

(iii) For $\lambda \in \Sigma^{(2)}$, the boundary values satisfy the jump relation

$$
n_{+}^{(2)}(\lambda)=n_{-}^{(2)}(\lambda) v^{(2)}(\lambda)
$$


where

$$
h(\lambda)=\left\{\begin{array}{cc}
v^{(2)}(\lambda)=I+\left(1-\chi_{\Lambda}(\lambda)\right) h(\lambda), \\
\left(\begin{array}{cc}
0 & 0 \\
-\varepsilon \xi \bar{\rho}(\xi) \delta_{0}(\xi, \eta)^{-2}(\eta \cdot(\lambda-\xi))^{-2 i \eta \kappa(\xi)} e^{2 i t \theta} & 0
\end{array}\right) & \lambda \in \Sigma_{1} \\
\left(\begin{array}{cc}
0 & \frac{\rho(\xi) \delta_{0}(\xi, \eta)^{2}}{1-\varepsilon \xi|\rho(\xi)|^{2}}(\eta \cdot(\lambda-\xi))^{2 i \eta \kappa(\xi)} e^{-2 i t \theta} \\
0 & 0
\end{array}\right) & \lambda \in \Sigma_{2} \\
\left(\begin{array}{cc}
0 & 0 \\
\frac{-\varepsilon \xi \bar{\rho}(\xi) \delta_{0}^{-2}(\xi, \eta)}{1-\varepsilon \xi|\rho(\xi)|^{2}}(\eta \cdot(\lambda-\xi))^{-2 i \eta \kappa(\xi)} e^{2 i t \theta} & 0
\end{array}\right) & \lambda \in \Sigma_{3} \\
\left(\begin{array}{cc}
0 & \rho(\xi) \delta_{0}(\xi, \eta)^{2}(\eta \cdot(\lambda-\xi))^{2 i \eta \kappa(\xi)} e^{-2 i t \theta} \\
0 & 0
\end{array}\right) & \lambda \in \Sigma_{4} .
\end{array}\right.
$$

(iv) For $\lambda \in \mathbb{C} \backslash \Sigma^{(2)}$ we have

$$
\bar{\partial} n^{(2)}(\lambda)=n^{(2)}(\lambda) \bar{\partial} \mathcal{R}^{(2)}(\lambda)
$$

where

$$
\bar{\partial} \mathcal{R}^{(2)}(\lambda)=\left\{\begin{array}{cc}
\left(\begin{array}{cc}
0 & 0 \\
-\bar{\partial} R_{1}(\lambda) e^{-2 i t \theta} & 0
\end{array}\right) & \lambda \in \Omega_{1} \\
\left(\begin{array}{cc}
0 & -\bar{\partial} R_{3}(\lambda) e^{2 i t \theta} \\
0 & 0
\end{array}\right) & \lambda \in \Omega_{3} \\
\left(\begin{array}{cc}
0 & 0 \\
\bar{\partial} R_{4}(\lambda) e^{-2 i t \theta} & 0
\end{array}\right) & \lambda \in \Omega_{4} \\
\left(\begin{array}{cc}
0 & \bar{\partial} R_{6}(\lambda) e^{2 i t \theta} \\
0 & 0
\end{array}\right) & \lambda \in \Omega_{6} \\
0 & \text { elsewhere. }
\end{array}\right.
$$

(v) $n^{(2)}(\lambda)$ has simple poles at each point $p \in \Lambda$.

$$
\operatorname{res}_{\lambda=p} n^{(2)}(\lambda)=\lim _{\lambda \rightarrow p} n^{(2)}(\lambda) v^{(1)}(p)
$$

where $v^{(1)}(p)$ is as given in (31).

\section{Decomposition into a RH MOdel Problem AND A PURe $\bar{\partial}$-PROBlem}

The next step in our analysis is to construct the solution $\mathcal{N}^{\mathrm{RHP}}$ of a matrix-valued Riemann-Hilbert problem such that the transformation

$$
n^{(3)}(\lambda)=n^{(2)}(\lambda)\left(\mathcal{N}^{\mathrm{RHP}}(\lambda)\right)^{-1}
$$

results in a pure $\bar{\partial}$-problem, i.e., the new unknown $n^{(3)}$ is continuous; it has no jumps or poles. We arrive at the problem for $\mathcal{N}^{\mathrm{RHP}}$ by essentially ignoring the $\bar{\partial}$ component of $\bar{\partial}$ /Riemann-Hilbert Problem 1. 
Riemann-Hilbert Problem 3.1. Find a $2 \times 2$ matrix valued function $\mathcal{N}^{\mathrm{RHP}}$ : $\mathbb{C} \backslash\left(\Sigma^{(2)} \cup \Lambda\right) \rightarrow S L_{2}(\mathbb{C})$ with the following properties:

(i) $\mathcal{N}^{\mathrm{RHP}}$ satisfies the symmetry relations $\mathcal{N}_{22}^{\mathrm{RHP}}(\lambda)=\overline{\mathcal{N}_{11}^{\mathrm{RHP}}(\bar{\lambda})}$ and $\mathcal{N}_{21}^{\mathrm{RHP}}(\lambda)=$ $\varepsilon \lambda \overline{\mathcal{N}_{12}^{\mathrm{RHP}}(\bar{\lambda})}$.

(ii) $\mathcal{N}^{\mathrm{RHP}}(\lambda)=\left(\begin{array}{ll}1 & 0 \\ \alpha & 1\end{array}\right)+\mathcal{O}\left(\lambda^{-1}\right)$ as $\lambda \rightarrow \infty$, for a constant $\alpha$ determined by the symmetry condition above.

(iii) For $\lambda \in \Sigma^{(2)}$, the boundary values satisfy the jump relation $\mathcal{N}_{+}^{\mathrm{RHP}}(\lambda)=$ $\mathcal{N}_{-}^{\mathrm{RHP}}(\lambda) v^{(2)}(\lambda)$, where $v^{(2)}$ is given by (34).

(iv) $\mathcal{N}^{\overline{R H P}}(\lambda)$ has simple poles at each $p \in \Lambda$ with

$$
\operatorname{res}_{\lambda=p} \mathcal{N}^{\mathrm{RHP}}(\lambda)=\lim _{\lambda \rightarrow p} \mathcal{N}^{\mathrm{RHP}}(\lambda) v^{(\mathrm{RHP})}(p) .
$$

For each $\lambda_{k} \in \Lambda_{+}$

$$
\begin{aligned}
v^{(\mathrm{RHP})}\left(\lambda_{k}\right) & =\left(\begin{array}{cc}
0 & 0 \\
\lambda_{k} C_{k} \delta^{-2}\left(\lambda_{k}\right) e^{-2 i t \theta} & 0
\end{array}\right), \\
v^{(\mathrm{RHP})}\left(\bar{\lambda}_{k}\right) & =\left(\begin{array}{cc}
0 & \varepsilon \overline{C_{k}} \delta^{2}\left(\bar{\lambda}_{k}\right) e^{2 i t \theta} \\
0 & 0
\end{array}\right) .
\end{aligned}
$$

The next step will be solving the following $\bar{\partial}$-problem.

$\bar{\partial}$-Problem 3.2. Given $x, t \in \mathbb{R}$ and $\rho \in H^{2,2}(\mathbb{R})$ with $\inf _{\mathbb{R}}\left(1-\varepsilon \lambda|\rho(\lambda)|^{2}\right)>0$, find a continuous, row vector-valued function $n^{(3)}(\lambda)$ with the following properties:

(i) $n^{(3)}(\lambda) \rightarrow\left(\begin{array}{ll}1 & 0\end{array}\right)$ as $|\lambda| \rightarrow \infty$.

(ii) $\bar{\partial} n^{(3)}(\lambda)=n^{(3)}(\lambda) W^{(3)}(\lambda)$, where

$$
W^{(3)}(\lambda)=\mathcal{N}^{\mathrm{RHP}}(\lambda) \bar{\partial} \mathcal{R}^{(2)}(\lambda)\left(\mathcal{N}^{\mathrm{RHP}}(\lambda)\right)^{-1} .
$$

Lemma 3.3. Suppose that $n^{(2)}$ solves $\bar{\partial}-R H P$ 1. Given a solution $\mathcal{N}^{\mathrm{RHP}}$ of RHP 3.1, the function $n^{(3)}$ defined by (36) satisfies $\bar{\partial}$-Problem 3.2 above.

Proof. Given solutions $n^{(2)}$ and $\mathcal{N}^{\mathrm{RHP}}$ of $\bar{\partial}$-RHP 1 and RHP 3.1 respectively, the normalization condition for $n^{(3)}$ is immediate. As $\mathcal{N}^{\mathrm{RHP}}$ is holomorphic in $\mathbb{C} \backslash \Sigma^{(2)}$, the $\bar{\partial}$-derivative of $n^{(3)}$ satisfies

$$
\bar{\partial} n^{(3)}=\bar{\partial} n^{(2)}\left(\mathcal{N}^{\mathrm{RHP}}\right)^{-1}=\left[n^{(2)} \bar{\partial} \mathcal{R}^{(2)}\right]\left(\mathcal{N}^{\mathrm{RHP}}\right)^{-1}=n^{(3)}\left[\mathcal{N}^{\mathrm{RHP}} \bar{\partial} \mathcal{R}^{(2)}\left(\mathcal{N}^{\mathrm{RHP}}\right)^{-1}\right] .
$$

For $\lambda \in \Sigma^{(2)}$ the computation

$$
\begin{aligned}
n_{+}^{(3)}(\lambda) & =n_{-}^{(3)}(\lambda) \mathcal{N}_{-}^{\mathrm{RHP}}(\lambda) v^{(2)}(\lambda)\left(\mathcal{N}_{+}^{\mathrm{RHP}}(\lambda)\right)^{-1} \\
& =n_{-}^{(3)}(\lambda) \mathcal{N}_{-}^{\mathrm{RHP}}(\lambda) v^{(2)}(\lambda)\left[v^{(2)}(\lambda)^{-1}\left(\mathcal{N}_{-}^{\mathrm{RHP}}(\lambda)\right)^{-1}\right]=n_{-}^{(3)}(\lambda)
\end{aligned}
$$

shows that $n^{(3)}$ has no jumps and is everywhere continuous. Another direct calculation shows that $n^{(3)}$ has removable singularities at each pole in $\Lambda$ : for instance if $p \in \Lambda$ and $v^{(1)}(p)$ is the nilpotent residue matrix in (31) then using (35) and (37) we have the Laurent expansions in $z=\lambda-p$

$$
n^{(2)}(\lambda)=a(p)\left[\frac{v^{(1)}(p)}{z}+I\right]+\mathcal{O}(z) \quad \mathcal{N}^{\mathrm{RHP}}(\lambda)=A(p)\left[\frac{v^{(1)}(p)}{z}+I\right]+\mathcal{O}(z)
$$


where $a(p)$ and $A(p)$ are the constant row vector and matrix in their respective expansions. As $\mathcal{N}^{\mathrm{RHP}} \in S L_{2}(\mathbb{C}),\left(\mathcal{N}^{\mathrm{RHP}}\right)^{-1}=\sigma_{2}\left(\mathcal{N}^{\mathrm{RHP}}\right)^{\top} \sigma_{2}$, and it follows that

$$
\begin{aligned}
n^{(3)}(\lambda) & =n^{(2)}(\lambda) \mathcal{N}^{\mathrm{RHP}}(\lambda)^{-1} \\
& =\left\{a(p)\left[\frac{v^{(1)}(p)}{z}+I\right]+\mathcal{O}(z)\right\}\left\{\left[\frac{-v^{(1)}(p)}{z}+I\right] \sigma_{2} A(p)^{\top} \sigma_{2}+\mathcal{O}(z)\right\} \\
& =\mathcal{O}(1),
\end{aligned}
$$

where the last equality follows from the fact that $v^{(1)}(p)^{2}=0$.

The remainder of this section is dedicated to proving the following proposition

Proposition 3.4. Given $\rho \in H^{2,2}(\mathbb{R})$ with $c:=\inf _{\lambda \in \mathbb{R}}\left(1-\varepsilon \lambda|\rho(\lambda)|^{2}\right)>0$ strictly, then there exists $T>0$ such that for $|t|>T$, there exists a unique solution $\mathcal{N}^{\mathrm{RHP}}(\lambda)$ of RHP 3.1 satisfying

$$
\left\|\mathcal{N}^{\mathrm{RHP}}(\lambda)\right\|_{L^{\infty}\left(\mathbb{C} \backslash B_{\Lambda}\right)} \lesssim 1
$$

where $B_{\Lambda}$ is any open neighborhood of $\Lambda$ and the implied constants are uniform in $x$ and $|t|>T$; they depend on $B_{\Lambda}$ and $\rho$.

To prove the existence of $\mathcal{N}^{\mathrm{RHP}}$, we will first construct two explicit models: one which exactly solves the pure soliton problem obtained by ignoring the jump conditions, and a second which uses parabolic cylinder functions to build a matrix whose jumps exactly match those of $n^{(2)}$ in a neighborhood of the critical point $\xi$. Using our models we prove that $\mathcal{N}^{\mathrm{RHP}}$ exists and extract its behavior for large $t$.

3.1. The outer model: the soliton component. The matrix $\mathcal{N}^{\mathrm{RHP}}$ is meromorphic away from the contour $\Sigma^{(2)}$ on which its boundary values satisfy the jump relation $\mathcal{N}_{+}^{\mathrm{RHP}}(\lambda)=\mathcal{N}_{-}^{\mathrm{RHP}}(\lambda) v^{(2)}(\lambda)$. It is clear from (34) that

$$
\left|v^{(2)}(\lambda)-I\right| \lesssim e^{-2 \sqrt{2} t|\lambda-\xi|^{2}}
$$

where the implied constant depends upon $\mathrm{d}_{\Lambda}$ and $\inf _{\lambda \in \mathbb{R}}\left(1-\varepsilon \lambda|\rho(\lambda)|^{2}\right)$. It follows that outside a fixed neighborhood of $\xi$ we introduce only exponentially small error (in $t$ ) by completely ignoring the jump condition on $\mathcal{N}^{\mathrm{RHP}}$. This results in the following outer model problem

Riemann-Hilbert Problem 3.5. For any fixed $x, t \in \mathbb{R}$, find an analytic function $\mathcal{N}^{\text {out }}:(\mathbb{C} \backslash \Lambda) \rightarrow S L_{2}(\mathbb{C})$ such that

(i) $\mathcal{N}^{\text {out }}$ satisfies the symmetry relations $\mathcal{N}_{22}^{\text {out }}(\lambda)=\overline{\mathcal{N}_{11}^{\text {out }}(\bar{\lambda})}$ and $\mathcal{N}_{21}^{\text {out }}(\lambda)=$ $\varepsilon \lambda \overline{\mathcal{N}_{12}^{\text {out }}(\bar{\lambda})}$

(ii) $\mathcal{N}^{\text {out }}(\lambda)=\left(\begin{array}{ll}1 & 0 \\ \alpha & 1\end{array}\right)+\mathcal{O}\left(\lambda^{-1}\right)$ as $\lambda \rightarrow \infty$, where $\alpha$ is determined via the symmetry condition.

(iii) $\mathcal{N}^{\text {out }}$ has a simple pole at each point in $\Lambda$ satisfying the residue relations in (37) with $\mathcal{N}^{\text {out }}$ replacing $\mathcal{N}^{\mathrm{RHP}}$.

The essential fact we need concerning $\mathcal{N}^{\text {out }}$ is as follows.

Proposition 3.6. The unique solution $\mathcal{N}^{\text {out }}$ of RHP 3.5 is given by

$$
\mathcal{N}^{\text {out }}(\lambda)=\mathcal{N}^{\text {sol }}\left(\lambda \mid \mathcal{D}_{\xi}\right)
$$

where $\mathcal{N}^{\text {sol }}$ is the solution of RHP 1.2 corresponding to the reflectionless scattering data $\mathcal{D}_{\xi}=\left\{\left(\lambda_{k}, \widetilde{C_{k}}\right)\right\}_{k=1}^{N}$ generated by the $N$-soliton solution $q_{\text {sol }}\left(x, t ; \mathcal{D}_{\xi}\right)$ of $(2 \mathrm{a})$. 
Here, $\left\{\lambda_{k}\right\}_{k=1}^{N}$ are the points generated by our original initial data (2b) and the modified connection coefficients are given by

$$
\widetilde{C_{k}}=C_{k} \exp \left(\frac{i}{\pi} \int_{I_{\xi, \eta}^{-}} \log \left(1-\varepsilon z|\rho(z)|^{2}\right) \frac{d z}{z-\lambda_{k}}\right) .
$$

As $\lambda \rightarrow \infty, \mathcal{N}^{\text {out }}$ admits the expansion

$$
\mathcal{N}^{\text {out }}(\lambda)=\left(\begin{array}{cc}
1 & 0 \\
-\frac{\varepsilon \overline{q_{\text {sol }}}\left(x, t ; \mathcal{D}_{\xi}\right)}{2 i} & 1
\end{array}\right)+\frac{\mathcal{N}_{1}^{\text {out }}}{\lambda}+\mathcal{O}\left(\frac{1}{\lambda^{2}}\right),
$$

where

$$
2 i\left(\mathcal{N}_{1}^{\text {out }}\right)_{12}=q_{\text {sol }}\left(x, t ; \mathcal{D}_{\xi}\right) .
$$

Proof. Using formula (26) for $\delta(\lambda)$, RHP 3.5 is identical to Problem B.1 with $\mathcal{D}=\mathcal{D}_{\xi}$ and $\Delta=\varnothing$. Uniqueness follows from Lemma B.3 in Appendix B. The expansion for large $\lambda$ follows from the fact that $\mathcal{N}^{\text {out }}$ is meromorphic, and the given off-diagonal coefficients of the leading and first moment terms follow from (14) and the symmetry condition in RHP 3.5.

Lemma B.3 of Appendix B provides the following useful facts.

Lemma 3.7. Given $\rho \in H^{2,2}(\mathbb{R})$ and $\left\{\left(\lambda_{k}, C_{k}\right)\right\}_{k=1}^{N} \subset \mathbb{C}^{+} \times \mathbb{C}^{\times}$for RHP 1.2, the solution $\mathcal{N}^{\text {out }}$ of $R H P 3.5$ satisfies

$$
\left\|\left(\mathcal{N}^{\text {out }}\right)^{ \pm 1}\right\|_{L^{\infty}\left(\mathbb{C} \backslash \mathcal{B}_{\Lambda}\right)}=\mathcal{O}(1)
$$

where $\mathcal{B}_{\Lambda}$ is any open neighborhood of the points in $\Lambda$, and the implied constant is independent of $(x, t) \in \mathbb{R}^{2}$ and depends on $\rho$ through its $H^{2,2}(\mathbb{R})$ norm.

The dependency of $\mathcal{N}^{\text {out }}$ on $\rho$ appears through the modified connection coefficients defined in Proposition 3.6 above.

3.2. Local model at the stationary phase point. In any neighborhood of the critical point $\lambda=\xi$ the bound (39) does not give a uniformly small estimate of the difference $v^{(2)}-I$ for large times. It follows that our outer model, which replaced $v^{(2)}$ with identity, is not a good approximation of $\mathcal{N}^{\mathrm{RHP}}$ in a neighborhood of $\xi$. We require a new model, $\mathcal{N}^{\mathrm{PC}}$, which is an accurate approximation inside a small-but fixed with respect to $|t|$-neighborhood of $\lambda=\xi$. Let

$$
\mathcal{U}_{\xi}=\left\{\lambda \in \mathbb{C}:|\lambda-\xi| \leqslant \mathrm{d}_{\Lambda} / 3\right\},
$$

where the radius $\mathrm{d}_{\Lambda} / 3$ is chosen such that $\left(1-\chi_{\Lambda}(\lambda)\right) \equiv 1$ for $\lambda \in \mathcal{U}_{\xi}$; this has the effect of making the jump matrix $v^{(2)},(34)$, constant along $\Sigma_{k} \cap \mathcal{U}_{\xi}, k=1, \ldots, 4$.

Define the time-scaled local coordinate

$$
\zeta(\lambda)=|8 t|^{1 / 2}(\lambda-\xi)
$$

Under this change of variables we have the identifications

$$
e^{2 i t \theta}=e^{-i \eta \zeta^{2} / 2} e^{4 i t \xi^{2}}, \quad(\eta \cdot(\lambda-\xi))^{2 i \eta \kappa(\xi)}=(\eta \zeta)^{2 i \eta \kappa(\xi)} e^{-i \eta \kappa(\xi) \log |8 t|} .
$$

Also set,

$$
\begin{gathered}
r_{\xi}:=\rho(\xi) \delta_{0}(\xi, \eta)^{2} e^{-i \eta \kappa(\xi) \log |8 t|} e^{4 i t \xi^{2}}, \quad s_{\xi}:=-\varepsilon \xi \overline{r_{\xi}} \\
1+r_{\xi} s_{\xi}=1-\varepsilon \xi|\rho(\xi)|^{2}
\end{gathered}
$$



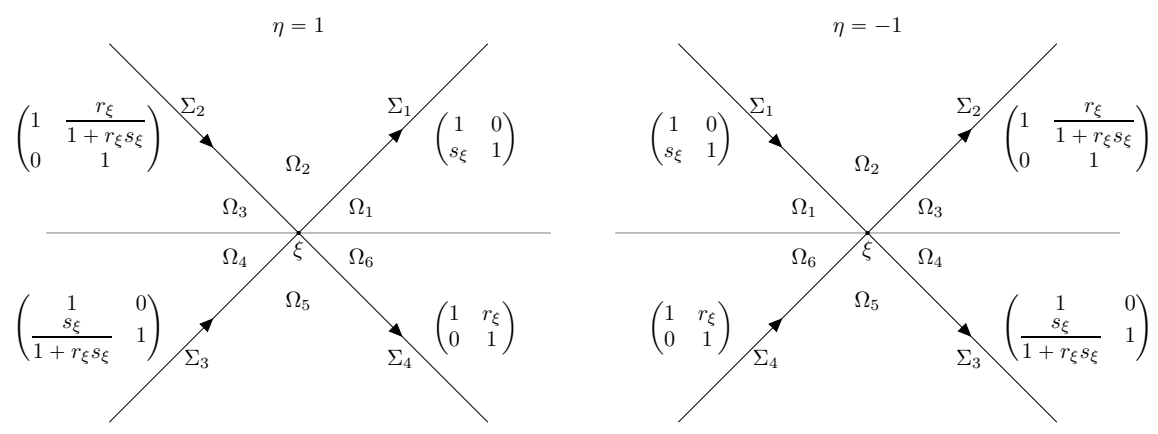

Figure 4. The system of contours for the local model problem near $\lambda=\xi$. The model jumps are $v^{(\mathrm{PC})}=$ $(\eta \zeta)^{i \eta \kappa(\xi) \sigma_{3}} e^{-i \eta \zeta^{2} \sigma_{3} / 4} V(\eta \zeta)^{-i \eta \kappa(\xi) \sigma_{3}} e^{i \eta \zeta^{2} \sigma_{3} / 4}$ where $V$ is given above in terms of the local variable $\zeta$ defined by (41).

Using the notation just introduced, and extending the constant jumps of $\left.v^{(2)}\right|_{\lambda \in \mathcal{U}_{\varepsilon}}$ to infinity along each of the four rays $\Sigma_{k}, k=1, \ldots, 4$, (see Figure 4 ) our local model $\mathcal{N}^{\mathrm{PC}}$ satisfies

Riemann-Hilbert Problem 3.8. Find a $2 \times 2$ matrix-valued function $\mathcal{N}^{\mathrm{PC}}(\lambda)=$ $\mathcal{N}^{\mathrm{PC}}(\lambda ; \xi, \eta)$, analytic in $\mathbb{C} \backslash \Sigma^{(2)}$ with the following properties:

(i) $\mathcal{N}^{\mathrm{PC}}(\lambda ; \xi, \eta)=I+\mathcal{O}\left(\lambda^{-1}\right)$ as $|\lambda| \rightarrow \infty$.

(ii) $\mathcal{N}^{\mathrm{PC}}(\lambda ; \xi, \eta)$ has continuous boundary values $\mathcal{N}_{ \pm}^{\mathrm{PC}}(\lambda ; \xi, \eta)$ on $\Sigma^{(2)}$ which satisfy the jump relation $\mathcal{N}_{+}^{\mathrm{PC}}=\mathcal{N}_{-}^{\mathrm{PC}} v^{(\mathrm{PC})}$, where

$$
v^{(\mathrm{PC})}(\lambda)=\left\{\begin{array}{cc}
\left(\begin{array}{cc}
1 & 0 \\
s_{\xi}(\eta \zeta)^{-2 i \eta \kappa(\xi)} e^{i \eta \zeta^{2} / 2} & 1
\end{array}\right) & \lambda \in \Sigma_{1}, \\
\left(\begin{array}{cc}
1 & \frac{r_{\xi}}{1+r_{\xi} s_{\xi}}(\eta \zeta)^{2 i \eta \kappa(\xi)} e^{-i \eta \zeta^{2} / 2} \\
0 & 1
\end{array}\right) & \lambda \in \Sigma_{2}, \\
\left(\begin{array}{cc}
1 & 0 \\
\frac{s_{\xi}}{1+r_{\xi} s_{\xi}}(\eta \zeta)^{-2 i \eta \kappa(\xi)} e^{i \eta \zeta^{2} / 2} & 1
\end{array}\right) & \lambda \in \Sigma_{3}, \\
\left(\begin{array}{cc}
1 & r_{\xi}(\eta \zeta)^{2 i \eta \kappa(\xi)} e^{-i \eta \zeta^{2} / 2} \\
0 & 1
\end{array}\right) & \lambda \in \Sigma_{4} .
\end{array}\right.
$$

Remark 3.9. RHP 3.8 does not possess the symmetry condition shared by RHP 3.1 and RHP 3.5. This is because it is a local model and will only be used for bounded values of $\lambda$. The normalization is chosen such that the residual error $\mathcal{E}$ defined by (51) below has a near identity jump on the shared boundary between the local and outer models.

Remark 3.10. The jump matrices for RHP 3.8 agree identically with those of RHP 3.1 , and can be solved by special functions, owing to the special choice of $\bar{\partial}$-extension of the matrix factors in (33). By making this choice of extension, one insures that the jumps may be solved exactly 'locally,' i.e., in a neighborhood of the critial point, and produces remainders which can be effectively estimated. Even in the original 
application of the $\bar{\partial}$-method to soliton-free NLS, this method produces an improved remainder estimate for large-time asymptotics (see [9]).

This type of model problem is typical in integrable systems whenever there is a phase function, here $\theta$, which has a quadratic critical point along the real line. The solution in each of these cases is found by a further reduction of RHP 3.8 to a problem with constant jumps (at the price of nontrivial behavior at infinity) whose solution satisfies a differential equation, which can be solved using parabolic cylinder functions, $D_{a}(z)$, whose properties are tabulated in [10, Chapter 12]. The precise details of the construction for DNLS, which differ only slightly from the construction for KdV or NLS (see Deift-Zhou [7] and Its [15]) can be found in [23]; here we give only the necessary details.

Proposition 3.11. Fix $\xi$ and let $\kappa=\kappa(\xi)$ be as given in (26). Then for any choice of constants $r_{\xi}, s_{\xi}$ in (42) such that $1+r_{\xi} s_{\xi}=e^{-2 \pi \kappa} \neq 0$, the solution $\mathcal{N}^{\mathrm{PC}}(\lambda ; \xi, \eta)$ of RHP 3.8 is given by

$$
\begin{aligned}
\mathcal{N}^{\mathrm{PC}}(\lambda ; \xi,+) & =F\left(\zeta(\lambda) ; s_{\xi}, r_{\xi}\right) \\
\mathcal{N}^{\mathrm{PC}}(\lambda ; \xi,-) & =\sigma_{2} F\left(-\zeta(\lambda) ; r_{\xi}, s_{\xi}\right) \sigma_{2}
\end{aligned}
$$

where

$$
\mathcal{P}_{s, r}(\zeta)=\left\{\begin{array}{cccc}
\left(\begin{array}{cc}
1 & 0 \\
s_{\xi} & 1
\end{array}\right) & \arg \zeta \in\left(0, \frac{\pi}{4}\right), & \left(\begin{array}{cc}
1 & \frac{r_{\xi}}{1+r_{\xi} s_{\xi}} \\
0 & 1
\end{array}\right) & \arg \zeta \in\left(\frac{3 \pi}{4}, \pi\right), \\
\left(\begin{array}{cc}
1 & -r_{\xi} \\
0 & 1
\end{array}\right) & \arg \zeta \in\left(-\frac{\pi}{4}, 0\right), & \left(\begin{array}{cc}
1 & 0 \\
\frac{-s_{\xi}}{1+r_{\xi} s_{\xi}} & 1
\end{array}\right) & \arg \zeta \in\left(-\pi,-\frac{3 \pi}{4}\right), \\
I & |\arg \zeta| \in\left(\frac{\pi}{4}, \frac{3 \pi}{4}\right) .
\end{array}\right.
$$

and, writing $\kappa$ for $\kappa(\xi)$,

$$
\Phi_{s, r}(\zeta)=\left(\begin{array}{cc}
e^{-\frac{3 \pi}{4} \kappa} D_{i \kappa}\left(\zeta e^{-3 i \pi / 4}\right) & -i \beta_{12} e^{\frac{\pi}{4}(\kappa-i)} D_{-i \kappa-1}\left(\zeta e^{-\pi i / 4}\right) \\
i \beta_{21} e^{-\frac{3 \pi}{4}(\kappa+i)} D_{i \kappa-1}\left(\zeta e^{-3 i \pi / 4}\right) & e^{\pi \kappa / 4} D_{-i \kappa}\left(\zeta e^{-i \pi / 4}\right)
\end{array}\right)
$$

for $\operatorname{Im}(\zeta)>0$, and for $\operatorname{Im}(\zeta)<0$

$$
\Phi_{s, r}(\zeta)=\left(\begin{array}{cc}
e^{\pi \kappa / 4} D_{i \kappa}\left(\zeta e^{\pi i / 4}\right) & -i \beta_{12} e^{-\frac{3 \pi}{4}(\kappa-i)} D_{-i \kappa-1}\left(\zeta e^{3 i \pi / 4}\right) \\
i \beta_{21} e^{\frac{\pi}{4}(\kappa+i)} D_{i \kappa-1}\left(\zeta e^{\pi i / 4}\right) & e^{-3 \pi \kappa / 4} D_{-i \kappa}\left(\zeta e^{3 i \pi / 4}\right)
\end{array}\right) .
$$

Here

$$
\beta_{12}:=\beta_{12}(s, r)=\frac{\sqrt{2 \pi} e^{-\pi \kappa / 2} e^{i \pi / 4}}{s \Gamma(-i \kappa)} \quad \beta_{21}:=\beta_{21}(s, r)=\frac{-\sqrt{2 \pi} e^{-\pi \kappa / 2} e^{-i \pi / 4}}{r \Gamma(i \kappa)} .
$$

As $\zeta \rightarrow \infty$

$$
F(\zeta ; s, r)=I+\frac{1}{\zeta}\left(\begin{array}{cc}
0 & -i \beta_{12}(s, r) \\
i \beta_{21}(s, r) & 0
\end{array}\right)+\mathcal{O}\left(\zeta^{-2}\right) .
$$

The essential property of $\mathcal{N}^{\mathrm{PC}}$ that we will need later is the asymptotic expansion for large $\zeta$. Using (44), we have 


$$
\mathcal{N}^{\mathrm{PC}}(\lambda ; \xi, \eta)=I+\frac{|8 t|^{-1 / 2}}{\lambda-\xi} A(\xi, \eta)+\mathcal{O}\left(t^{-1}\right), \quad \lambda \in \partial \mathcal{U}_{\xi}
$$

where

$$
A(\xi, \eta)=\left(\begin{array}{cc}
0 & -i A_{12}(\xi, \eta) \\
i A_{21}(\xi, \eta) & 0
\end{array}\right)
$$

with

$$
\begin{aligned}
& A_{12}(\xi,+)=\beta_{12}\left(s_{\xi}, r_{\xi}\right), \quad A_{21}(\xi,+)=\beta_{21}\left(s_{\xi}, r_{\xi}\right) \\
& A_{12}(\xi,-)=-\beta_{21}\left(r_{\xi}, s_{\xi}\right), \quad A_{21}(\xi,-)=-\beta_{12}\left(r_{\xi}, s_{\xi}\right)
\end{aligned}
$$

satisfies

$$
\left|A_{12}(\xi, \eta)\right|^{2}=\frac{\kappa(\xi)}{\xi} \quad A_{21}(\xi, \eta)=\varepsilon \xi \overline{A_{12}(\xi, \eta)}
$$

$$
\begin{aligned}
\arg A_{12}(\xi,+) & =\frac{\pi}{4}+\arg \Gamma(i \kappa(\xi))-\arg (-\varepsilon \xi \overline{\rho(\xi)}) \\
+ & \frac{1}{\pi} \int_{-\infty}^{\xi} \log |\xi-\lambda| \mathrm{d}_{\lambda} \log \left(1-\varepsilon \lambda|\rho(\lambda)|^{2}\right)-\kappa(\xi) \log |8 t|+4 t \xi^{2} \\
\arg A_{12}(\xi,-) & =\frac{\pi}{4}-\arg \Gamma(i \kappa(\xi))-\arg (-\varepsilon \overline{\xi(\xi)}) \\
& +\frac{1}{\pi} \int_{\xi}^{\infty} \log |\xi-\lambda| \mathrm{d}_{\lambda} \log \left(1-\varepsilon \lambda|\rho(\lambda)|^{2}\right)+\kappa(\xi) \log |8 t|+4 t \xi^{2} .
\end{aligned}
$$

For $\eta=+1$, the first line of (46) and (48) are proved in [23]; the results for $\eta=-1$ then follow from (44). The second equality in (48) is a consequence of the fact that $\beta_{12} \beta_{21}=\kappa$. Equations (49) follow simply from (45) and (42) where we use (2.1) and integration by parts to express the integral terms.

Later, we will compute an asymptotic expansion for the solution $u(x, t)$ of (1a)(1b) via the inverse gauge transformation $u(x, t)=\mathcal{G}^{-1}(q)(x, t)$. This can by computed in terms of $1 /\left[n_{11}(0 ; x, t)\right]^{2}$, the solution of RHP 1.4 (cf. Proposition 4.2 below and (83) in particular). When $\xi$ is near zero (specifically, when $0 \in \mathcal{U}_{\xi}$ ) this computation involves the value of the model problem at $\lambda=0$, which from (41) corresponds to $\zeta(0)=-|8 t|^{1 / 2} \xi$ in the model plane. Note that, though (44) is piecewise defined across the real axis, $\mathcal{N}^{\mathrm{PC}}$ does not have a jump across the real axis (cf. (43)); in the formulas below we have chosen the components of $\Phi_{s, r}$ for which right multiplication by $\mathcal{P}_{s, r}(\zeta)$ has no effect. The first column $\mathcal{N}_{1}^{\mathrm{PC}}$ of $\mathcal{N}^{\mathrm{PC}}$ at $\lambda=0$ is given by:

$$
\begin{aligned}
& \mathcal{N}_{1}^{\mathrm{PC}}(0 ; \xi, \eta)=e^{\frac{\pi \kappa(\xi)}{4}} e^{2 i t \xi^{2}-\frac{i}{2} \eta \kappa(\xi) \log \left|8 t \xi^{2}\right|}\left(\begin{array}{cc}
1 & 0 \\
0 & -e^{\frac{i \eta \pi}{4}} \operatorname{sgn}(\xi)
\end{array}\right) \\
& \times\left[\begin{array}{c}
D_{i \eta \kappa(\xi)}\left(e^{\left.\left.\frac{i \eta \pi}{4}\left|8 t \xi^{2}\right|\right|^{1 / 2}\right)}\right. \\
i A_{21}(\xi, \eta) D_{i \eta \kappa(\xi)-1}\left(e^{\frac{i \eta \pi}{4}}\left|8 t \xi^{2}\right|^{1 / 2}\right)
\end{array}\right]
\end{aligned}
$$

Lemma 3.12. Let $c_{1}, c_{2}, c_{3}$ be strictly positive constants, and suppose that $\rho \in$ $H^{2,2}(\mathbb{R})$ with $\|\rho\|_{H^{2,2}(\mathbb{R})} \leqslant c_{1}$, $\inf _{\lambda \in \mathbb{R}}\left(1-\varepsilon \lambda|\rho(\lambda)|^{2}\right) \geqslant c_{2}$, and $|\xi|<c_{3}$. Then as $|t| \rightarrow \infty$,

$$
\left|\mathcal{N}_{21}^{\mathrm{PC}}(0 ; \xi, \eta)\right| \lesssim t^{-1 / 2}
$$


where the implied constant is independent of $\xi$ and $\rho$.

Proof. From (50) and (48) we have, setting $p:=e^{\frac{i \eta \pi}{4}}\left|8 t \xi^{2}\right|^{1 / 2}$,

$$
\left|\mathcal{N}_{21}^{\mathrm{PC}}(0 ; \xi, \eta)\right|=e^{\frac{\pi \kappa(\xi)}{4}}\left|A_{21}(\xi, \eta) D_{i \eta \kappa(\xi)-1}(p)\right|=\left|\frac{\kappa(\xi)}{8 t \xi}\right|^{1 / 2}\left|e^{\frac{\pi \kappa(\xi)}{4}} p D_{i \eta \kappa(\xi)-1}(p)\right|
$$

Since $\kappa(\xi) / \xi \rightarrow \frac{\varepsilon}{2 \pi}|\rho(0)|^{2}$ as $\xi \rightarrow 0$, it is sufficient to show that the last factor is bounded in $p \geqslant 0$. For finite $p$ this is trivial, and for large $p$, the asymptotic expansion of $D_{\nu}(z)$ [10, Eq. 12.9.1] gives

$$
\left|e^{\frac{\pi \kappa(\xi)}{4}} p D_{i \eta \kappa(\xi)-1}(p)\right|=\left|e^{-i p^{2} / 4} p^{i \eta \kappa(\xi)}\left[1+\mathcal{O}\left(p^{-2}\right)\right]\right|=1+\mathcal{O}\left(p^{-2}\right) .
$$

We also need the following boundedness property:

Lemma 3.13 (see [23, Appendix D]). Let $c_{1}$ and $c_{2}$ be strictly positive constants, and suppose that $\rho \in H^{2,2}(\mathbb{R})$ with $\|\rho\|_{H^{2,2}(\mathbb{R})} \leqslant c_{1}$ and $\inf _{\lambda \in \mathbb{R}}\left(1-\varepsilon \lambda|\rho(\lambda)|^{2}\right) \geqslant c_{2}$. Then,

$$
\left\|\mathcal{N}^{\mathrm{PC}}(\cdot ; \xi, \eta)\right\|_{\infty} \lesssim 1 \quad\left\|\mathcal{N}^{\mathrm{PC}}(\cdot ; \xi, \eta)^{-1}\right\|_{\infty} \lesssim 1,
$$

where the implied constants are uniform in $\xi$ and $|t|>1$ and depends only on $c_{1}$ and $c_{2}$.

3.3. Existence theory for the RH model problem. In this section, we prove that the solution $\mathcal{N}^{\mathrm{RHP}}$ of our model problem, RHP 3.1 exists, by constructing it from the outer and local models introduced previously. We will show that for large times, the error solves a small norm Riemann-Hilbert problem which we can expand asymptotically.

Write the solution $\mathcal{N}^{\mathrm{RHP}}$ of RHP 3.1 in the form

$$
\mathcal{N}^{\mathrm{RHP}}(\lambda)= \begin{cases}\mathcal{E}(\lambda) \mathcal{N}^{\text {out }}(\lambda) & |\lambda-\xi| \notin \mathcal{U}_{\xi} \\ \mathcal{E}(\lambda) \mathcal{N}^{\text {out }}(\lambda) \mathcal{N}^{\mathrm{PC}}(\lambda) & |\lambda-\xi| \in \mathcal{U}_{\xi}\end{cases}
$$

where $\mathcal{U}_{\xi}$ is defined in (40), $\mathcal{N}^{\text {out }}$, the solution of RHP 3.5, and $\mathcal{N}^{\mathrm{PC}}$, the solution of RHP 3.8, are both bounded functions of $(x, t)$ having determinant equal to 1 . This relation implicitly defines a transformation to a new unknown $\mathcal{E}$ which satisfies a new $\mathrm{RH}$ problem. In order to state it let

$$
\Sigma^{(\mathcal{E})}=\partial \mathcal{U}_{\xi} \cup\left(\Sigma_{2} \backslash \mathcal{U}_{\xi}\right)
$$

where the circle $\partial \mathcal{U}_{\xi}$ is oriented counterclockwise.

Riemann-Hilbert Problem 3.14. Find a $2 \times 2$ matrix value function $\mathcal{E}$ analytic in $\mathbb{C} \backslash \Sigma^{(\mathcal{E})}$ with the following properties:

(i) For $\lambda \in \mathbb{C} \backslash \mathcal{U}_{\xi}, \mathcal{E}$ satisfies the symmetry relations $\mathcal{E}_{22}(\lambda)=\overline{\mathcal{E}_{11}(\bar{\lambda})}$ and $\mathcal{E}_{21}(\lambda)=\varepsilon \lambda \overline{\mathcal{E}_{12}(\bar{\lambda})}$

(ii) $\mathcal{E}(\lambda)=\left(\begin{array}{cc}1 & 0 \\ \bar{q}_{\mathcal{E}} & 1\end{array}\right)+\mathcal{O}\left(\lambda^{-1}\right)$ as $|\lambda| \rightarrow \infty$, for a constant $\bar{q}_{\mathcal{E}}$ determined by the symmetry condition above.

(iii) For $\lambda \in \Sigma^{(\mathcal{E})}$, the boundary values $\mathcal{E}_{ \pm}$satisfy the jump relation $\mathcal{E}_{+}(\lambda)=$ $\mathcal{E}_{-}(\lambda) v^{(\mathcal{E})}(\lambda)$ where

$$
v^{(\mathcal{E})}(\lambda)= \begin{cases}\mathcal{N}^{\text {out }}(\lambda) v^{(2)}(\lambda) \mathcal{N}^{\text {out }}(\lambda)^{-1} & \lambda \in \Sigma_{2} \backslash \mathcal{U}_{\xi} \\ \mathcal{N}^{\text {out }}(\lambda) \mathcal{N}^{\text {PC }}(\lambda)^{-1} \mathcal{N}^{\text {out }}(\lambda)^{-1} & \lambda \in \partial \mathcal{U}_{\xi} .\end{cases}
$$


The jump matrix $v^{(\mathcal{E})}$ is uniformly near identity for large times; it follows from (39), (46) and Lemma 3.7, that

$$
\left|v^{(\mathcal{E})}(\lambda)-I\right| \lesssim \begin{cases}|t|^{-1 / 2} & \lambda \in \partial \mathcal{U}_{\xi} \\ e^{-2 \sqrt{2}\left|t(\lambda-\xi)^{2}\right|} & \lambda \in \Sigma_{2} \backslash \mathcal{U}_{\xi}\end{cases}
$$

and

$$
\left\|v^{(\mathcal{E})}-I\right\|_{L^{2, k}(\mathbb{R}) \cap L^{\infty}(\mathbb{R})} \lesssim|t|^{-1 / 2}, \quad k \in \mathbb{N}
$$

There is a well known existence and uniqueness theorem for RHPs with near identity jump matrices $[6,29,31]$. Let $C_{\mathcal{E}}$ denote the Cauchy integral operator

$$
C_{\mathcal{E}} f=C^{-}\left(f\left(v^{(\mathcal{E})}-I\right)\right)
$$

where $C^{-}$is the usual Cauchy projection operator on $\Sigma^{(\mathcal{E})}$ :

$$
C^{-} f(\lambda)=\lim _{\lambda \rightarrow \Sigma_{-}^{(\mathcal{E})}} \frac{1}{2 \pi i} \int_{\Sigma^{(\mathcal{E})}} \frac{f(z)}{z-\lambda} d z .
$$

The essential fact needed for the small-norm theory is that $C_{\mathcal{E}}$ is a small norm operator,

$$
\left\|C_{\mathcal{E}}\right\|_{L^{2}\left(\Sigma^{(\mathcal{E})}\right) \rightarrow L^{2}\left(\Sigma^{(\mathcal{E})}\right)}=\mathcal{O}\left(\left\|v^{(\mathcal{E})}-I\right\|_{\infty}\right)=\mathcal{O}\left(|t|^{-1 / 2}\right)
$$

Lemma 3.15. Suppose that $\rho \in H^{2,2}(\mathbb{R})$ and $c:=\inf _{\lambda \in \mathbb{R}}\left(1-\lambda|\rho(\lambda)|^{2}\right)>0$ strictly. Then, for sufficiently large times $|t|>0$, there exists a unique solution $\mathcal{E}(\lambda ; x, t)$ of RHP 3.14 with the property that

$$
\left\|\mathcal{E}-\left(\begin{array}{cc}
1 & 0 \\
\bar{q}_{\mathcal{E}} & 1
\end{array}\right)\right\|_{L^{\infty}(\mathbb{C})} \lesssim|t|^{-1 / 2}
$$

Moreover, as $\lambda \rightarrow \infty$

$$
\mathcal{E}(\lambda)=\left(\begin{array}{cc}
1 & 0 \\
\bar{q}_{\mathcal{E}} & 1
\end{array}\right)+\lambda^{-1} \mathcal{E}_{1}+\mathcal{O}\left(\lambda^{-2}\right)
$$

where $q_{\mathcal{E}}:=\varepsilon\left(\mathcal{E}_{1}\right)_{12}$ and

$$
2 i\left(\mathcal{E}_{1}\right)_{12}=\frac{1}{|2 t|^{1 / 2}}\left[A_{12}(\xi, \eta) \mathcal{N}_{11}^{\text {out }}(\xi)^{2}+A_{21}(\xi, \eta) \mathcal{N}_{12}^{\text {out }}(\xi)^{2}\right]+\mathcal{O}\left(|t|^{-1}\right),
$$

Here, $\mathcal{N}^{\text {out }}$ is the solution of $R H P 3.5$ described in Lemma 3.6 while $A_{12}$ and $A_{21}$ are given by (47)-(49).

Proof. Due to the nonstandard normalization we will construct the solution $\mathcal{E}$ rowby-row. We begin by considering the first row, which we denote $\mathbf{e}_{1}=\left(\begin{array}{ll}\mathcal{E}_{11} & \mathcal{E}_{12}\end{array}\right)$, which is canonically normalized.

By standard results in the theory of Cauchy integral operators [8], $\mathbf{e}_{1}$ must satisfy

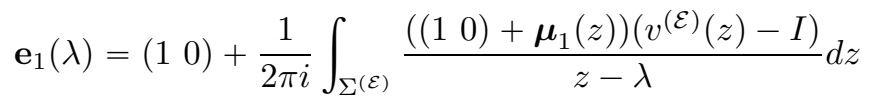

where $\boldsymbol{\mu}_{1} \in L^{2}\left(\Sigma^{(\mathcal{E})}\right)$ is the unique row vector solution of

$$
\left(1-C_{\mathcal{E}}\right) \boldsymbol{\mu}_{1}=C_{\mathcal{E}}(10)
$$


The existence and uniqueness of $\boldsymbol{\mu}_{1}$ follows immediately from (55) which establishes the existence of $\left(1-C_{\mathcal{E}}\right)^{-1}$, and allows one to construct $\boldsymbol{\mu}_{1}$ by Neumann series, moreover, we have

$$
\left\|\boldsymbol{\mu}_{1}\right\|_{L^{2}\left(\Sigma^{(\mathcal{E})}\right)} \lesssim \frac{\left\|C_{\mathcal{E}}\right\|_{L^{2}\left(\Sigma^{(\mathcal{E})}\right) \rightarrow L^{2}\left(\Sigma^{(\mathcal{E})}\right)}}{1-\left\|C_{\mathcal{E}}\right\|_{L^{2}\left(\Sigma^{(\mathcal{E})}\right) \rightarrow L^{2}\left(\Sigma^{(\mathcal{E})}\right)}} \lesssim|t|^{-1 / 2} .
$$

Fix a small constant $d$ and suppose that $\inf _{z \in \Sigma^{(\mathcal{E})}}|\lambda-z|>d$, then

$$
\left|\mathbf{e}_{1}-\left(\begin{array}{ll}
1 & 0
\end{array}\right)\right| \leqslant \frac{d^{-1}}{2 \pi}\left(\left\|v^{(\mathcal{E})}-I\right\|_{L^{1}}+\left\|\boldsymbol{\mu}_{1}\right\|_{L^{2}}\left\|v^{(\mathcal{E})}-I\right\|_{L^{2}}\right) \lesssim|t|^{-1 / 2} .
$$

To get $L^{\infty}$ control for $\lambda$ approaching $\Sigma^{(\mathcal{E})}$ we observe that the jumps on the contours $\Sigma^{(\mathcal{E})}$ are locally analytic, and so the contour $\Sigma^{(\mathcal{E})}$ can be freely deformed to a new contour $\widetilde{\Sigma}^{(\mathcal{E})}$, with different points of self-intersection, by a bounded invertible transformation $\mathbf{e}_{1} \mapsto \widetilde{\mathbf{e}}_{1}$. The previous argument then goes through to show that $\left|\widetilde{\mathbf{e}}_{1}-\left(\begin{array}{ll}1 & 0\end{array}\right)\right|$ is bounded on $\Sigma^{(\mathcal{E})}$ which then gives a similar bound on $\mathbf{e}_{1}$ as the transformation itself is bounded.

To build the second row $\mathbf{e}_{2}=\left(\begin{array}{ll}\mathcal{E}_{21} & \mathcal{E}_{22}\end{array}\right)$, we begin by using the symmetry condition to compute $\bar{q}_{\mathcal{E}}$. Since $\mathcal{E}_{21}(\lambda)=\varepsilon \lambda \overline{\mathcal{E}_{12}(\bar{\lambda})}$ for all large $\lambda$, we use (57) and take the limit as $\lambda \rightarrow \infty$ to find

$$
q_{\mathcal{E}}=\frac{\varepsilon}{2 \pi i}\left(\int_{\Sigma^{(\mathcal{E})}}\left[(10)+\mathbf{e}_{1}(z)\right]\left[v^{(\mathcal{E})} z-I\right]_{2} d z\right)
$$

where the subscript 2 on the second factor of the integrand denotes the second column of the matrix. Finally, using (54) and (58) we have the bound

$$
\left|\bar{q}_{\mathcal{E}}\right| \lesssim|t|^{-1 / 2} \text {. }
$$

Now that $\bar{q}_{\mathcal{E}}$ is well defined, we construct the second row as

$$
\mathbf{e}_{2}(\lambda)=\left(\bar{q}_{\mathcal{E}} 1\right)+\frac{1}{2 \pi i} \int_{\Sigma^{(\mathcal{E})}} \frac{\left(\left(\bar{q}_{\mathcal{E}} 1\right)+\boldsymbol{\mu}_{2}(z)\right)\left(v^{(\mathcal{E})}(z)-I\right)}{z-\lambda} d z
$$

where

$$
\left(1-C_{\mathcal{E}}\right) \boldsymbol{\mu}_{2}=C_{\mathcal{E}}\left(\bar{q}_{\mathcal{E}} 1\right)
$$

Then repeating the arguments above we have that $\left\|\boldsymbol{\mu}_{2}\right\|_{L^{2} \Sigma^{(\mathcal{E})}} \lesssim|t|^{-1 / 2}$ and also that $\left\|\mathbf{e}_{2}-\left(\bar{q}_{\mathcal{E}} 1\right)\right\|_{L^{\infty}(\mathbb{C})} \lesssim|t|^{-1 / 2}$.

Define the matrices $\mathcal{E}=\left(\begin{array}{l}\mathbf{e}_{1} \\ \mathbf{e}_{2}\end{array}\right)$ and $\boldsymbol{\mu}=\left(\begin{array}{c}\boldsymbol{\mu}_{1} \\ \boldsymbol{\mu}_{2}\end{array}\right)$. Then, for large $\lambda$ write $\mathcal{E}_{0}=\left(\begin{array}{cc}1 & 0 \\ \bar{q}_{\mathcal{E}} & 1\end{array}\right)$ and

$$
\begin{gathered}
\mathcal{E}(\lambda)=\mathcal{E}_{0}+\lambda^{-1} \mathcal{E}_{1}+\lambda^{-2} \mathcal{S}(\lambda) \\
\mathcal{E}_{1}=\frac{-1}{2 \pi i} \int_{\Sigma^{(\mathcal{E})}}\left(\mathcal{E}_{0}+\boldsymbol{\mu}(z)\right)\left(v^{(\mathcal{E})}(z)-I\right) d z, \\
\mathcal{S}(\lambda)=\frac{\lambda}{2 \pi i} \int_{\Sigma^{(\mathcal{E})}} \frac{\left(\mathcal{E}_{0}+\boldsymbol{\mu}(z)\right) z\left(v^{(\mathcal{E})}(z)-I\right)}{(z-\lambda)} d z .
\end{gathered}
$$


Using (54) and (58), as $\lambda \rightarrow \infty$ the residual $\mathcal{S}$ satisfies

$$
|\mathcal{S}| \lesssim\left\|V_{E}-I\right\|_{L^{2,2}(\mathbb{R})} \lesssim|t|^{-1 / 2},
$$

while using (59) we have

$$
\begin{aligned}
\mathcal{E}_{1} & =-\frac{1}{2 \pi i} \oint_{\partial \mathcal{U}_{\xi}}\left(v^{(\mathcal{E})}(z)-I\right) d z+\mathcal{O}\left(|t|^{-1}\right) \\
& =|8 t|^{-1 / 2} \mathcal{N}^{\text {out }}(\xi) A(\xi, \eta) \mathcal{N}^{\text {out }}(\xi)^{-1}+\mathcal{O}\left(|t|^{-1}\right) .
\end{aligned}
$$

The last equality in (60) follows from a residue calculation using (46). A direct calculation using the fact that $\operatorname{det} \mathcal{N}^{\text {out }}=1$ then gives (56).

Combining Lemmas 3.7, 3.13, and 3.15, it follows from (51) that

Proposition 3.16. Let $c_{1}$ and $c_{2}$ be strictly positive constants, and suppose that $\rho \in H^{2,2}(\mathbb{R})$ with $\|\rho\|_{H^{2,2}(\mathbb{R})} \leqslant c_{1}$ and $\inf _{\lambda \in \mathbb{R}}\left(1-\varepsilon \lambda|\rho(\lambda)|^{2}\right) \geqslant c_{2}$. Then,

$$
\left\|\mathcal{N}^{\mathrm{RHP}}\right\|_{\infty} \lesssim 1 \quad\left\|\left(\mathcal{N}^{\mathrm{RHP}}\right)^{-1}\right\|_{\infty} \lesssim 1
$$

where the implied constants are uniform in $\xi$ and $|t|>1$ and depend only on $c_{1}$ and $c_{2}$.

When estimating the gauge factor for the solution $u$ of the DNLS equation (Section 4.2), we need the following result that provides the large-time behavior of the error term $\mathcal{E}$ at $z=0$ :

Proposition 3.17. Suppose that $\rho \in H^{2,2}(\mathbb{R})$ and $c:=\inf _{\lambda \in \mathbb{R}}\left(1-\varepsilon \lambda|\rho(\lambda)|^{2}\right)>0$ strictly. Then, as $|t| \rightarrow \infty$ the unique solution of RHP 3.14 described by Lemma 3.15 satisfies

$$
\mathcal{E}_{11}(0)=1-\frac{i \varepsilon}{|2 t|^{1 / 2}} \operatorname{Re}\left[A_{12} \mathcal{N}_{11}^{\text {out }}(\xi) \overline{\mathcal{N}_{12}^{\text {out }}(\xi)}\right]+\mathcal{O}\left(|t|^{-1}\right)
$$

whenever $0 \neq \mathcal{U}_{\xi} ;$ when $0 \in \mathcal{U}_{\xi}$ it satisfies

$$
\begin{aligned}
\mathcal{E}_{11}(0)= & 1-\frac{i \varepsilon}{|8 t|^{1 / 2}}\left[2 \operatorname{Re}\left(A_{12} \mathcal{N}_{11}^{\text {out }}(\xi) \overline{\mathcal{N}_{12}^{\text {out }}(\xi)}\right)-\overline{A_{12}} \mathcal{N}_{12}^{\text {out }}(0) \overline{\mathcal{N}_{11}^{\text {out }}(0)}\right] \\
& +\mathcal{O}\left(|t|^{-1}\right) \\
\mathcal{E}_{12}(0)= & \mathcal{O}\left(|t|^{-1 / 2}\right)
\end{aligned}
$$

where $A_{12}=A_{12}(\xi, \eta)$.

Proof. Write $\mathbf{e}_{1}=\left(\mathcal{E}_{11} \mathcal{E}_{12}\right)$ for the first row of $\mathcal{E}$. Then starting from (57) we use (52) and (46) together with the bounds (53), (54) and (58) to write

$$
\mathbf{e}_{1}(0)=\left(\begin{array}{ll}
1 & 0
\end{array}\right)-\frac{\left(\begin{array}{ll}
1 & 0
\end{array}\right)}{|8 t|^{1 / 2} 2 \pi i} \int_{\partial \mathcal{U}_{\xi}} \frac{\mathcal{N}^{\text {out }}(z) A(\xi, \eta) \mathcal{N}^{\text {out }}(z)^{-1}}{z(z-\xi)} d z+\mathcal{O}\left(|t|^{-1}\right) .
$$

A residue calculation, using the symmetry condition of RHP 3.5 and (48) to simplify the result, completes the proof. 
3.4. The remaining $\bar{\partial}$ problem. We are ready to consider the pure $\bar{\partial}$-Problem 3.2 for $n^{(3)}$. The next proposition describes its large-time asymptotics.

Proposition 3.18. Suppose that $\rho \in H^{2,2}(\mathbb{R})$ with $c:=\inf _{\lambda \in \mathbb{R}}\left(1-\lambda|\rho(\lambda)|^{2}\right)>0$ strictly. Then, for sufficiently large time $|t|>0$, there exists a unique solution $n^{(3)}(\lambda ; x, t)$ for $\bar{\partial}$-Problem 3.2 with the property that

$$
n^{(3)}(\lambda ; x, t)=I+\frac{1}{\lambda} n_{1}^{(3)}(x, t)+o_{\xi, t}\left(\frac{1}{\lambda}\right)
$$

for $\lambda=i y$ with $y \rightarrow+\infty$ where

$$
\left|n_{1}^{(3)}(x, t)\right| \lesssim|t|^{-3 / 4}
$$

where the implied constant in (65) is independent of $\xi$ and $t$ and uniform for $\rho$ in a bounded subset of $H^{2,2}(\mathbb{R})$ with $\inf _{\lambda \in \mathbb{R}}\left(1-\lambda|\rho(\lambda)|^{2}\right) \geqslant c>0$ for a fixed $c>0$.

Proposition 3.19. Given the same assumptions as Proposition 3.18 and for suffciently large times $|t|>0$, the unique solution $n^{(3)}(\lambda ; x, t)$ of $\bar{\partial}$-Problem 3.2 satisfies

$$
n_{11}^{(3)}(0 ; x, t)=1+\mathcal{O}\left(|t|^{-3 / 4}\right)
$$

where the implied constant is independent of $\xi$ and $t$ and is uniform for $\rho$ in a bounded subset of $H^{2,2}(\mathbb{R})$ with $\inf _{\lambda \in \mathbb{R}}\left(1-\lambda|\rho(\lambda)|^{2}\right) \geqslant c>0$ for a fixed $c>0$.

Remark 3.20. The remainder estimate in (64) need not be (and is not) uniform in $\xi$ and $t$; what matters for the proof of Theorem 1.5 is that the implied constant in the estimate (65) for $n_{1}^{(3)}(x, t)$ is independent of $\xi$ and $t$.

To prove Proposition 3.18 we recast $\bar{\partial}$-Problem 3.2 as a Fredholm-type integral equation using the solid Cauchy transform

$$
(P f)(\lambda)=\frac{1}{\pi} \int_{\mathbb{C}} \frac{1}{\lambda-z} f(z) d m(z)
$$

where $d m$ denotes Lebesgue measure on $\mathbb{C}$. The following lemma is standard.

Lemma 3.21. A continuous, bounded row vector-valued function $n^{(3)}(\lambda ; x, t)$ solves $\bar{\partial}$ Problem 3.2 if and only if

$$
n^{(3)}(\lambda ; x, t)=(1,0)+\frac{1}{\pi} \int_{\mathbb{C}} \frac{1}{\lambda-z} n^{(3)}(z ; x, t) W^{(3)}(z ; x, t) d m(z) .
$$

Proof of Proposition 3.18, given Lemmas 3.22-3.24. As in [1] and [9], we first show that, for large times, the integral operator $K_{W}$ defined by

$$
\left(K_{W} f\right)(\lambda)=\frac{1}{\pi} \int_{\mathbb{C}} \frac{1}{\lambda-z} f(z) W^{(3)}(z) d m(z)
$$

(suppressing the parameters $x$ and $t$ ) obeys the estimate

$$
\left\|K_{W}\right\|_{L^{\infty} \rightarrow L^{\infty}} \lesssim|t|^{-1 / 4}
$$

where the implied constants depend only on $\|\rho\|_{H^{2,2}}$ and

$$
c:=\inf _{\lambda \in \mathbb{R}}\left(1-\varepsilon \lambda|\rho(\lambda)|^{2}\right)
$$


and, in particular, are independent of $\xi$ and $t$. This is the object of Lemma 3.22. In particular, this shows that the solution formula

$$
n^{(3)}=\left(I-K_{W}\right)^{-1}(1,0)
$$

makes sense and defines an $L^{\infty}$ solution of (66) bounded uniformly in $\xi \in \mathbb{R}$ and $\rho$ in a bounded subset of $H^{2,2}(\mathbb{R})$ with $c>0$.

We then prove that the solution $n^{(3)}(\lambda ; x, t)$ has a large- $\lambda$ asymptotic expansion of the form (64) where $\lambda \rightarrow \infty$ along the positive imaginary axis (Lemma 3.23). Note that, for such $\lambda$, we can bound $|\lambda-z|$ below by a constant times $|\lambda|+|z|$. The remainder need not be bounded uniformly in $\xi$. Finally, we prove estimate (65).

Proof of Proposition 3.19, given Lemmas 3.22-3.24. From (66) we have

$$
n_{11}^{(3)}(0)=1-\frac{1}{\pi} \int_{\mathbb{C}} \frac{n_{11}^{(3)}(z) W_{11}^{(3)}(z)+n_{12}^{(3)}(z) W_{21}^{(3)}(z)}{z} d m(z) .
$$

Computing $W_{11}^{(3)}$ and $W_{21}^{(3)}$ using (38) and the symmetry in RHP 3.5, recalling that $\bar{\partial} \mathcal{R}^{(2)}$ has zeros on its diagonal, gives

$$
\begin{aligned}
& \frac{W_{11}^{(3)}(z)}{z}=\mathcal{N}_{12}^{\text {out }}(z) \overline{\mathcal{N}_{11}^{\text {out }}(\bar{z})} \frac{\bar{\partial} \mathcal{R}_{21}^{(2)}(z)}{z}+\varepsilon \mathcal{N}_{11}^{\text {out }}(z) \overline{\mathcal{N}_{12}^{\text {out }}(\bar{z})} \bar{\partial} \mathcal{R}_{12}^{(2)}(z) . \\
& \frac{W_{21}^{(3)}(z)}{z}={\overline{\mathcal{N}_{11}^{\text {out }}(\bar{z})}}^{2} \frac{\bar{\partial} \mathcal{R}_{21}^{(2)}(z)}{z}-z{\overline{\mathcal{N}_{12}^{\text {out }}(\bar{z})}}^{2} \bar{\partial} \mathcal{R}_{12}^{(2)}(z)
\end{aligned}
$$

Equations (67) and (68) imply that $\left|n^{(3)}(z)\right| \lesssim 1$. Using Lemma 3.7 then gives

$$
\left|n^{(3)}(0)-1\right| \lesssim \int_{\mathbb{C}}\left|\frac{\bar{\partial} \mathcal{R}_{21}^{(2)}(z)}{z}\right|+\left|\bar{\partial} \mathcal{R}_{12}^{(2)}(z)\right| d m(z)=\mathcal{O}\left(|t|^{-3 / 4}\right) .
$$

Where the last equality uses Corollary 2.5 to control the size of each term in the integrand, allowing identical estimates as those used to bound $\int\left|W^{(3)}(z)\right| d m(z)$ in Proposition 3.18 to establish the result.

Estimates (64), (65), and (67) rest on the bounds stated in three lemmas.

Lemma 3.22. Suppose that $\rho \in H^{2,2}(\mathbb{R})$ and

$$
c:=\inf _{\lambda \in \mathbb{R}}\left(1-\varepsilon \lambda|\rho(\lambda)|^{2}\right)>0
$$

strictly. Then, the estimate (67) holds, where the implied constants depend on $\|\rho\|_{H^{2,2}}$ and $c$.

Proof. To prove (67), first note that

$$
\left\|K_{W} f\right\|_{\infty} \leqslant\|f\|_{\infty} \int_{\mathbb{C}} \frac{1}{|\lambda-z|}\left|W^{(3)}(z)\right| d m(z)
$$

where, using Proposition 3.16,

$$
\left|W^{(3)}(z)\right| \leqslant\left\|\mathcal{N}^{\mathrm{RHP}}\right\|_{\infty}\left\|\left(\mathcal{N}^{\mathrm{RHP}}\right)^{-1}\right\|_{\infty}\left|\bar{\partial} \mathcal{R}^{(2)}\right| \lesssim\left|\bar{\partial} \mathcal{R}^{(2)}\right| .
$$

We will prove the estimate for $\eta=\eta=+1$ and $z \in \Omega_{1}$ since estimates for $\eta=-1$ and $\Omega_{3}, \Omega_{4}$, and $\Omega_{6}$ are similar. Setting $\lambda=\alpha+i \beta$ and $z-\xi=u+i v$, the region $\Omega_{1}$ corresponds to

$$
\Omega_{1}=\{(\xi+u, v): v \geqslant 0, v \leqslant u<\infty\} .
$$


We then have from Corollary 2.5 that

$$
\int_{\Omega_{1}} \frac{1}{|\lambda-z|}\left|W^{(3)}(z)\right| d m(z) \lesssim I_{1}+I_{2}+I_{3}+I_{4}
$$

where

$$
\begin{aligned}
& I_{1}=\int_{0}^{\infty} \int_{v}^{\infty} \frac{1}{|\lambda-z|}\left|p_{1}^{\prime}(u)\right| e^{-8 t u v} d u d v \\
& I_{2}=\int_{0}^{1} \int_{v}^{1} \frac{1}{|\lambda-z|}\left|\log \left(u^{2}+v^{2}\right)\right| e^{-8 t u v} d u d v \\
& I_{3}=\int_{0}^{\infty} \int_{v}^{\infty} \frac{1}{|\lambda-z|} \frac{1}{1+|z-\xi|} e^{-8 t u v} d u d v \\
& I_{4}=\int_{0}^{\infty} \int_{v}^{\infty} \frac{1}{|\lambda-z|}\left|\chi_{\Lambda}(z)\right| e^{-8 t u v} d u d v .
\end{aligned}
$$

We recall from [1, proof of Proposition C.1] the bound

$$
\left\|\frac{1}{\lambda-z}\right\|_{L^{2}(v, \infty)}=\left(\int_{v+\xi}^{\infty} \frac{d u}{(u-\alpha)^{2}+(v-\beta)^{2}}\right)^{1 / 2} \leqslant \frac{\pi^{1 / 2}}{|v-\beta|^{1 / 2}}
$$

where $z=\xi+u+i v$ and $\lambda=\alpha+i \beta$. Using this bound and Schwarz's inequality on the $u$-integration we may bound $I_{1}$ by constants times

$$
\left(1+\left\|p_{1}^{\prime}\right\|_{2}\right) \int_{0}^{\infty} \frac{1}{|v-\beta|^{1 / 2}} e^{-t v^{2}} d v \lesssim t^{-1 / 4}
$$

(see for example [1, proof of Proposition C.1] for the estimate) For $I_{2}$, we remark that $\left|\log \left(u^{2}+v^{2}\right)\right| \lesssim 1+\left|\log \left(u^{2}\right)\right|$ and that $1+\left|\log \left(u^{2}\right)\right|$ is square-integrable on $[0,1]$. We can then argue as before to conclude that $I_{2} \lesssim t^{-1 / 4}$. Similarly, the inequality

$$
\frac{1}{1+|z-\xi|} \leqslant \frac{1}{1+u}
$$

and the finite support of $\chi_{\Lambda}$ shows that we can bound $I_{3}$ and $I_{4}$ in a similar way.

It now follows that

$$
\int_{\Omega_{1}} \frac{1}{|\lambda-z|}\left|W^{(3)}(z)\right| d m(z) \lesssim t^{-1 / 4}
$$

which, together with similar estimates for the integrations over $\Omega_{3}, \Omega_{4}$, and $\Omega_{6}$, proves (67).

Lemma 3.23. For $\lambda=i y$, as $y \rightarrow+\infty$, the expansion (64) holds with

$$
n_{1}^{(3)}(x, t)=\frac{1}{\pi} \int_{\mathbb{C}} n^{(3)}(z ; x, t) W^{(3)}(z ; x, t) d m(z) .
$$

Proof. We write (66) as

$$
n^{(3)}(\lambda ; x, t)=(1,0)+\frac{1}{\lambda} n_{1}^{(3)}(x, t)+\frac{1}{\pi \lambda} \int_{\mathbb{C}} \frac{z}{\lambda-z} n^{(3)}(z ; x, t) W^{(3)}(z ; x, t) d m(z)
$$

where $n_{1}^{(3)}$ is given by (70). If $\lambda=i y$ and $z \in \Omega_{1} \cup \Omega_{3} \cup \Omega_{4} \cup \Omega_{6}$, it is easy to see that $|\lambda| /|\lambda-z|$ is bounded above by a fixed constant independent of $\lambda$, while 
$\left|n^{(3)}(z ; x, t)\right| \lesssim 1$ by the remarks following (68). If we can show that

$$
\int_{\mathbb{C}}\left|W^{(3)}(z ; x, t)\right| d m(z)<\infty
$$

it will follow from the Dominated Convergence Theorem that

$$
\lim _{y \rightarrow \infty} \int_{\mathbb{C}} \frac{z}{i y-z} n^{(3)}(z ; x, t) W^{(3)}(z ; x, t) d m(z)=0
$$

which implies the required asymptotic estimate. We will estimate the integral $\int_{\Omega_{1}}\left|W^{(3)}(z)\right| d m(z)$ since the other estimates are similar. Using Corollary 2.5 and (69), we may then estimate

$$
\int_{\Omega_{1}}\left|W^{(3)}(z ; x, t)\right| d m(z) \lesssim I_{1}+I_{2}+I_{3}+I_{4}
$$

where for $\eta=\operatorname{sgn} t=1$,

$$
\begin{array}{ll}
I_{1}=\int_{0}^{\infty} \int_{v}^{\infty}\left|p_{1}^{\prime}(\xi+u)\right| e^{-8 t u v} d u d v & I_{2}=\int_{0}^{1} \int_{v}^{1}\left|\log \left(u^{2}+v^{2}\right)\right| e^{-8 t u v} d u d v \\
I_{3}=\int_{0}^{\infty} \int_{v}^{\infty} \frac{1}{\sqrt{1+u^{2}+v^{2}}} e^{-8 t u v} d u d v & I_{4}=\int_{0}^{\infty} \int_{v}^{\infty}\left|\chi_{\Lambda}(z)\right| e^{-8 t u v} d u d v .
\end{array}
$$

For $\eta=-1$, the integrations domains are reflected in the $u$-variable, and the following estimates are altered in an obvious manner. To estimate $I_{1}$, we use the Schwarz inequality on the $u$-integration to obtain

$$
I_{1} \leqslant\left\|p_{1}^{\prime}\right\|_{2} \frac{1}{4 \sqrt{t}} \int_{0}^{\infty} \frac{1}{\sqrt{v}} e^{-8 t v^{2}} d v=\left\|p_{1}^{\prime}\right\|_{2} \frac{\Gamma(1 / 4)}{8^{5 / 4} t^{3 / 4}} .
$$

Since $\log \left(u^{2}+v^{2}\right) \leqslant \log \left(2 u^{2}\right)$ for $v \leqslant u \leqslant 1$, we may similarly bound

$$
I_{2} \leqslant\left\|\log \left(2 u^{2}\right)\right\|_{L^{2}(0,1)} \frac{\Gamma(1 / 4)}{8^{5 / 4} t^{3 / 4}} .
$$

To estimate $I_{3}$, we note that $1+u^{2}+v^{2} \geqslant 1+u^{2}$ and $\left(1+u^{2}\right)^{-1 / 2} \in L^{2}\left(\mathbb{R}^{+}\right)$, so we may conclude that

$$
I_{3} \leqslant\left\|\left(1+u^{2}\right)^{-1 / 2}\right\|_{2} \frac{\Gamma(1 / 4)}{8^{5 / 4} t^{3 / 4}} .
$$

Finally, as $\chi_{\Lambda}$ is finitely supported, by similar bounds

$$
I_{4} \leqslant C_{\Lambda} \frac{\Gamma(1 / 4)}{8^{5 / 4} t^{3 / 4}}
$$

where the constant $C_{\Lambda}$ depends only on the discrete spectrum $\Lambda$. These estimates together show that

$$
\int_{\Omega_{1}}\left|W^{(3)}(z ; x, t)\right| d m(z) \lesssim t^{-3 / 4}
$$

and that the implied constant depends only on $\|\rho\|_{H^{2,2}}$ and $\Lambda$. In particular, the integral (71) is bounded uniformly as $t \rightarrow \infty$.

The estimate (71) is also strong enough to prove (65):

Lemma 3.24. The estimate (65) holds with constants uniform in $\rho$ in a bounded subset of $H^{2,2}(\mathbb{R})$ and $\inf _{\lambda \in \mathbb{R}}\left(1-\varepsilon \lambda|\rho(\lambda)|^{2}\right)>0$ strictly. 
Proof. From the representation formula (70), Lemma 3.22, and the remarks following, we have

$$
\left|n_{1}^{(3)}(x, t)\right| \lesssim \int_{\mathbb{C}}\left|W^{(3)}(z ; x, t)\right| d m(z) .
$$

In the proof of Lemma 3.23 , we bounded this integral by $t^{-3 / 4}$ modulo constants with the required uniformities.

\section{LARGE-Time ASYMPtotics FOR SOLUtions of DNLS}

We now gather the estimates on the RHPs considered previously to prove Theorems 1.5 and 1.6 which provide precise asymptotic descriptions of the large-time behavior of the solutions $q(x, t)$ and $u(x, t)$ of (2a) and (1a) within any given spacetime cone $\mathcal{S}$. The leading-order soliton component of each expansion arises from our outer model $\mathcal{N}^{\text {out }}$. We begin with the following result, which describes the soliton components in $\mathcal{N}^{\text {out }}$ in terms of only those solitons whose speeds are 'visible' from $\mathcal{S}$.

Recall the notation (11), (15)-(18) used in Theorem 1.5-1.6 and for any real interval $I$ let

$$
\nu_{0}(I)=\nu_{0}=\min _{\lambda \in \Lambda \backslash \Lambda(I)} \operatorname{dist}(\operatorname{Re} \lambda, I) .
$$

Additionally, for any multi-soliton $q_{\mathrm{sol}}(x, t ; \mathcal{D})$, where $\mathcal{D}$ is the associated reflectionless scattering data, we use (78) below to define

$$
u_{\mathrm{sol}}(x, t ; \mathcal{D}):=q_{\mathrm{sol}}(x, t ; \mathcal{D}) \exp \left(-i \varepsilon \int_{x}^{\infty}\left|q_{\mathrm{sol}}(y, t ; \mathcal{D})\right|^{2} d y\right)
$$

Proposition 4.1. Fix $x_{1} \leqslant x_{2}$ and $v_{1} \leqslant v_{2}$. Take $\mathcal{S}$, I, and $\mathcal{D}_{I}$ as in Theorem 1.5; take $\mathcal{N}^{\text {out }}$ and $\mathcal{D}_{\xi}$ as in Proposition 3.6. Then as $|t| \rightarrow \infty$ with $(x, t) \in$ $\mathcal{S}\left(v_{1}, v_{2}, x_{1}, x_{2}\right)$ we have

$$
\mathcal{N}^{\text {out }}(\lambda)=\mathcal{N}^{\text {sol }}\left(\lambda \mid \mathcal{D}_{I}\right) \prod_{\operatorname{Re} \lambda_{k} \in I_{\xi, \eta}^{-} \backslash I}\left(\frac{\lambda-\bar{\lambda}_{k}}{\lambda-\lambda_{k}}\right)^{\sigma_{3}}+\mathcal{O}\left(e^{-4 \mathrm{~d}_{\Lambda} \nu_{0}|t|}\right)
$$

where $\mathcal{N}^{\text {sol }}\left(\lambda \mid \mathcal{D}_{I}\right)$ is the solution of RHP 1.2 corresponding to the reflectionless scattering data $\mathcal{D}_{I}$. In particular,

$$
\begin{aligned}
& 2 i\left(\mathcal{N}_{1}^{\text {out }}\right)_{12}:=\lim _{\lambda \rightarrow \infty} 2 i \lambda \mathcal{N}_{12}^{\text {out }}(\lambda ; x, t)=q_{\mathrm{sol}}\left(x, t ; \mathcal{D}_{I}\right)+\mathcal{O}\left(e^{-4 \mathrm{~d}_{\Lambda} \nu_{0}|t|}\right) \\
& \mathcal{N}^{\text {out }}(0 ; x, t)=\exp \left(\frac{i \varepsilon \sigma_{3}}{2} \int_{x}^{\infty}\left|q_{\mathrm{sol}}\left(y, t ; \mathcal{D}_{I}\right)\right|^{2} d y\right) \\
& \quad \times\left(\begin{array}{cc}
1 & -\int_{x}^{\infty} u_{\mathrm{sol}}\left(y, t ; \mathcal{D}_{I}\right) d y \\
0 & 1
\end{array}\right) \exp \left(\begin{array}{c}
-2 i \sigma_{3} \sum_{\operatorname{Re} \lambda_{k} \in I_{\xi, \eta}^{-} \backslash I} \arg \lambda_{k} \\
0
\end{array}\right)+\mathcal{O}\left(e^{-4 \mathrm{~d}_{\Lambda} \nu_{0}|t|}\right)
\end{aligned}
$$

Proof. Apply Lemma B.4 and Corollary B.5 of Appendix B to $\mathcal{N}^{\text {out }}$-as described by Proposition 3.6-and observe that $\mathcal{D}_{I}=\widehat{\mathcal{D}_{\xi}}$.

4.1. Large-time asymptotic for solution $q$ of (1.3). Inverting the sequence of transformations (29), (32), (36) we construct the solution $n$ as

$$
n(\lambda)=n^{(3)}(\lambda) \mathcal{N}^{\mathrm{RHP}}(\lambda) \mathcal{R}^{(2)}(\lambda)^{-1} \delta(\lambda)^{\sigma_{3}} .
$$


It follows from (28) and Corollary 2.5 that as $\lambda \rightarrow \infty$ non-tangentially to the real axis,

$$
\delta(\lambda)^{\sigma_{3}}=I+\left(\delta_{1} / \lambda\right) \sigma_{3}+\mathcal{O}\left(\lambda^{-2}\right), \quad \mathcal{R}^{(2)}=I+\mathcal{O}\left(e^{-c|t|}\right) .
$$

From (51), we have

$$
\mathcal{N}^{\mathrm{RHP}}(\lambda)=\mathcal{E}(\lambda) \mathcal{N}^{\text {out }}(\lambda)
$$

and the large- $\lambda$ behavior of $\mathcal{E}(\lambda)$ and $\mathcal{N}^{\text {out }}(\lambda)$ are given in Proposition 3.6 and Lemma 3.15 as

$$
\begin{array}{cl}
\mathcal{E}(\lambda)=\mathcal{E}_{0}+\lambda^{-1} \mathcal{E}_{1}+\mathcal{O}\left(\lambda^{-2}\right), & \mathcal{E}_{0}=\left(\begin{array}{cc}
1 & 0 \\
\bar{q}_{\mathcal{E}} & 1
\end{array}\right), \\
\mathcal{N}^{\text {out }}(\lambda)=\mathcal{N}_{0}^{\text {out }}+\lambda^{-1} \mathcal{N}_{1}^{\text {out }}+\mathcal{O}\left(\lambda^{-2}\right), & \mathcal{N}_{0}^{\text {out }}=\left(\begin{array}{cc}
\frac{1}{\varepsilon\left(\mathcal{N}_{1}^{\text {out }}\right)_{12}} & 1
\end{array}\right) .
\end{array}
$$

Proof of Theorem 1.5. Inserting the above expansions into (77) and using Proposition 3.18 the reconstruction formula (14) gives

$$
q(x, y)=2 i\left(\mathcal{N}_{1}^{\text {out }}\right)_{12}+2 i\left(\mathcal{E}_{1}\right)_{12}+\mathcal{O}\left(|t|^{-3 / 4}\right) .
$$

Using (75) for the first term on the right-hand-side and (56) to identify $2 i\left(\mathcal{E}_{1}\right)_{12}$ with the correction factor $f(x, t)$ in Theorem 1.5 gives (19).

4.2. Large-time asymptotic for solution $u$ of (1.1). To prove Theorem 1.6 we construct the solution $u(x, t)$ of the DNLS equation (1a) with initial data $u_{0}$ by means of the inverse gauge transformation

$$
u(x, t)=\mathcal{G}^{-1}(q)(x, t):=q(x, t) \exp \left(-i \varepsilon \int_{x}^{\infty}|q(y, t)|^{2} d y\right)
$$

As we have the large-time behavior of $q(x, t)$ in hand, to compute the large-time behavior of $u(x, t)$ it will suffice to evaluate the large-time asymptotics of the expression

$$
\exp \left(-i \varepsilon \int_{x}^{\infty}|q(y, t)|^{2} d y\right)
$$

Proposition 4.2. Suppose that $q_{0} \in H^{2,2}(\mathbb{R})$ and that $q(x, t)$ solves $(2 \mathrm{a})$ with initial data $q_{0}$. Let $\left\{\rho,\left\{\left(\lambda_{k}, c_{k}\right)\right\}_{k=1}^{N}\right\}$ be the scattering data associated to $q_{0}$. Fix $\xi=-x /(4 t)$ and $M>0$. Fix real constants $v_{1} \leqslant v_{2}$ and $x_{1} \leqslant x_{2}$. Define $\mathcal{S}, I$ and $\mathcal{D}_{I}$ as described in Theorem 1.5 and take $\alpha_{0}$ as in Theorem 1.6. Then as $|t| \rightarrow \infty$ with $(x, t) \in \mathcal{S}\left(v_{1}, v_{2}, x_{1}, x_{2}\right)$ :

For $\xi \geqslant M|t|^{1 / 8}$, we have

$$
\begin{aligned}
& \exp \left(-i \varepsilon \int_{x}^{\infty}|q(y, t)|^{2} d y\right)= \\
& \quad\left[1+\frac{2 i \varepsilon}{|2 t|^{1 / 2}} \operatorname{Re}\left[A_{12}(\xi, \eta) \mathcal{N}_{11}^{\text {sol }}\left(\xi \mid \mathcal{D}_{I}\right) \overline{\mathcal{N}_{12}^{\text {sol }}\left(\xi \mid \mathcal{D}_{I}\right)} e^{i \phi(\xi)}\right]+\mathcal{O}\left(|t|^{-3 / 4}\right)\right] \\
& \quad \times \exp \left(-i \varepsilon \int_{x}^{\infty}\left|q_{\mathrm{sol}}\left(y, t ; \mathcal{D}_{I}\right)\right|^{2} d y\right) e^{i \alpha_{0}(\xi, \eta)},
\end{aligned}
$$

while for $\xi \leqslant M|t|^{-1 / 8}$, 


$$
\begin{aligned}
& \exp \left(-i \varepsilon \int_{x}^{\infty}|q(y, t)|^{2} d y\right)= \\
& F(\xi, t, \eta)\left[1+\frac{i \varepsilon}{|2 t|^{1 / 2}}\left\{2 \operatorname{Re}\left[A_{12}(\xi, \eta) \mathcal{N}_{11}^{\text {sol }}\left(\xi ; x, t \mid \mathcal{D}_{I}\right) \overline{\mathcal{N}_{12}^{\text {sol }}\left(\xi ; x, t \mid \mathcal{D}_{I}\right)} e^{i \phi(\xi)}\right]\right.\right.
\end{aligned}
$$

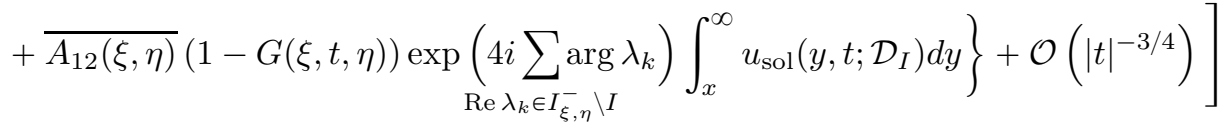

$$
\begin{aligned}
& \times \exp \left(-i \varepsilon \int_{x}^{\infty}\left|q_{\mathrm{sol}}\left(y, t ; \mathcal{D}_{I}\right)\right|^{2} d y\right) e^{i \alpha_{0}(\xi, \eta)}
\end{aligned}
$$

where, with $p:=e^{\frac{i \eta \pi}{4}}\left|8 t \xi^{2}\right|^{1 / 2}$

$$
F(\xi, t, \eta)=\left[e^{p^{2} / 4} p^{-i \eta \kappa(\xi)} D_{i \eta \kappa(\xi)}(p)\right]^{-2}, \quad G(\xi, t, \eta)=\frac{p D_{i \eta \kappa(\xi)-1}(p)}{D_{i \eta \kappa(\xi)}(p)},
$$

and $u_{\text {sol }}$ is defined by $(73)$.

Proof of Proposition 4.2. Using (93)-(94), the gauge factor (79) can be expressed in terms of spectral functions. Starting from $(77)$, observe that $\mathcal{R}^{(2)}(0)=\left(\begin{array}{ll}1 & * \\ 0 & 1\end{array}\right)$ is upper triangular, see Figure 3 and equation (33); similarly, the symmetry in condition 1 of RHP 3.1 guarantees that $\mathcal{N}_{21}^{\mathrm{RHP}}(0)=0$. So we have

$$
\begin{aligned}
& \exp \left(-i \varepsilon \int_{x}^{\infty}|q(y, t)|^{2} d y\right)=n_{11}(0 ; x, t)^{-2}=\left[n_{11}^{(3)}(0) \mathcal{N}_{11}^{\mathrm{RHP}}(0) \delta(0)\right]^{-2} \\
& \quad=\mathcal{N}_{11}^{\mathrm{RHP}}(0)^{-2} \exp \left(\frac{i}{\pi} \int_{I_{\xi, \eta}^{-}} \frac{\log \left(1-\varepsilon \lambda\left|\rho(\lambda)^{2}\right|\right)}{\lambda} d \lambda\right)+\mathcal{O}\left(|t|^{-3 / 4}\right)
\end{aligned}
$$

where we've used (26) and Proposition 3.19 in the last equality.

The value of $\mathcal{N}_{11}^{\mathrm{RHP}}(0)$ depends on the location of the point $\xi$ in the spectral plane.

If $|\xi|>\mathrm{d}_{\Lambda} / 3$ then $0 \notin \mathcal{U}_{\xi}$ (cf. (40)), so it follows from (51) and (76) that

$$
\begin{aligned}
\mathcal{N}_{11}^{\mathrm{RHP}}(0)^{-2}= & \mathcal{E}_{11}(0)^{-2} \exp \left(-i \varepsilon \int_{x}^{\infty}\left|q_{\mathrm{sol}}\left(y, t, \mathcal{D}_{I}\right)\right|^{2} d y+4 i \sum_{\operatorname{Re} \lambda_{k} \in I_{\xi, \eta}^{-} \backslash I} \arg \lambda_{k}\right) \\
& +\mathcal{O}\left(e^{-4 \mathrm{~d}_{\Lambda} \nu_{0}|t|}\right) .
\end{aligned}
$$

Plugging (84) into (83) and using (61) and (74) to evaluate $\mathcal{E}_{11}(0)$ gives (80).

If $|\xi|<\mathrm{d}_{\Lambda} / 3$ then $0 \in \mathcal{U}_{\xi}$. We expand (51), using Lemma 3.12 and (63) to drop terms of order $t^{-1}$ :

$$
\mathcal{N}_{11}^{\mathrm{RHP}}(0)=\mathcal{E}_{11}(0) \mathcal{N}_{11}^{\mathrm{PC}}(0) \mathcal{N}_{11}^{\text {out }}(0)\left(1+\frac{\mathcal{N}_{12}^{\text {out }}(0)}{\mathcal{N}_{11}^{\text {out }}(0)} \frac{\mathcal{N}_{21}^{\mathrm{PC}}(0)}{\mathcal{N}_{11}^{\mathrm{PC}}(0)}\right)+\mathcal{O}\left(|t|^{-1}\right)
$$

Using (76) this becomes

$$
\mathcal{N}_{11}^{\mathrm{RHP}}(0)^{-2}=\mathcal{E}_{11}(0)^{-2} \mathcal{N}_{11}^{\mathrm{PC}}(0)^{-2} \exp \left(-i \varepsilon \int_{x}^{\infty}\left|q_{\mathrm{sol}}\left(y, t, \mathcal{D}_{I}\right)\right|^{2} d y+\underset{\operatorname{Re} \lambda_{k} \in I_{\xi, \eta}^{-} \backslash I}{\arg } \lambda_{k}\right)
$$




$$
\times\left[1-\frac{\mathcal{N}_{21}^{\mathrm{PC}}(0)}{\mathcal{N}_{11}^{\mathrm{PC}}(0)} \exp \left(4 i \sum_{\operatorname{Re} \lambda_{k} \in I_{\xi, \eta}^{-} \backslash I} \arg \lambda_{k}\right) \int_{x}^{\infty} u_{\mathrm{sol}}\left(y, t ; D_{I}\right) d y+\mathcal{O}\left(|t|^{-1}\right)\right]^{-2} .
$$

Expanding $\mathcal{E}_{11}(0)^{-2}$ using (62) and Proposition 4.1

$$
\begin{aligned}
\mathcal{E}_{11}(0)^{-2}=1+ & \frac{i \varepsilon}{|2 t|^{1 / 2}}\left\{2 \operatorname{Re}\left[A_{12}(\xi, \eta) \mathcal{N}_{11}^{\mathrm{sol}, \varnothing}\left(\xi \mid \mathcal{D}_{I}\right) \overline{\mathcal{N}_{12}^{\mathrm{sol}, \varnothing}\left(\xi \mid \mathcal{D}_{I}\right)} e^{i \phi(\xi)}\right]\right. \\
& \left.+\overline{A_{12}(\xi, \eta)} \exp \left(4 i \sum_{\operatorname{Re} \lambda_{k} \in I_{\xi, \eta}^{-} \backslash I}^{\arg \lambda_{k}}\right) \int_{x}^{\infty} u_{\mathrm{sol}}\left(y, t ; \mathcal{D}_{I}\right) d y\right\}+\mathcal{O}\left(|t|^{-1}\right) .
\end{aligned}
$$

By introducing the notation $p:=e^{\frac{i \eta \pi}{4}}\left|8 t \xi^{2}\right|^{1 / 2}$, we use (50) and the symmetry $A_{21}=\varepsilon \xi \overline{A_{12}}$ (see (48)) to write

$$
\begin{gathered}
\mathcal{N}_{11}^{\mathrm{PC}}(0)^{-2}=\left[e^{p^{2} / 4} p^{-i \eta \kappa(\xi)} D_{i \eta \kappa(\xi)}(p)\right]^{-2}=F(\xi, t, \eta) \\
\frac{\mathcal{N}_{21}^{\mathrm{PC}}(0)}{\mathcal{N}_{11}^{\mathrm{PC}}(0)}=-i \varepsilon|\xi| e^{i \eta \pi / 4} \frac{D_{i \eta{ }_{12}(\xi)-1}(p)}{D_{i \eta \kappa(\xi)}(p)}=-\overline{A_{12}} i \varepsilon|8 t|^{-1 / 2} p \frac{D_{i \eta \kappa(\xi)-1}(p)}{D_{i \eta \kappa(\xi)}(p)} .
\end{gathered}
$$

Combining (85)-(86) with (83) gives (81).

Finally, we observe [10, Eq. 12.9.1] that inserting the expansion

$$
D_{\nu}(p)=e^{-p^{2} / 4} p^{\nu}\left[1+\mathcal{O}\left(p^{-2}\right)\right]
$$

into (82) gives

$$
F(p)=1+\mathcal{O}\left(|t|^{-1} \xi^{-2}\right) \quad G(p)=1+\mathcal{O}\left(|t|^{-1} \xi^{-2}\right)
$$

so that the inner expansion (81) for $|\xi| \leqslant \mathrm{d}_{\Lambda} / 3$ agrees with the outer expansion (80) for $|\xi|>M|t|^{-1 / 8}$.

\section{ACKNOWLEDGEMENTS}

The authors thank the referee for a very careful reading of the manuscript and a number of suggestions which helped us make the revised version more readable.

PAP and JL would like to thank the University of Toronto and the Fields Institute for hospitality during part of the time that this work was done. Conversely, RJ and CS would like to thank the department of Mathematics at the University of Kentucky for their hospitality during part of the time that this work was done.

PAP was supported in part by Simons Foundation Research and Travel Grant 359431, and CS was supported in part by Grant 46179-13 from the Natural Sciences and Engineering Research Council of Canada.

\section{Appendix A. The Weak Plancherel Formula}

We establish relations between the transmission coefficients $\breve{\alpha}$ and $\alpha$ and the scattering data $\left(\rho,\left\{\lambda_{k}\right\}_{k=1}^{N}\right)$. Recall that $\Lambda^{+}=\left\{\lambda_{k}\right\}_{k=1}^{N} \subset \mathbb{C}^{+}$.

Lemma A.1. The following relations

$$
\breve{\alpha}(\lambda)=\prod_{k=1}^{N} \frac{\lambda-\lambda_{k}}{\lambda-\bar{\lambda}_{k}} \exp \left(-\int_{-\infty}^{+\infty} \frac{\log \left(1-\varepsilon \xi|\rho(\xi)|^{2}\right)}{\xi-\lambda} \frac{d \xi}{2 \pi i}\right)
$$




$$
\alpha(\lambda)=\prod_{k=1}^{N} \frac{\lambda-\bar{\lambda}_{k}}{\lambda-\lambda_{k}} \exp \left(\int_{-\infty}^{+\infty} \frac{\log \left(1-\varepsilon \xi|\rho(\xi)|^{2}\right)}{\xi-\lambda} \frac{d \xi}{2 \pi i}\right)
$$

hold.

Proof. The functions $\breve{\alpha}(\lambda)$ and $\alpha(\lambda)$ have simple zeros in $\Lambda^{+}$and $\overline{\Lambda^{+}}$respectively. Defining

$$
\breve{\gamma}(\lambda)=\prod_{k=1}^{N} \frac{\lambda-\bar{\lambda}_{k}}{\lambda-\lambda_{k}} \breve{\alpha}(\lambda), \quad \gamma(\lambda)=\prod_{k=1}^{N} \frac{\lambda-\lambda_{k}}{\lambda-\bar{\lambda}_{k}} \alpha(\lambda),
$$

$\breve{\gamma}(\lambda)$ is analytic in the upper half plane where it has no zeros, while $\gamma$ is analytic in the lower half plane where it has no zeros. Also $\breve{\gamma}$ and $\gamma \rightarrow 1$ as $|\lambda| \rightarrow \infty$ in the respective half planes. Therefore,

$$
\begin{array}{rll}
\log \breve{\gamma}(\lambda)=\int_{-\infty}^{+\infty} \frac{\log \breve{\gamma}(\xi)}{\xi-\lambda} \frac{d \xi}{2 \pi i}, & \int_{-\infty}^{+\infty} \frac{\log \gamma(\xi)}{\xi-\lambda} \frac{d \xi}{2 \pi i}=0 & \operatorname{Im}(\lambda)>0 \\
\log \gamma(\lambda)=-\int_{-\infty}^{+\infty} \frac{\log \gamma(\xi)}{\xi-\lambda} \frac{d \xi}{2 \pi i}, & \int_{-\infty}^{+\infty} \frac{\log \breve{\gamma}(\xi)}{\xi-\lambda} \frac{d \xi}{2 \pi i}=0 & \operatorname{Im}(\lambda)<0 .
\end{array}
$$

Using (89), as well as the identity $\breve{\alpha}(\xi) \alpha(\xi)=\breve{\gamma}(\xi) \gamma(\xi)=\left(1-\xi|\rho(\xi)|^{2}\right)^{-1}$, we deduce

$$
\begin{array}{ll}
\log \breve{\alpha}(\lambda)=\sum_{k=1}^{N} \log \left(\frac{\lambda-\lambda_{k}}{\lambda-\bar{\lambda}_{k}}\right)-\int_{-\infty}^{+\infty} \frac{\log \left(1-\varepsilon \xi|\rho(\xi)|^{2}\right)}{\xi-\lambda} \frac{d \xi}{2 \pi i}, & \operatorname{Im}(\lambda)>0, \\
\log \alpha(\lambda)=\sum_{k=1}^{N} \log \left(\frac{\lambda-\bar{\lambda}_{k}}{\lambda-\lambda_{k}}\right)+\int_{-\infty}^{+\infty} \frac{\log \left(1-\varepsilon \xi|\rho(\xi)|^{2}\right)}{\xi-\lambda} \frac{d \xi}{2 \pi i}, & \operatorname{Im}(\lambda)>0 .
\end{array}
$$

from which the identities (87) and (88) are obtained.

The next lemma can be seen as a weak version of a Plancherel identity for the scattering transform. One should compare it to the following identity for the AKNS system associated the defocussing cubic NLS equation:

$$
-\int_{\mathbb{R}} \log \left(1-|r(k)|^{2}\right) d k=\pi\|q\|_{L^{2}}^{2}
$$

where $q$ is the potential and $r$ is its scattering transform (see, for example, the discussion in [25], Appendix A). For small data this reduces to the linear Plancherel formula. In our "weak Plancherel formula," we only obtain equality modulo $2 \pi$ because of the exponentials.

Lemma A.2. Suppose that $q(x, t)$ is the solution of (2a) for initial data $q_{0} \in$ $H^{2,2}(\mathbb{R})$ and let $\left\{\rho,\left\{\left(\lambda_{k}, c_{k}\right)\right\}_{k=1}^{N}\right\}$ be the scattering data associated to $q_{0}$. Then the identity

$$
\exp \left[i \varepsilon \int_{\mathbb{R}}|q(y, t)|^{2}\right]=\exp \left[-4 i\left(\sum_{k=1}^{N} \arg \lambda_{k}\right)-\frac{i}{\pi} \int_{\mathbb{R}} \frac{\log \left(1-\varepsilon \lambda|\rho(\lambda)|^{2}\right)}{\lambda} d \lambda\right]
$$

holds. 
Proof. We can express the transmission coefficient (88) in terms of the normalized Jost function matrices $N^{ \pm}(x, t ; \lambda)$ defined by

$$
\begin{aligned}
\frac{d N^{ \pm}}{d x} & =-i \lambda\left[\sigma_{3}, N^{ \pm}\right]+Q_{\lambda} N^{ \pm}-\frac{i}{2} \sigma_{3} Q^{2} N^{ \pm} \\
\lim _{x \rightarrow \pm \infty} N^{ \pm}(x, \lambda) & =I
\end{aligned}
$$

(see (4)) where $q(x)$ is replaced by $q(x, t)$ in the definitions of $Q$ and $Q_{\lambda}$. Recall that

$$
N^{+}(x, \lambda)=N^{-}(x, \lambda) e^{-i \lambda x \operatorname{ad}\left(\sigma_{3}\right)} T(\lambda) .
$$

where $T$ Using the definition (6) of $T$ and (91) and taking the limit as $x \rightarrow-\infty$ gives

$$
\alpha(\lambda)=\lim _{x \rightarrow-\infty} N_{11}^{+}(x, t ; \lambda) .
$$

Consider (90) for $\lambda \approx 0$

$$
\begin{aligned}
& \frac{\mathrm{d} N^{+}}{\mathrm{d} x}=\left(\begin{array}{cc}
-\frac{i \varepsilon}{2}|q(x, t)|^{2} & q(x, t) \\
0 & \frac{i \varepsilon}{2}|q(x, t)|^{2}
\end{array}\right) N^{+}+\lambda\left[\left(\begin{array}{cc}
\frac{-i}{\varepsilon q(x, t)} & i
\end{array}\right) N^{+}+N^{+}\left(\begin{array}{cc}
i & 0 \\
0 & -i
\end{array}\right)\right] . \\
& \lim _{x \rightarrow \infty} N^{+}(x, t ; \lambda)=I
\end{aligned}
$$

As $\lambda=0$ is a regular point of this system of equations, one can easily show that

$$
N^{+}(x, t ; \lambda)=e^{\frac{i \varepsilon \sigma_{3}}{2} \int_{x}^{\infty}|q(y, t)|^{2} d y}\left(\begin{array}{cc}
1 & -\int_{x}^{\infty} q(y, t) e^{-i \varepsilon \int_{y}^{\infty}|q(w, t)|^{2} d w} d y \\
0 & 1
\end{array}\right)+\mathcal{O}(\lambda) .
$$

Writing $N_{1}^{+}$for the first column of the Jost function we have

$$
N_{1}^{+}(x, t ; 0)=N_{1-}(0 ; x, t)=N_{1}(x, t, 0),
$$

where we have used the fact that the Jost function $N_{1}^{+}(x, t ; 0)$ gives the first column of the Beals-Coifman solution $N_{-}(0 ; x, t)$ of RHP 1.2 (see, for example, [21, Section 4.1] for discussion). We can drop the minus-boundary value because the jump relation in Problem 1.2(iii) gives $N_{11+}(0 ; x, t)=N_{11-}(0 ; x, t)$ so that $N_{11}(\lambda ; x, t)$ is continuous at the origin. Evaluating $\alpha(0)^{2}$ two ways: by combining (94) with (92); and evaluating (88) at $\lambda=0$, gives the result.

\section{Appendix B. Solutions of RHP 1.2 FOR REFleCtionless SCATTERING DATA}

The bright soliton solutions of (2a) can be characterized as the potentials $q(x, t)$ for which the associated scattering data are reflectionless: $\left(\rho \equiv 0,\left\{\left(\lambda_{k}, C_{k}\right)\right\}_{k=1}^{N}\right)$, and $\left(\lambda_{k}, C_{k}\right) \in \mathbb{C}^{+} \times \mathbb{C}^{\times}$for $k=1, \ldots, N$. If $N=1$, with scattering data $(\lambda=$ $u+i v, C)$, the single soliton solution of $(2 \mathrm{a})$ is

$$
\begin{aligned}
\mathcal{Q}_{\text {sol }}(x, t)= & \mathcal{Q}\left(x-x_{0}+4 u t, \lambda\right) \\
& \times \exp i\left\{4|\lambda|^{2} t-2 u(x+4 u t)-\frac{\varepsilon}{4} \int_{-\infty}^{x-x_{0}+4 u t} \mathcal{Q}(\eta, \lambda)^{2} d \eta-\varphi_{0}\right\}
\end{aligned}
$$

where

$$
\mathcal{Q}(y, \lambda)=\sqrt{\frac{8 v^{2}}{|\lambda| \cosh (4 v y)-\varepsilon u}},
$$




$$
x_{0}=\frac{1}{4 v} \log \frac{|\lambda \| C|^{2}}{4 v^{2}}, \quad \varphi_{0}=\arg (\lambda)+\arg (C)+\pi / 2,
$$

which describes a solitary wave with amplitude envelope $\mathcal{Q}$ traveling at speed $c=-4 \operatorname{Re} \lambda$. For $N>1$, the solution formulae become ungainly, but we expect, generically, that for $|t| \gg 1$, the solution will resemble $N$ independent 1-solitons each traveling at its unique speed $-4 \operatorname{Re} \lambda_{k}{ }^{2}$. For this reason, these solutions are called $N$-solitons of (2a).

Problem B.1. Given $(x, t) \in \mathbb{R}^{2}$ and data $\mathcal{D}=\left\{\left(\lambda_{k}, C_{k}\right)\right\}_{k=1}^{N} \subseteq \mathbb{C}^{+} \times \mathbb{C}^{\times}$fix $\Delta \subset\{1, \ldots, N\}$. Find an analytic function $\mathcal{N}^{\mathrm{sol}, \Delta}(\cdot ; x, t \mid \mathcal{D}):(\mathbb{C} \backslash \Lambda) \rightarrow S L_{2}(\mathbb{C})$ such that

(i) $\mathcal{N}^{\text {sol, } \Delta}$ satisfies the symmetry relation

$$
\mathcal{N}^{\mathrm{sol}, \Delta}(\lambda ; x, t \mid \mathcal{D})=\lambda^{-\sigma_{3} / 2} \sigma_{\varepsilon}^{-1} \overline{\mathcal{N}^{\mathrm{sol}, \Delta}(\bar{\lambda} ; x, t \mid \mathcal{D})} \sigma_{\varepsilon} \lambda^{\sigma_{3} / 2}
$$

(ii) $\mathcal{N}^{\text {sol }, \Delta}(\lambda ; x, t \mid \mathcal{D})=\left(\begin{array}{cc}1 & 0 \\ \alpha(x, t) & 1\end{array}\right)+\mathcal{O}\left(\lambda^{-1}\right)$ as $\lambda \rightarrow \infty$.

(iii) $\mathcal{N}^{\text {sol, }, \Delta}$ has simple poles at each point in $\Lambda$. For each $\lambda_{k} \in \Lambda_{+}$

$$
\begin{aligned}
& \operatorname{res}_{\lambda=\lambda_{k}} \mathcal{N}^{\mathrm{sol}, \Delta}(\lambda ; x, t \mid \mathcal{D})=\lim _{\lambda \rightarrow \lambda_{k}} \mathcal{N}^{\mathrm{sol}, \Delta}(\lambda ; x, t \mid \mathcal{D}) v^{\Delta}\left(\lambda_{k}\right) \\
& \operatorname{res}_{\lambda=\overline{\lambda_{k}}} \mathcal{N}^{\mathrm{sol}, \Delta}(\lambda ; x, t \mid \mathcal{D})=\lim _{\lambda \rightarrow \bar{\lambda}_{k}} \mathcal{N}^{\mathrm{sol}, \Delta}(\lambda ; x, t \mid \mathcal{D}) v^{\Delta}\left(\overline{\lambda_{k}}\right)
\end{aligned}
$$

where

$$
\begin{aligned}
& v^{\Delta}\left(\lambda_{k}\right)= \begin{cases}\left(\begin{array}{cc}
0 & 0 \\
\lambda_{k} \gamma_{k}^{\Delta}(x, t) & 0
\end{array}\right) & k \notin \Delta \\
\left(\begin{array}{cc}
0 & \lambda_{k}^{-1} \gamma_{k}^{\Delta}(x, t) \\
0 & 0
\end{array}\right) & k \in \Delta,\end{cases} \\
& v^{\Delta}\left(\overline{\lambda_{k}}\right)=\overline{\lambda^{-\sigma_{3} / 2} \sigma_{\varepsilon}^{-1} v^{\Delta}\left(\lambda_{k}\right) \sigma_{\varepsilon} \lambda^{\sigma_{3} / 2}},
\end{aligned}
$$

and

$$
\begin{gathered}
\gamma_{k}^{\Delta}(x, t)= \begin{cases}C_{k} \mathfrak{B}^{\Delta}\left(\lambda_{k}\right)^{-2} e^{-2 i t \theta\left(\lambda_{k}\right)} & k \notin \Delta \\
C_{k}^{-1}\left(1 / \mathfrak{B}^{\Delta}\right)^{\prime}\left(\lambda_{k}\right)^{-2} e^{2 i t \theta\left(\overline{\lambda_{k}}\right)} & k \in \Delta .\end{cases} \\
\mathfrak{B}^{\Delta}(\lambda)=\prod_{k \in \Delta}\left(\frac{\lambda-\bar{\lambda}_{k}}{\lambda-\lambda_{k}}\right)
\end{gathered}
$$

Remark B.2. When the context is clear we will omit the dependence of $\mathcal{N}^{\text {sol, } \Delta}$ on $x, t$, and/or $\mathcal{D}$ so that $\mathcal{N}^{\text {sol, }, \Delta}(\lambda)=\mathcal{N}^{\text {sol, }, \Delta}(\lambda, \mid \mathcal{D})=\mathcal{N}^{\text {sol, }, \Delta}(\lambda ; x, t \mid \mathcal{D})$ are all equivalent representations of the same function.

When $\Delta=\varnothing$ Problem B.1 is identical to RHP 1.2 with scattering data $\{\rho \equiv$ $\left.0, \mathcal{D}=\left\{\left(\lambda_{k}, C_{k}\right)\right\}_{k=1}^{N}\right\}$. For any other choice of $\Delta$ the relation between these problems is ${ }^{3}$

$$
\mathcal{N}^{\mathrm{sol}, \varnothing}(\lambda)=\mathcal{N}^{\mathrm{sol}, \Delta}(\lambda) \mathfrak{B}^{\Delta}(\lambda)^{\sigma_{3}}
$$

\footnotetext{
${ }^{2}$ The non-generic case occurs when $\operatorname{Re} \lambda_{j}=\operatorname{Re} \lambda_{k}$ for one or more pairs $j \neq k$. In this case the solution possesses localized, quasi-periodic traveling waves known as breather solitons.

${ }^{3}$ When $\Delta=\varnothing$ we set $\mathfrak{B} \varnothing(\lambda)=I$ for consistency.
} 
Each choice of $\Delta$ represents a different normalization of the RHP. The idea, which is common in the literature $[1,2,5]$, is to choose normalizations which prepare the problem for asymptotic analysis.

For convenience, order the spectrum (possibly non-uniquely) such that

$$
\operatorname{Re} \lambda_{1} \leqslant \operatorname{Re} \lambda_{2} \leqslant \cdots \leqslant \operatorname{Re} \lambda_{N},
$$

and let $\operatorname{Re} \lambda_{N+1}=-\operatorname{Re} \lambda_{0}=+\infty$. For $j=0,1, \ldots, N$, define the sets

$$
\begin{gathered}
S_{j}^{ \pm}=\left\{(x, t) \in \mathbb{R}^{2}: \xi \in\left[\operatorname{Re} z_{j}, \operatorname{Re} z_{j+1}\right), \pm t \geqslant 0\right\}, \\
\Delta_{j}^{+}=\{\ell \in \mathbb{N}: 1 \leqslant \ell \leqslant j\}, \quad \Delta_{j}^{-}=\{1, \ldots, N\} \backslash \Delta_{j}^{+}
\end{gathered}
$$

As $\eta t \rightarrow \infty$ with $(x, t) \in S_{j}^{\eta}$, the set of $\lambda_{k}$ which have exponentially growing residue coefficients in $\mathcal{N}^{\mathrm{sol}, \varnothing}$ are indexed by $\Delta_{j}^{\eta}$ - the remaining poles have bounded residues. The transformation (97) results in a new problem for $\mathcal{N}^{\text {sol, } \Delta_{j}^{\eta}}$ which has, in light of (13), and (96), residue coefficients $\gamma_{k}^{\Delta_{j}^{\eta}}(x, t)$ which are uniformly bounded as $(x, t)$ vary over $S_{j}^{\eta}$ :

$$
\begin{gathered}
\left|\gamma_{k}^{\Delta_{j}^{\eta}}(x, t)\right| \leqslant K_{\Lambda}\left\{\begin{array}{ll}
e^{-8|t| \operatorname{Im} \lambda_{k}\left|\operatorname{Re} \lambda_{k}-\xi\right|} & |\xi| \leqslant \Xi_{0} \\
e^{-2|x| \operatorname{Im} \lambda_{k}\left|1-\xi^{-1} \operatorname{Re} \lambda_{k}\right|} & |\xi| \geqslant \Xi_{0}
\end{array}\right\} \leqslant K_{\Lambda}, \\
\forall k,(x, t) \in S_{j}^{\eta}
\end{gathered}
$$

where $\Xi_{0}>0$ is any fixed constant and $K_{\Lambda}$ is a fixed constant depending only on the scattering data.

Lemma B.3. Given data $\mathcal{D}=\left\{\left(\lambda_{k}, C_{k}\right)\right\}_{k=1}^{N} \subset \mathbb{C}^{+} \times \mathbb{C}^{\times}$such that $\lambda_{j} \neq \lambda_{k}$ for $j \neq k$ there exists a unique solution of Problem B.1 for each $(x, t) \in \mathbb{R}^{2}$. Moreover, the solution satisfies

$$
\left\|\left(\mathcal{N}^{\mathrm{sol}, \Delta}\right)^{-1}\right\|_{L^{\infty}\left(\mathbb{C} \backslash \mathcal{B}_{\Lambda}\right)} \lesssim 1
$$

where $\mathcal{B}_{\Lambda}$ is any open neighborhood of the poles $\Lambda=\left\{\lambda_{k}, \overline{\lambda_{k}}\right\}_{k=1}^{N}$, and the implied constant depends only on $\mathcal{B}_{\Lambda}$ and the scattering data; it is independent of $x, t$ and $\Delta$.

Proof. Since $\operatorname{det} \mathcal{N}^{\text {sol, }, \Delta}(\lambda)=1$ we only need to consider $\left\|\mathcal{N}^{\text {sol, }, \Delta}\right\|$. The relation (97) is bounded and invertible away from $\Lambda$, so it is sufficient to work with whichever choice of $\Delta$ is convenient for any given $(x, t)$. As noted previously, taking $\Delta=\varnothing$, Problem B.1 is exactly RHP 1.2 for scattering data $\{\rho \equiv 0, \mathcal{D}\}$. Existence and uniqueness in the case $\varepsilon=-1$ follow from Theorem 4.3 of [17] and the equivalence of Problems 1.2 and 1.4. To prove boundedness, observe that the solution $\mathcal{N}^{\text {sol, } \Delta}$ of Problem B.1 is a continuous function of the parameters $\gamma_{k}^{\Delta}$. For $(x, t) \in S_{j}^{\eta}$, fix $\Delta=\Delta_{j}^{\eta}$, then (98) shows that the parameters $\gamma_{k}^{\Delta_{j}^{\eta}}$ vary over compact sets. This establishes boundedness on each $S_{j}^{\eta}$, and since $\bigcup_{j=0}^{N}\left(S_{j}^{+} \cup S_{j}^{-}\right)=\mathbb{R}^{2}$, this completes the proof.

Let us now consider the asymptotic behavior of soliton solutions which is needed for Theorems 1.5-1.6. Recall the notation established by (11), (15)-(18), and (72).

Lemma B.4. Fix reflectionless data $\mathcal{D}=\left\{\left(\lambda_{k}, C_{k}\right)\right\}_{k=1}^{N}$; parameters $v_{1} \leqslant v_{2}$, $x_{1} \leqslant x_{2}$, and a cone $\mathcal{S}\left(v_{1}, v_{2}, x_{1}, x_{2}\right)$ as in Theorem 1.5. Let $I=\left[-v_{2} / 4,-v_{1} / 4\right]$, 
$\eta=\operatorname{sgn} t$, and take $\Lambda^{+}(I)$ and $N(I) \leqslant N$ as in (16)-(17). Then, as $|t| \rightarrow \infty$ with $(x, t) \in \mathcal{S}\left(v_{1}, v_{2}, x_{1}, x_{2}\right)$,

$$
\mathcal{N}^{\mathrm{sol}, \varnothing}(\lambda \mid \mathcal{D})=\left[I+\mathcal{O}\left(e^{-4 \mathrm{~d}_{\Lambda} \nu_{0}|t|}\right)\right] \mathcal{N}^{\mathrm{sol}, \varnothing}(\lambda \mid \widehat{\mathcal{D}}) \prod_{\operatorname{Re} \lambda_{k} \in I_{\xi, \eta}^{-} \backslash I}\left(\frac{\lambda-\bar{\lambda}_{k}}{\lambda-\lambda_{k}}\right)^{\sigma_{3}}
$$

where

$$
\widehat{\mathcal{D}}=\left\{\left(\lambda_{k}, \widehat{C}_{k}\right): \lambda_{k} \in \Lambda(I), \quad \widehat{C}_{k}=\underset{\operatorname{Re} \lambda_{j} \in I_{\xi, \eta}^{-} \backslash I}{C_{k}}\left(\frac{\lambda_{k}-\lambda_{j}}{\lambda_{k}-\bar{\lambda}_{j}}\right)^{2}\right\} .
$$

Using (14) and (93)-(94) applied to $\mathcal{N}^{\text {sol, } \varnothing}(\lambda \mid \mathcal{D})$ we have the immediate corollary

Corollary B.5. Under the assumption of Lemma B.4 it follows that as $|t| \rightarrow \infty$ with $(x, t) \in \mathcal{S}\left(v_{1}, v_{2}, x_{1}, x_{2}\right)$ we have:

$$
\begin{aligned}
\lim _{\lambda \rightarrow \infty} 2 i \lambda \mathcal{N}_{12}^{\mathrm{sol}, \varnothing}(\lambda ; x, t \mid \mathcal{D}) & =q_{\mathrm{sol}}(x, t ; \mathcal{D})=q_{\mathrm{sol}}(x, t ; \widehat{\mathcal{D}})\left(1+e^{-4 \mathrm{~d}_{\Lambda} \nu_{0}|t|}\right) \\
\mathcal{N}_{11}^{\mathrm{sol}, \varnothing}(0 ; x, t \mid \mathcal{D})^{-2}= & \exp \left(-i \varepsilon \int_{x}^{\infty}\left|q_{\mathrm{sol}}(y, t ; \mathcal{D})\right|^{2} d y\right) \\
= & \exp \left(-i \varepsilon \int_{x}^{\infty}\left|q_{\mathrm{sol}}(y, t ; \widehat{\mathcal{D}})\right|^{2} d y+4 i \sum_{\operatorname{Re} \lambda_{k} \in I_{\xi, \eta}^{-} \backslash I} \arg \lambda_{k}\right) \\
& \times\left(1+e^{-4 \mathrm{~d}_{\Lambda} \nu_{0}|t|}\right) .
\end{aligned}
$$

Proof of Lemma B.4. Let $\Delta_{I}^{+}=\left\{k: \operatorname{Re} \lambda_{k}<-v_{2} / 4\right\}$ and $\Delta_{I}^{-}=\left\{k: \operatorname{Re} \lambda_{k}>\right.$ $\left.-v_{1} / 4\right\}$. Consider Problem B.1 with $\Delta=\Delta_{I}^{\eta}$. For $\lambda_{j} \in \Lambda \backslash \Lambda(I)$ and $(x, t) \in \mathcal{S}$ the residue coefficients (96) satisfy

$$
\left\|v^{\Delta_{I}^{ \pm}}\left(\lambda_{j}\right)\right\| \sim e^{-8 t \operatorname{Im} \lambda_{j} \operatorname{Re}\left(\lambda_{j}-\xi\right)}=\mathcal{O}\left(e^{-4 \mathrm{~d}_{\Lambda} \nu_{0}|t|}\right), \quad \pm t \rightarrow+\infty .
$$

Introduce small disks $D_{k}$ around each $\lambda_{k} \in \Lambda^{+} \backslash \Lambda(I)$ whose radii are chosen sufficiently small that they are non-overlapping and do not intersect the real axis. Orient their boundaries, $\partial D_{k}$, positively. We trade these residues for near-identity jumps by the change of variables

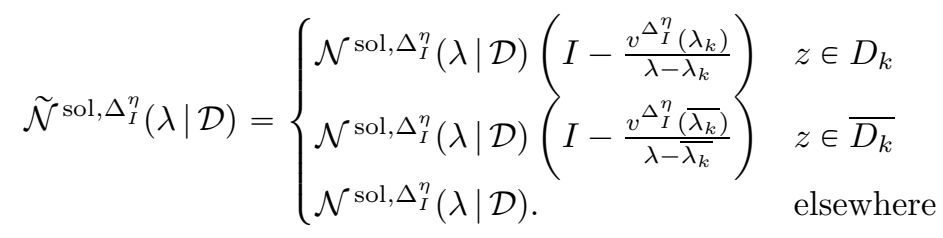

The new unknown $\tilde{\mathcal{N}}^{\text {sol, }, \Delta_{I}^{\eta}}(\lambda \mid \mathcal{D})$ has jumps across each disk boundary

$$
\tilde{\mathcal{N}}_{+}^{\mathrm{sol}, \Delta_{I}^{\eta}}(\lambda \mid \mathcal{D})=\tilde{\mathcal{N}}_{-}^{\mathrm{sol}, \Delta_{I}^{\eta}}(\lambda \mid \mathcal{D}) \widetilde{v}(\lambda)
$$

where $\widetilde{v}(\lambda)$ is given on $\partial D_{k}$ (resp. $\overline{\partial D_{k}}$ ) by the last factor in the first (resp. second) line of (100). By virtue of (99) these jumps satisfy

$$
\|\widetilde{v}(\lambda)-I\|_{L^{\infty}(\tilde{\Sigma})}=\mathcal{O}\left(e^{-4 \mathrm{~d}_{\Lambda} \nu_{0}|t|}\right), \quad \tilde{\Sigma}=\bigcup_{\lambda_{k} \in \Lambda^{+} \backslash \Lambda(I)}\left(\partial D_{k} \cup \overline{\partial D}_{k}\right) .
$$


Next we observe that $\mathcal{N}^{\text {sol, }, \varnothing}(\lambda \mid \widehat{\mathcal{D}})$ has the same poles as $\tilde{\mathcal{N}}_{+}^{\text {sol, } \Delta_{I}^{\eta}}(\lambda \mid \mathcal{D})$ with exactly the same residue conditions. A simple calculation shows that the quantity

$$
e(\lambda)=\tilde{\mathcal{N}}_{+}^{\text {sol }, \Delta_{I}^{\eta}}(\lambda \mid \mathcal{D})\left[\mathcal{N}^{\text {sol }, \varnothing}(\lambda \mid \widehat{\mathcal{D}})\right]^{-1}
$$

has no poles with jump $e_{+}(\lambda)=e_{-}(\lambda) v_{e}(\lambda)$. Here

$$
v_{e}(\lambda)=\left[\mathcal{N}^{\mathrm{sol}, \varnothing}(\lambda \mid \widehat{\mathcal{D}})\right] \widetilde{v}(\lambda)\left[\mathcal{N}^{\mathrm{sol}, \varnothing}(\lambda \mid \widehat{\mathcal{D}})\right]^{-1}, \quad \lambda \in \widetilde{\Sigma}
$$

satisfies an estimate identical to (101) by Lemma B.3 applied to $\mathcal{N}$ sol, $\varnothing(\lambda \mid \widehat{\mathcal{D}})$. Using the theory of small norm RHPs [31, 29], one shows that $e$ exists and that $e(\lambda)=I+\mathcal{O}\left(e^{-4 \mathrm{~d}_{\Lambda} \nu_{0}|t|}\right)$ for all sufficiently large $|t|$. The result then follows from (100), (102) and (97).

\section{REFERENCES}

[1] Borghese, M., Jenkins, R., McLaughlin, K. T.-R. Long-time asymptotic behavior of the focusing nonlinear Schrödinger equation. Annales Inst. H. Poincaré, Analyse nonlinéaire, https://doi.org/10.1016/j.anihpc.2017.08.006.

[2] Baik, J., Kriecherbauer, T., McLaughlin, K., Miller, P. Discrete orthogonal polynomials. Vol. 164. Annals of Mathematics Studies. Asymptotics and applications. Princeton University Press, Princeton, NJ, 2007.

[3] Colin, M., Ohta, M. Stability of solitary waves for derivative nonlinear Schrödinger equation, Ann. Inst. H. Poincar; Analyse Non Linéaire, 23 (2006), 753-764.

[4] Cuccagna, S., Jenkins, R. On the asymptotic stability of N-soliton solutions of the defocusing nonlinear Schrdinger equation. Comm. Math. Phys. 343 (2016), no. 3, 921-969.

[5] Deift, P., Kamvissis, S., Kriecherbauer, T., Zhou, X. The Toda rarefaction problem. Comm. Pure. Appl. Math., 49, no.1 (1996), 35-83.

[6] Deift, P. A.; Its, A. R.; Zhou, X. Long-time asymptotics for integrable nonlinear wave equations. Important developments in soliton theory, 181-204, Springer Ser. Nonlinear Dynam., Springer, Berlin, 1993.

[7] Deift, P., Zhou, X. A steepest descent method for oscillatory Riemann-Hilbert problems. Asymptotics for the MKdV equation. Ann. of Math. (2) 137, no. 2 (1993), 295-368.

[8] Deift, P., Zhou, X. Long-time asymptotics for solutions of the NLS equation with initial data in a weighted Sobolev space. Dedicated to the memory of Jürgen K. Moser. Comm. Pure Appl. Math. 56 (2003), 1029-1077.

[9] Dieng, M., McLaughlin, K D.-T. Long-time Asymptotics for the NLS equation via dbar methods. Preprint, arXiv:0805.2807, 2008.

[10] NIST Digital Library of Mathematical Functions. http://dlmf.nist.gov/, Release 1.0.11 of 2016-06-08. Online companion to [26].

[11] Duyckaerts, T., Kenig, C., F. Merle, F. Classification of radial solutions of the focusing, energy- critical wave equation, Cambridge J. of Math., 1, no. 1 (2013), 75-144.

[12] Duyckaerts, T., Jia, H., Kenig, C., Merle, F. Soliton resolution along a sequence of times for the focusing energy critical wave equation. Preprint, arXiv:1601.01871v2, 2016.

[13] Duyckaerts, T., Kenig, C., Merle, F. Profiles for bounded solutions of dispersive equations, with applications to energy-critical wave and Schrödinger equations, Commun. Pure Appl. Anal., 14, no. 4 (2015), 1275-1326.

[14] Eckhaus, W., P. Schuur, P. The emergence of solitons of the Korteweg-de Vries equation from arbitrary initial conditions, Math. Methods Appl. Sci., 5 , no. 1 (1983), 97-116.

[15] Its, A. R. Asymptotic behavior of the solutions to the nonlinear Schrödinger equation, and isomonodromic deformations of systems of linear differential equations. Dokl. Akad. Nauk SSSR 261, no.1 (1981), 14-18.

[16] Jenkins, R., Liu, J., Perry, P., and Sulem. C. Global well-posedness and soliton resolution for the derivative nonlinear Schrödinger equation. Preprint, arXiv:1706.06252, 2017.

[17] Jenkins, R., Liu, J., Perry, P., and Sulem. C. Global well-posedness for the derivative nonlinear Schrödinger equation. Preprint, arXiv:1710.03810, submitted to Comm. P. D. E. 
[18] Kaup, D. J., Newell, A. C. (1978). An exact solution for a derivative nonlinear Schrödinger equation. J. Math. Phys., 19 (1978), 798-801.

[19] Kitaev, A. V., Vartanian, A. H. Leading-order temporal asymptotics of the modified nonlinear Schrödinger equation: solitonless sector. Inverse Problems 13, no. 5 (1997), 1311-1339.

[20] Kitaev, A. V., Vartanian, A. H. Asymptotics of solutions to the modified nonlinear Schrödinger equation: solitons on a nonvanishing continuous background. SIAM J. Math. Anal. 30, no. 4 (1999), 787-832.

[21] Liu, J. Global well-posedness for the derivative nonlinear Schrödinger equation through inverse scattering. Thesis, University of Kentucky, 2017. https://uknowledge.uky.edu/math_etds/50.

[22] Liu, J., Perry, P., Sulem, C. Global existence for the derivative nonlinear Schrödinger equation by the method of inverse scattering. Comm. Part. Diff. Eqs 41 no. 11 (2016), 1692-1760.

[23] Liu, J., Perry, P., Sulem, C. Long-time behavior of solutions to the derivative nonlinear Schrdinger equation for soliton-free initial data. Ann. Inst. H. Poincar Anal. Non Linéaire 35 (2018), no. 1, 217-265. https://doi.org/10.1016/j.anihpc.2017.04.002.

[24] McLaughlin, K. T.-R.; Miller, P. D. The $\bar{\partial}$ steepest descent method and the asymptotic behavior of polynomials orthogonal on the unit circle with fixed and exponentially varying nonanalytic weights. IMRP Int. Math. Res. Pap. (2006), Art. ID 48673, 1-77.

[25] Muscalu, Camil; Tao, Terence; Thiele, Christoph. A Carleson theorem for a Cantor group model of the scattering transform. Nonlinearity 16 (2003), no. 1, 219-246.

[26] Olver, F., Lozier, D., Boisvert, R., and Clark, C., editors. NIST Handbook of Mathematical Functions. Cambridge University Press, New York, NY, 2010. Print companion to [10].

[27] Schuur, P. Asymptotic analysis of soliton problems. An inverse scattering approach. Lecture Notes in Mathematics, 1232, Springer-Verlag, Berlin, 1986.

[28] Tao, T. A global compact attractor for high-dimensional defocusing nonlinear Schrödinger equations with potential. Dyn. Part. Diff. Eqs., 5 no. 2 (2008), 101-116.

[29] Trogdon, T., Olver, S. Riemann-Hilbert problems, their numerical solution, and the computation of nonlinear special functions. Society for Industrial and Applied Mathematics (SIAM), Philadelphia, PA, 2016.

[30] Zakharov, V.E., Shabat, A.B. Exact theory of two-dimensional self-focusing and onedimensional self-modulation of waves in nonlinear media. Soviet Physics JETP, 34, no. 1 (1972), 62-69.

[31] Zhou, X. The Riemann-Hilbert problem and inverse scattering. SIAM J. Math. Anal. 20, no. 4 (1989), 966-986.

[32] Zhou, X. Direct and inverse scattering transforms with arbitrary spectral singularities. Comm. Pure Appl. Math. 42 (1989), no. 7, 895-938.

(Jenkins) Department of Mathematics, University of Arizona, Tucson, AZ 85721-0089 USA

(Liu) Department of Mathematics, University of Toronto, Toronto, Ontario M5S 2E4, CANADA

(Perry) Department of Mathematics, University of Kentucky, Lexington, Kentucky 40506-0027

(Sulem) Department of Mathematics, University of Toronto, Toronto, Ontario M5S 2E4, CANADA 